University of Louisville

ThinkIR: The University of Louisville's Institutional Repository

$12-2019$

\title{
A recombinant cholera toxin b subunit variant (CTB-KDEL) exhibits unique colon mucosal healing effects that have therapeutic implications for inflammatory bowel disease.
}

Joshua Mark Royal

University of Louisville

Follow this and additional works at: https://ir.library.louisville.edu/etd

Part of the Pharmaceutics and Drug Design Commons, and the Translational Medical Research Commons

\section{Recommended Citation}

Royal, Joshua Mark, "A recombinant cholera toxin b subunit variant (CTB-KDEL) exhibits unique colon mucosal healing effects that have therapeutic implications for inflammatory bowel disease." (2019). Electronic Theses and Dissertations. Paper 3317.

https://doi.org/10.18297/etd/3317

This Doctoral Dissertation is brought to you for free and open access by ThinkIR: The University of Louisville's Institutional Repository. It has been accepted for inclusion in Electronic Theses and Dissertations by an authorized administrator of ThinkIR: The University of Louisville's Institutional Repository. This title appears here courtesy of the author, who has retained all other copyrights. For more information, please contact thinkir@louisville.edu. 


\title{
A RECOMBINANT CHOLERA TOXIN B SUBUNIT VARIANT (CTB-KDEL) \\ EXHIBITS UNIQUE COLON MUCOSAL HEALING EFFECTS THAT HAVE \\ THERAPEUTIC IMPLICATIONS FOR INFLAMMATORY BOWEL DISEASE
}

\author{
By \\ Joshua Mark Royal \\ B.S., Western Kentucky University, 2013 \\ M.S., University of Louisville, 2018

\begin{abstract}
A Dissertation
Submitted to the Faculty of the

School of Medicine of the University of Louisville

In Partial Fulfillment of the Requirements for the Degree of
\end{abstract}

Doctor of Philosophy in Pharmacology and Toxicology

Department of Pharmacology and Toxicology

University of Louisville

Louisville, KY

December 2019 
Copyright 2019 by Joshua Mark Royal

All rights reserved 

A RECOMBINANT CHOLERA TOXIN B SUBUNIT VARIANT (CTB-KDEL) EXHIBITS UNIQUE COLON MUCOSAL HEALING EFFECTS THAT HAVE THERAPEUTIC IMPLICATIONS FOR INFLAMMATORY BOWEL DISEASE

\author{
By \\ Joshua M. Royal \\ B.S. Western Kentucky University, 2013 \\ M.S., University of Louisville, 2018
}

A Dissertation Approved on

November 22, 2019

By the following Dissertation Committee:

Nobuyuki Matoba, Ph.D.

Brian Ceresa, Ph.D.

Leah Siskind, Ph.D.

Kavitha Yaddanapudi, Ph.D.

Chi Li, Ph.D. 


\title{
DEDICATION
}

This dissertation is dedicated to my wife

\author{
Ellie F. Royal
}

And my Parents

Mr. Timothy Royal and Mrs. Sheilia Royal,

Who have supported me at home throughout this process

And

Nobuyuki Matoba, Ph.D.

Whose mentorship exemplifies selflessness, wisdom, and a dedication to education.

七転び八起き 


\section{ACKNOWLEDGEMENTS}

I would like to thank all of my committee members, Nobuyuki Matoba, Brian Ceresa, Leah Siskind, Chi Lee and Kavitha Yaddanapudi, who have provided pivotal guidance to my development as a scientist. All of these individuals have influenced me to pursue a life of continued education and dedication to science. I am extremely grateful for the wisdom, guidance, and scientific opportunities provided over the years by my collaborators; Dr. Youngjun Oh, Dr. Susan Galandiuk, Dr. Nemencio Ronquillo, Dr. Michael Grey, and Dr. Wayne Lencer. I am exceptionally appreciative to my Mentor, Dr. Nobuyuki Matoba, for guiding me through my adolescence as a scientist and always pushing me to improve. Also, I would like to thank my wife, Ellie Royal, who from the beginning supported me and encouraged me throughout this process and to press on when times were tough, she deserves more thanks then I could possibly express here. Lastly, I am extremely grateful of the love and encouragement I have receive from my parents and brothers throughout my time here at the University of Louisville. 


\begin{abstract}
A RECOMBINANT CHOLERA TOXIN B SUBUNIT VARIANT (CTB-KDEL) EXHIBITS UNIQUE COLON MUCOSAL HEALING EFFECTS THAT HAVE THERAPEUTIC IMPLICATIONS FOR INFLAMMATORY BOWEL DISEASE Joshua M. Royal
\end{abstract}

November 22, 2019

This dissertation uncovers the mechanism and explores the utility of a recombinant cholera toxin B subunit (CTB) variant containing a KDEL endoplasmic reticulum (ER) retention motif (CTB-KDEL) as a drug candidate for the treatment of inflammatory bowel disease (IBD). CTB is a mucosal immunomodulatory protein that induces robust mucosal and systemic antibody responses. This well-known biological activity has been exploited in cholera prevention (as a component of Dukoral ${ }^{\circledR}$ vaccine) and vaccine development for decades. On the other hand, several studies have investigated CTB's immunotherapeutic potential in the treatment of inflammatory diseases such as Crohn's disease and asthma. Previously, we found that the plant-made variant of CTB (CTB-KDEL) could induce colon epithelial wound healing in mouse colitis models. Herein, Chapter 1 discusses the diverse therapeutic applications of CTB and CTB variants. The methodology of the studies presented herein are described in 
Chapter2. Chapter 3 reveals CTB-KDEL induces colon epithelial wound healing in colitis via the activation of an unfolded protein response (UPR) in colon epithelial cells. CTB-KDEL's capacity to induce a UPR and epithelial restitution/wound healing was corroborated in a dextran sodium sulfate (DSS)-induced acute colitis mouse model. Chapter 4 describes the feasibility of repeated oral administration of CTB-KDEL for the treatment of chronic colitis. This chapter addresses two key concerns with the development of CTB-KDEL for the treatment of IBD. First, can CTB-KDEL alleviate chronic inflammatory conditions of IBD, specifically in an ulcerative colitis model, and second, what are the consequences of CTB-KDEL induced immunogenicity on its efficacy. We found that weekly (QW) oral administration of CTB-KDEL dosed before or dosed after the onset of chronic conditions with QW and every three-day dosing (Q3D) could significantly reduce disease severity. The results indicate that the animals continued to respond to the medication over time and did not lose its efficacy with repeated dosing. Furthermore, the positive results in the delayed dosing group, mice dosed after the onset of chronic conditions, indicate that CTB-KDEL can alleviate chronic inflammation and reverse DSS-induced colonic damage. The second part of Chapter 4 addresses the consequences of CTB-KDEL induced immunogenicity on its therapeutic effect. As CTB-KDEL is a derivative of the cholera vaccine antigen CTB, it is of critical importance to assess the consequences of an immune response to CTBKDEL. Pre-dosing mice with CTB-KDEL indeed elicited significant levels of anti-CTBKDEL IgA antibodies in the intestine before DSS exposure and throughout the rest of the study, however, those ADAs did not alter the therapeutic effect of CTB-KDEL against DSS colitis. Conversely, the immunogenicity of CTB could be beneficial for colitis treatment, since induction of $\operatorname{IgA}$ is associated with an anti-inflammatory response and 
IgA plays an important role in maintaining mucosal homeostasis. Thus, we performed the same acute DSS colitis model using $\mathrm{C} 3 \mathrm{H}$ mice since this strain cannot produce ADAs against CTB. Subsequently, we found that indeed CTB-KDEL could mitigate DSS colitis without inducing an antibody response. These results suggest that CTB-KDEL's immunogenicity is not beneficial nor detrimental to its therapeutic effect. Lastly, in Chapter 5, we describe CTB-KDEL's therapeutic effects on IBD patient colon explants. To simulate CTB-KDEL's therapeutic potential in humans, its effects on epithelial repair responses and histological changes were evaluated in colon tissues obtained from IBD patients with various disease history and biological backgrounds. The results demonstrate CTB-KDEL's efficacy in this translational model, as manifested by the induction of $T G F B$ gene expression, upregulation of wound healing pathway genes and presence of viable crypts in the mucosa. Chapter 6 summarizes the effects of CTB-KDEL revealed in this dissertation and discusses future directions.

In summary, CTB-KDEL exhibits unique colon mucosal would healing effects mediated by its colocalization to the ER and subsequent activation of IRE1/XBP1 signaling in colon epithelial cells. Repeated oral administration of a CTB-KDEL effectively mitigates chronic DSS-colitis despite a robust immunogenic response. Additionally, CTB-KDEL induces wound healing responses in IBD patient colon tissues. These results presented herein provide implications for the unique therapeutic potential of CTB-KDEL that may address a significant unmet need in IBD treatment. 


\section{TABLE OF CONTENTS}

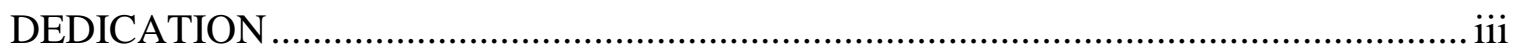

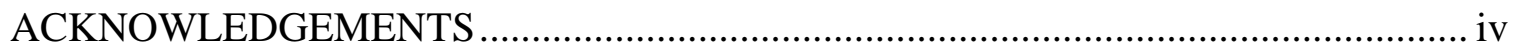

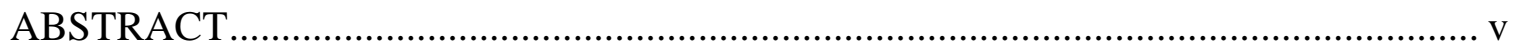

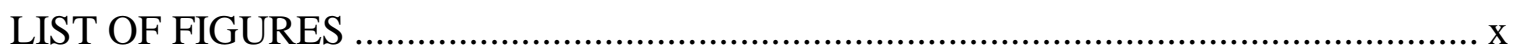

CHAPTER 1: THERAPEUTIC POTENTIAL OF CHOLERA TOXIN B SUBUNIT FOR THE TREATMENT OF INFLAMMATORY DISEASES OF THE MUCOSA .............. 1

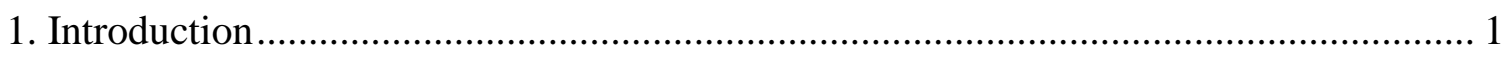

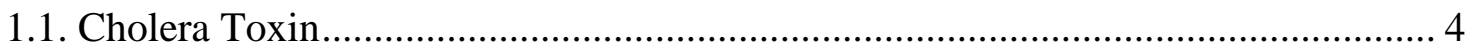

1.2. Cholera Toxin Structure and Mechanism in Gut Epithelial Cells......................... 6

1.3. Mode of Action: CTB Directly Modulates Immune and Epithelial Cells................ 8

1.3.1. CTB Directly Impacts Immune Cells ........................................................ 9

1.3.2. A Plant-Made CTB variant (CTB-KDEL) Directly Modulates Epithelial Cells 13

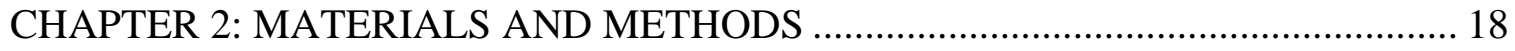

CHAPTER 3: A MODIFIED CHOLERA TOXIN B SUBUNIT CONTAINING AN ER RETENTION MOTIF ENHANCES COLON EPITHELIAL REPAIR VIA AN UNFOLDED PROTEIN RESPONSE .................................................................... 28

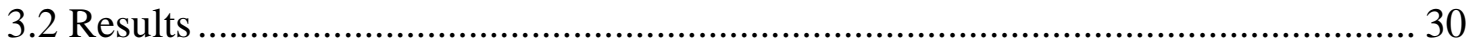

3.2.1 The C-Terminal KDEL Sequence is Essential for the Colon Epithelial Repair

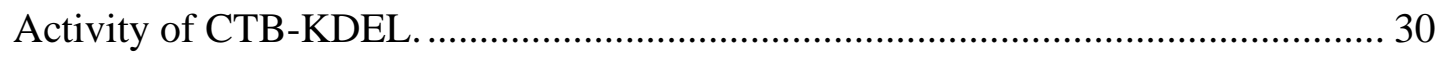

3.2.2 CTB-KDEL Induces an Unfolded Protein Response and IRE1-XBP1 Signaling

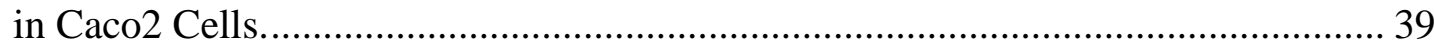

3.2.3 CTB-KDEL Induces A UPR in Primary Colon Epithelial Cells and Facilitates Colonic Wound Healing After DSS-Induced Acute Colitis in Mice......................... 44

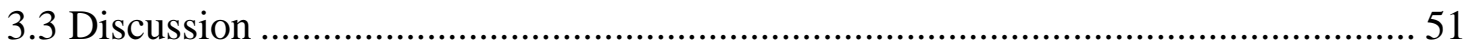


CHAPTER 4: REPEATED ORAL ADMINISTRATION OF A KDEL-TAGGED RECOMBINANT CHOLERA TOXIN B SUBUNIT EFFECTIVELY MITIGATES DSS COLITIS DESPITE A ROBUST IMMUNOGENIC RESPONSE .................................. 56

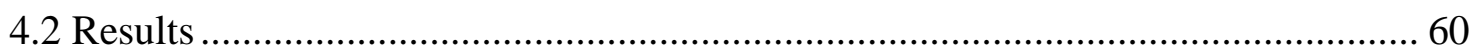

4.2.1 CTB-KDEL's Therapeutic Effects in a Mouse Colitis Model Exposed to

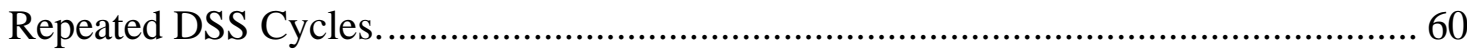

4.2.2 The Impact of CTB-KDEL's Immunogenicity on its Therapeutic Effect in Acute

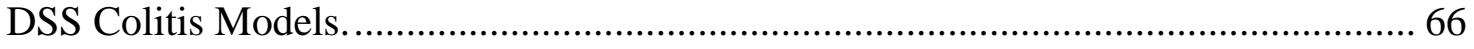

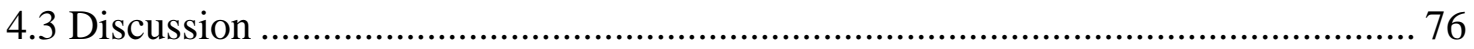

CHAPTER 5: CTB-KDEL INDUCES A WOUND HEALING RESPONSE IN HUMAN

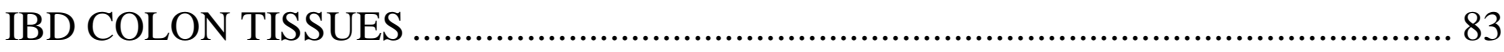

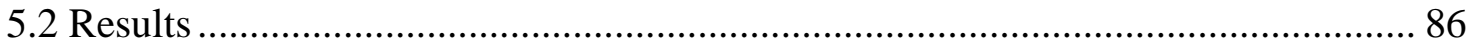

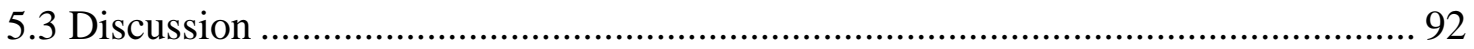

CHAPTER 6: SUMMARY AND IMPLICATIONS FOR FUTURE DIRECTIONS..... 94

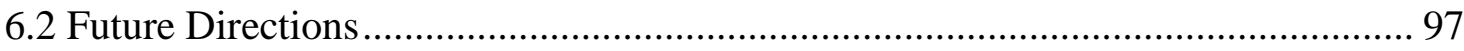

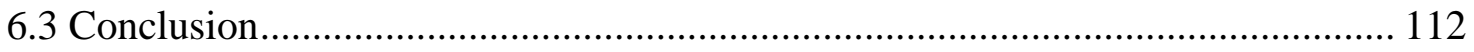

REFERENCES ............................................................................................. 113

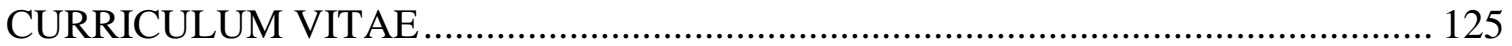




\section{LIST OF FIGURES}

Figure 1. Summary of mechanisms involved in cholera toxin homopentameric B-subunit (CTB)'s inflammatory disease intervention. 13

Figure 2. CTB-KDEL, but not CTB, enhances cell migration and TGF $\beta$ levels in a human colon epithelial cell wound healing model.. 32

Figure 3. Escherichia coli produced CTB-KDEL enhances cell migration. ................... 34

Figure 4. Stability of CTB and CTB-KDEL in Caco2 cells. ...................................... 35

Figure 5. CTB-KDEL binds to the KDEL receptor (KDELR).................................. 38

Figure 6. CTB-KDEL induces the unfolded protein response (UPR). .......................... 40

Figure 7. CTB-KDEL's epithelial wound healing activity is mediated through IRE1-

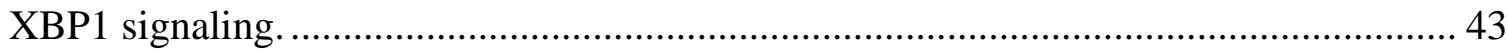

Figure 8. Immunoblot analysis of UPR-related proteins. ....................................... 45

Figure 9. Effects of orally administered CTB or CTB-KDEL in an acute DSS-colitis

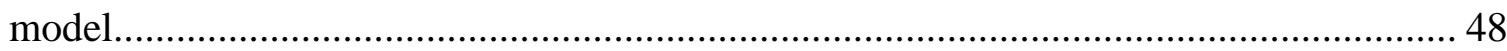

Figure 10. CTB-KDEL and CTB effects on mouse colon histological alterations induce

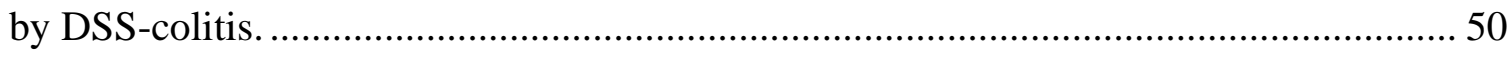

Figure 11 Dextran sodium sulfate (DSS) chronic colitis study design........................ 60

Figure 12. CTB-KDEL mitigates DSS-induced injury and inflammation in a DSS chronic

colitis study 
Figure 13. Histological findings from the DSS chronic colitis model.......................... 66

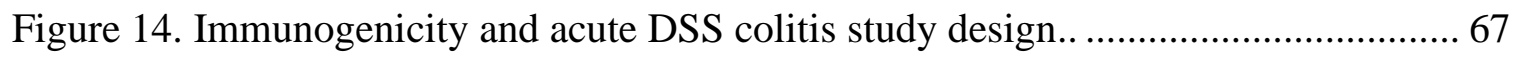

Figure 15. The impact of a pre-existing immune response to CTB-KDEL on its therapeutic efficacy against DSS acute colitis in C57BL/6 mice ................................ 70 Figure 16. The impact of CTB-KDEL predosing on its therapeutic efficacy against DSS

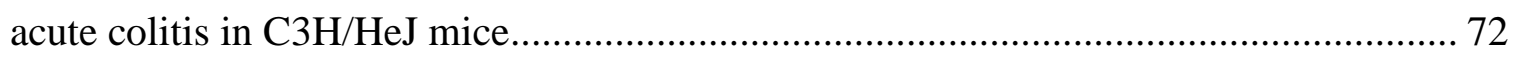
Figure 17. Analysis of CTB-KDEL's therapeutic efficacy against DSS acute colitis in

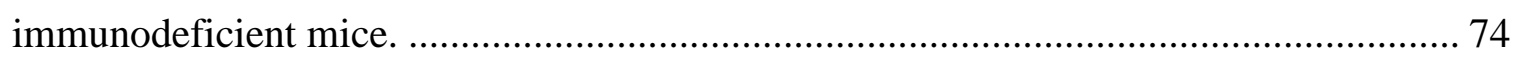
Figure 18. Effects of CTB-KDEL and CTB in human colon tissue isolated from a 57-

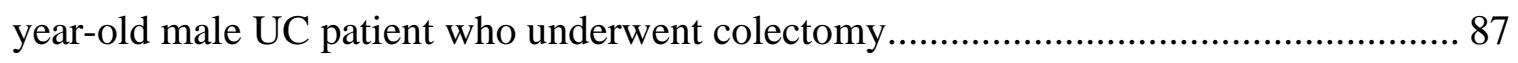
Figure 19. Effects of CTB-KDEL and CTB in human colon tissue from IBD patients who

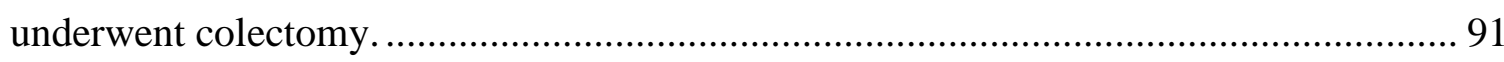

Figure 20. Characterization of G33D-CTB-KDEL ............................................ 101 Figure 21. G33D-CTB-KDEL loosely binds to Caco2 cells and localizes with the KDEL

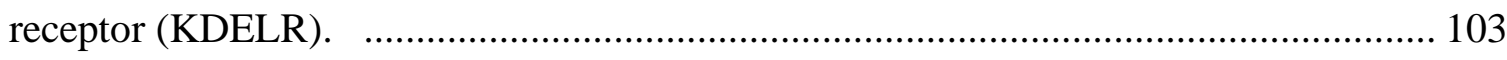




\section{CHAPTER 1: THERAPEUTIC POTENTIAL OF CHOLERA TOXIN B SUBUNIT FOR THE TREATMENT OF INFLAMMATORY DISEASES OF THE MUCOSA ${ }^{1}$}

\section{Introduction}

UC is a class of inflammatory bowel diseases (IBD), along with Crohn's disease (CD), that affects 286 per 100,000 persons ( $0.3 \%$ of total population) in the USA alone [1]. Although the etiology of UC remains elusive, genetic and environmental factors appear to trigger dysregulated mucosal immune responses, leading to the onset and progression of chronic inflammation, disrupted intestinal barrier function and epithelial damage in the colon [2]. There is no curative therapy available for UC; conventional treatment strategies aim to blunt the inflammatory response and establish remission using corticosteroids, aminosalicylates and immunosuppressive agents, but these agents have limited efficacy or severe adverse reactions, often requiring additional treatment or surgical resection of the colon and rectum [3]. The recent advent of anti-TNF $\alpha$ agents, however, has greatly improved the clinical outcome of IBD patients and revealed that mucosal healing provides an important endpoint in IBD treatment, as it is closely associated with sustained clinical remission, improved quality of life and fewer surgical operations and cancer incidence [4-9]. In a study evaluating 5-year treatment outcomes of

\footnotetext{
${ }^{1}$ Modified from: Toxins (Basel). 2017 Nov 23;9(12). pii: E379. doi: 10.3390/toxins9120379.
} 
patients with newly diagnosed UC receiving corticosteroid therapy, the lack of mucosal healing was found to be the only factor associated with a more aggressive disease outcome requiring immunosuppression therapy, hospitalization or colectomy [10]. In two randomized, double-blind, placebo-controlled studies (ACT1 and 2), patients treated with the anti-TNF $\alpha$ infliximab who exhibited mucosal healing at week 8 showed a $\sim 5$-fold higher rate of clinical remission at week 30 than those without mucosal healing [11]. In a study of 513 Norwegian patients diagnosed with UC, mucosal healing was significantly associated with a low risk of future colectomy at a 5-year follow-up [12]. These clinical data have led to a general consensus that mucosal healing is the most important treatment goal in UC $[13,14]$. However, a major problem is that current therapeutic options can achieve mucosal healing in only up to half of patients $[15,16]$; in ACT1 trial, $45-47 \%$ of those who received infliximab achieved mucosal healing, and similar results were observed in ACT2 trial [11]. Similarly, in a Phase III clinical trial of the anti-TNF $\alpha$ Adalimumab, $46.7 \%$ of patients who received the antibody showed mucosal healing [17]. The relatively low rates of mucosal healing is likely because the currently available UC drugs are, by and large, anti-inflammatory agents that do not specifically stimulate a tissue healing process. Mucosal healing is a complex and dynamic biological process involving spatial and temporal network of a number of mediators and cell types including epithelial, immune and stromal cells $[5,18]$. Thus, mere inhibition of inflammatory responses does not necessarily facilitate mucosal healing. Development of a new drug specifically targeting mucosal healing will fill the gap in the current UC treatment paradigm.

A clear definition of mucosal healing has yet to be determined $[5,18]$. It has been inferred that mucosal healing involves the coordination of IECs, goblet cells, and Paneth 
cells for healing of intestinal barrier function [5]. Additionally, intestinal epithelial wound repair is especially critical for mucosal healing in UC patients, because UC's pathology is limited to the mucosa unlike CD that manifest as transmural disease [5], epithelial would healing has particularly significant therapeutic implications in UC [19]. Wound repair has been extensively studied in non-mucosal tissues such as the skin, but the same mechanism seems to be applicable to mucosae [18]; it involves three main overlapping phases, inflammation, proliferation and maturation. Each phase relies on the appropriate levels of cytokines and growth factors in the wound environment [20, 21]. Among these, TGF $\beta$ plays critical roles across all three healing phases; in the inflammation phase, TGF $\beta$ induces the recruitment of neutrophils and macrophages to "clean up" the wound environment and subsequently regulates immune cells to resolve inflammation. In the proliferation phase, TGF $\beta$ promotes angiogenesis, fibroblast proliferation and production of extracellular matrix (ECM) components, which leads to granulation tissue formation and wound contraction. In the maturation phase, TGF $\beta$ contributes to ECM remodeling by regulating the expression of various enzymes including matrix metalloproteinases (MMPs) and tissue inhibitors of MMPs (TIMPs) [5, 18, 20-22]. Importantly, TGF $\beta$ can be a double-edged sword in mucosal remodeling; while TGF $\beta$ has beneficial effects during the early stage of wound healing, it can promote scar formation and fibrosis at the late stage [20, 23]. In addition, although TGF $\beta$ functions as a suppressor of epithelial cell tumorigenesis at an early stage of tumor development, its expression is correlated with tumor progression and poor prognosis at late stages [23]. Thus, a tight control of TGF $\beta$ signaling, when targeted to induce mucosal healing in UC, is critical, because UC poses an increased risk of developing colitisassociated colorectal cancer (CAC) $[24,25]$. Given that mucosal inflammation of IBD 
reflects composite conditions including both nascent and established inflammations [26]. It is likely that TGF $\beta$-mediated wound repair remains to play a significant role at least in some parts of UC colon mucosa.

As discussed above, the development of a new drug specifically targeting mucosal healing could fill a gap in the current UC treatment paradigm. Unfortunately, there are no drugs specifically targeting mucosal healing currently available or in clinical development. Therefore, the goal of the studies presented herein is to uncover a first-inclass therapeutic candidate for UC that can effectively treat intestinal damage and induce mucosal healing without suppressing systemic immune functions, a significant unmet need in current UC treatment paradigm. This chapter describes the utility of a recombinant cholera toxin B subunit (CTB) variant as a drug candidate for the treatment of inflammatory diseases of the mucosa.

\subsection{Cholera Toxin}

Vibrio cholerae is a gram-negative bacterium that can colonize the gastrointestinal tract and cause life-threatening disease termed Cholera. Approximately 2.9 million cases and 95,000 deaths occur each year around the world due to Vibrio cholerae infection. While the infection is often mild or without symptoms, $10 \%$ of infected persons will have severe disease that cause rapid loss of body fluids caused by profuse diarrhea and vomiting. If left untreated, death can occur within hours [27]. The principal virulence factor of $V$. cholerae is cholera toxin (CT), which consists of a catalytic A-subunit and a non-toxic homopentameric B-subunit (CTB) [28-30]. CTB binds cells through GM1 ganglioside receptors, which then mediates toxin entry into the cell. It has been previously shown that CTB can induce strong biological activities that 
can enhance or suppress immune effects under normal and various immunopathological conditions without the toxicity associated with the CTA subunit [31]. Consequently, CTB has been widely studied as a mucosal immunomodulatory agent.

In its most well-known immunostimulatory effects, CTB is used in the vaccine Dukoral $^{\circledR}$. Dukoral ${ }^{\circledR}$ is a WHO pre-qualified oral cholera vaccine, which contains heatkilled whole cell $V$. cholerae and recombinant CTB (CTB; Table 1). Dukoral ${ }^{\circledR}$ stimulates the production of both antibacterial and antitoxin antibodies, including secretory immunoglobulin A (S-IgA) produced locally in the intestines [32]. CTB itself can induce potent mucosal and systemic antibody response upon mucosal administration in humans [33-35], which is largely due to the broad distribution of GM1 ganglioside on various cell types such as epithelial cells, macrophages, dendritic cells (DCs), B cells, T cells, and neurons [36-39]. Furthermore, the presence of GM1 ganglioside on the luminal surface of intestinal epithelial cells (IECs) and antigen presenting cells (APCs) in the gut seems to be essential for CTB's strong mucosal immunostimulatory effects associated with MHC class II expression and local antigen enrichment [40]. Due to CTB's strong mucosal immunomodulatory effects and the use of CTB in the vaccine Dukoral, the production of the protein in various recombinant production platforms has been widespread. These include eukaryotes yeast cells to multicellular organisms such as plants and silkworms, as well as, prokaryotic cells such as genetically modified V. cholerae, E. coli, Bacillus and Lactobacillus (reviewed in [31]). In cell culture systems, recombinant CTB is produced in fermenters and bioreactors [31]. Alternatively, in plant expression systems, CTB is expressed in whole plants grown in controlled growth rooms or greenhouses. Previously, our lab has produced recombinant CTB in tobacco plants (Nicotiana benthamiana) using a plant virus vector overexpression system (CTB-KDEL; plant-made CTB; Table 1), with 
the aim to economically mass-produce the vaccine antigen to facilitate global cholera vaccination [41-43]. CTB-KDEL was generated as a result of this endeavor; we genetically modified CTB to add an ER retention signal to the C-terminus, which was critical to mitigate ER stress/tissue necrosis upon overexpression and achieve high-level accumulation in leaf tissue $[41,42]$. Our analyses demonstrated that CTB-KDEL is virtually identical to original CTB in regards to GM1-ganglioside binding affinity, molecular stability and vaccine efficacy to induce anti-toxin IgG and IgA antibodies upon oral immunization in mice [41]. Subsequently, we established a facile and scalable CTBKDEL production process, which allows us to obtain $400 \mathrm{mg}$ of the purified protein from $1 \mathrm{~kg}$ of leaf biomass (corresponding to 400 doses of Dukoral vaccine), with quality that can meet pharmaceutical standards [44].

In addition to CTB's immunostimulatory effects, CTB stimulates specific immunosuppressive effects against autoimmune disorders, excess inflammation, and allergic reactions [31, 45-49]. We have recently shown that oral administration of CTBKDEL mitigates colitis in chemically-induced acute and chronic colitis mouse models [50]. Although the underlying mechanisms are not well understood, recent studies have shed some light on these immunosuppressive effects induced by CTB. In this chapter, I summarize possible mechanisms behind CTB's anti-inflammatory activity and discuss how the protein could impact mucosal inflammatory disease treatment.

\subsection{Cholera Toxin Structure and Mechanism in Gut Epithelial Cells}

To reveal the mechanism of CTB-induced biological activity, we must first understand the molecule. CT is classified as an AB5 toxin family, which includes the toxins of Shigella dysenteriae and enterohaemorrhagic Escherichia coli. The toxins are 
usually composed of one A subunit and five B subunits (CTA and CTB, respectively, for CT). CTA consists of an enzymatically active 11-kDa N-terminal chain (CTA1) and a Cterminal chain (CTA2) that connects CTA to the central pore of CTB. CTB has the capacity to translocate the CTA across the plasma membrane, mediated by the binding of GM1 ganglioside, and then escort CTA from the plasma membrane into the endoplasmic reticulum (ER) [51, 52]. The following summarizes CT's retrograde trafficking mechanism.

The five B-subunits form a central cylindrical pore lined by five amphipathic $\alpha$ helices that help form a highly stable homopentamer. The pentamer contains five GM1 binding sites that lie on the outer edge of each B subunit $[28,53]$. Due to an avidity effect from the pentavalent binding capacity, CTB has a very strong affinity $\left(\mathrm{K}_{\mathrm{D}}\right.$ reported to be $5 \mathrm{pM}$ to $1 \mathrm{nM}$ ) to GM1, which is mainly localized in lipid rafts on the plasma membranes of many cell types [36-39]. Once CT is bound to GM1 (up to five gangliosides at once), it is endocytosed by clathrin-dependent and independent mechanisms and trafficked via retrograde transport from the Golgi to the ER [52]. It is also known that CT can undergo transcytosis across epithelial cells from the apical to the basolateral surface. However, regardless of how the toxin enters the cell, CT travels to the trans-Golgi network via early endosomal vesicles, independent of the late endosome pathway. The C-terminus of CTA2 possesses a KDEL ER-retention signal for retrieval of CT from the cis-Golgi apparatus to the ER. Interestingly, the KDEL sequence is not vital for retrograde transport of CT to the ER. Mutations that alter the KDEL sequence on CT inhibit KDEL-dependent ER retrieval and decreased (albeit not completely) CT's toxification [54]. Thus, it is thought that CT's KDEL sequence-although not absolutely essential-improves the ER's retrieval of the dissociated CT from the Golgi apparatus and prolongs the time of 
retention within the ER $[51,54,55]$. Once in the ER, the CTA1-chain is dissociated from CTA2/CTB complex by protein disulfide isomerase (PDI). Subsequently, CTA1 enters the cytosol via the ER-associated degradation pathway and escapes proteasomal degradation $[28,51]$. On the other hand, the fate (and remaining function, if any) of CTA2/CTB after releasing CTA1 in the ER is not well documented. Meanwhile, CTA1 reaches the inner surface of the plasma membrane and catalyzes the ADP ribosylation of Gas, thereby continuously activating adenylate cyclase to produce cAMP. Increased intracellular cAMP impairs sodium uptake and increases chloride outflow, leading to water secretion and diarrhea $[51,56]$.

\subsection{Mode of Action: CTB Directly Modulates Immune and Epithelial Cells}

Although the virulence mechanism and intracellular trafficking of CT has been well studied, the anti-inflammatory mechanisms of CTB are much less studied and understood. After a comprehensive literature review, it seems that there are at least two separate modes of action induced by CTB to modulate inflammatory responses: one that is based on immune cell regulation and another that is epithelial cell-mediated (Figure 1).

In 1994, the immune suppressive effects of CTB were first reported by Sun et al. [57]. This report demonstrated that oral administration of mice with CTB conjugated with antigens (sheep red blood cells, horse red blood cells, and human $\gamma$-globulin) enhanced oral tolerance to the antigens, presumably through efficient presentation of antigens to immune cells in the gut-associated lymphoid tissue and the generation of regulatory cells. In a Commentary to this article, Weiner suggested that CTB could have enhanced tolerance by serving as a "selective mucosal adjuvant" and that this unique activity could be exploited to treat autoimmunity [58]. Subsequently, this seminal finding led to a new 
field of studies in which CTB-antigen conjugates were applied to induce tolerogenic reactions to the conjugated antigens in various immunopathological conditions (i.e., encephalomyelitis, autoimmune diabetes, autoimmune arthritis, uveitis) and IgEmediated allergen hypersensitivity [45, 47-49, 59-69]. Through these studies, it became apparent there are two unique and distinct mechanisms of CTB responsible for the suppression of immunopathological reactions in allergy and autoimmune diseases: (1) to increase antigen uptake and presentation by different APCs through binding to their cellsurface GM1 ganglioside receptors and (2) to induce anti-inflammatory and immunoregulatory activities by directly or indirectly acting on specific immune cells. The latter mechanism points to the possibility that CTB by itself may act as an immunotherapeutic agent; however, only a handful of groups have proven that CTB alone - without co-administration or conjugation of antigens — can induce an antiinflammatory response. Moreover, studies conducted with non-recombinant CTB (nrCTB, prepared by chemically dissociating CTA from CTB; Table 1) can have significantly skewed experimental results due to trace amounts of CT and CTA remaining in nrCTB samples preparations $[31,70,71]$. For example, we have shown that picomolar concentrations ( $<10 \mathrm{ng} / \mathrm{mL}$ ) of CT significantly inhibited lipopolysaccharide (LPS)induced TNF $\alpha$ production in RAW264.7 cells, while recombinant CTB failed to induce such an effect at a concentration as high as $10 \mu \mathrm{g} / \mathrm{mL}$ [31]. Thus, the use of recombinant CTB is required to evaluate the effects unique to CTB.

\subsubsection{CTB Directly Impacts Immune Cells}

With regards to CTB's immune cell regulation, Kim et al. demonstrated in murine spleen B cells that CTB dose-dependently increased IgA secretion and inhibited B cell 
growth [72]. In the presence of IL-2, CTB significantly increased IgA isotype switching in LPS-activated B cells. These effects were reversed by the addition of an anti-TGF $\beta$ or soluble TGF $\beta 1$ receptor, which markedly inhibited CTB-stimulated IgA response. Further analysis in the same report revealed that CTB stimulated IgA2 B cells, upregulated TGF $\beta 1$ mRNA expression, and increased bioactive TGF $\beta 1$ levels, which is known to induce IgA isotype switching [72]. Thus, CTB stimulated a TGF $\beta$-mediated IgA response that was dependent on IL-2 as a cofactor. These findings have contributed to our understandings of how CTB stimulates B cell IgA production, and potentially oral tolerance as well (see below).

It is known that $\operatorname{IgA}$ antibodies help maintain mucosal homeostasis and play a role in immune protection $[73,74]$. Thus, it seems possible that CTB administration could provide therapeutic effects in mucosal autoimmune disorders via IgA induction. For example, in an experimental mouse model of asthma, nrCTB suppressed the ability of DCs to prime for Th2 responses to inhaled allergen via an IgA-dependent manner [75]. In this study, co-administration of ovalbumin (OVA) and $\mathrm{nrCTB}$ suppressed classical features of asthma, including airway eosinophilia, Th2 cytokine synthesis, and bronchial hyperactivity in mice that were pre-sensitized with OVA-stimulated DCs in the lung. Furthermore, nrCTB treatment enhanced DCs' potential to induce Treg cells in vitro; however, these Treg cells did not provide protection when transferred into the airways of naïve mice that received OVA challenge. In contrast, the transfer of B cells from OVA+CTB-DCs-immunized mice to OVA-sensitized naïve mice significantly reduced eosinophilia and lymphocytosis. It was also found that nrCTB caused a TGF $\beta$-dependent increase in antigen-specific IgA in the airway luminal secretion, and this was attributed to nrCTB's efficacy against the experimental asthma as the therapeutic effects were 
abrogated in mice lacking luminal $\operatorname{Ig} A$ transporter (polymeric Ig receptor), which is necessary for the transport of dimeric IgA across the epithelium into the luminal mucosa [76].

Meanwhile, IgA may not be the sole factor contributing to CTB's ability to mitigate inflammatory diseases in the mucosa. For example, in the 2,4,6-trinitrobenzene sulfonic acid (TNBS)-induced mouse model of Crohn's disease, daily oral administration over a four-day period of $100 \mu \mathrm{g}$ CTB after the onset of TNBS-colitis immediately resolved weight loss and reduced inflammation [70]. In this case, the timing of mucosal restitution in regard to CTB administration did not likely result in IgA production. In a similar TNBS-colitis study, CTB administration reduced IL-12 and IFN $\gamma$ secretion, inhibited STAT-4 and STAT-1 activation, and downregulated T-bet expression, indicating that CTB inhibited mucosal Th1 cell signaling [77]. Moreover, these results were confirmed in a small multicenter, open-label, and nonrandomized clinical trial in which 15 patients with active CD received three oral doses of $5 \mathrm{mg}$ CTB per-week over 2 weeks (six doses total) and were examined 2, 4, 6, and 10 weeks after the start of the study. Of the 12 patients who finished the study per protocol, seven responded to treatment and five were in remission by week six and maintained remission through week 10 as defined by a CD activity index score $\leq 150$ [78]. Of note, side effects seen in $33 \%$ of patients administered with CTB were mild (arthralgia, headache, and pruritus), and no safety concerns were raised throughout the trial [78].

Interestingly, CTB did not reduce disease severity in an oxazolone-induced colitis model performed by the same group [70]. Oxazolone-induced colitis is mediated by IL-4 driven Th2 cells rather than IL-12/IFN $\gamma$-driven Th1 cells [70]. Thus, it appears that CTB administration had a specific effect on specific $\mathrm{T}$ cell functions involved in TNBS-colitis 
[70]. Although the detailed mechanism by which CTB inhibited Th1 cell was not elucidated, it is possible that the binding of CTB to GM1 ganglioside on immune cells resulted in a signaling cascade of events that led to Th1 inhibition, because non-GM1 binding CTB mutants do not modulate lymphocyte function [79]. In agreement with these findings, CTB decreased monocyte-derived DC maturation and IL-12 production upon LPS stimulation in vitro [80]. Moreover, CTB-pretreated, LPS-stimulated DCs induced low proliferating T cells that had enhanced production of IL-10 and reduced production of IFN $\gamma$. Rouquete-Jazdanian et al. showed that the binding of CTB to GM1 ganglioside directly prevented the activation and proliferation of $\mathrm{CD} 4^{+} \mathrm{T}$ cells [81]. This effect was induced by CTB-mediated sphingomyelinase activation that subsequently increased the production of ceramides, which are known cell cycle arrest inducers [82]. CTB also inhibited protein kinase $\mathrm{C} \alpha$, a pro-growth cellular regulator, which was linked to CTBinduced lipid raft modifications and ceramide-mediated inactivation [83, 84]. 


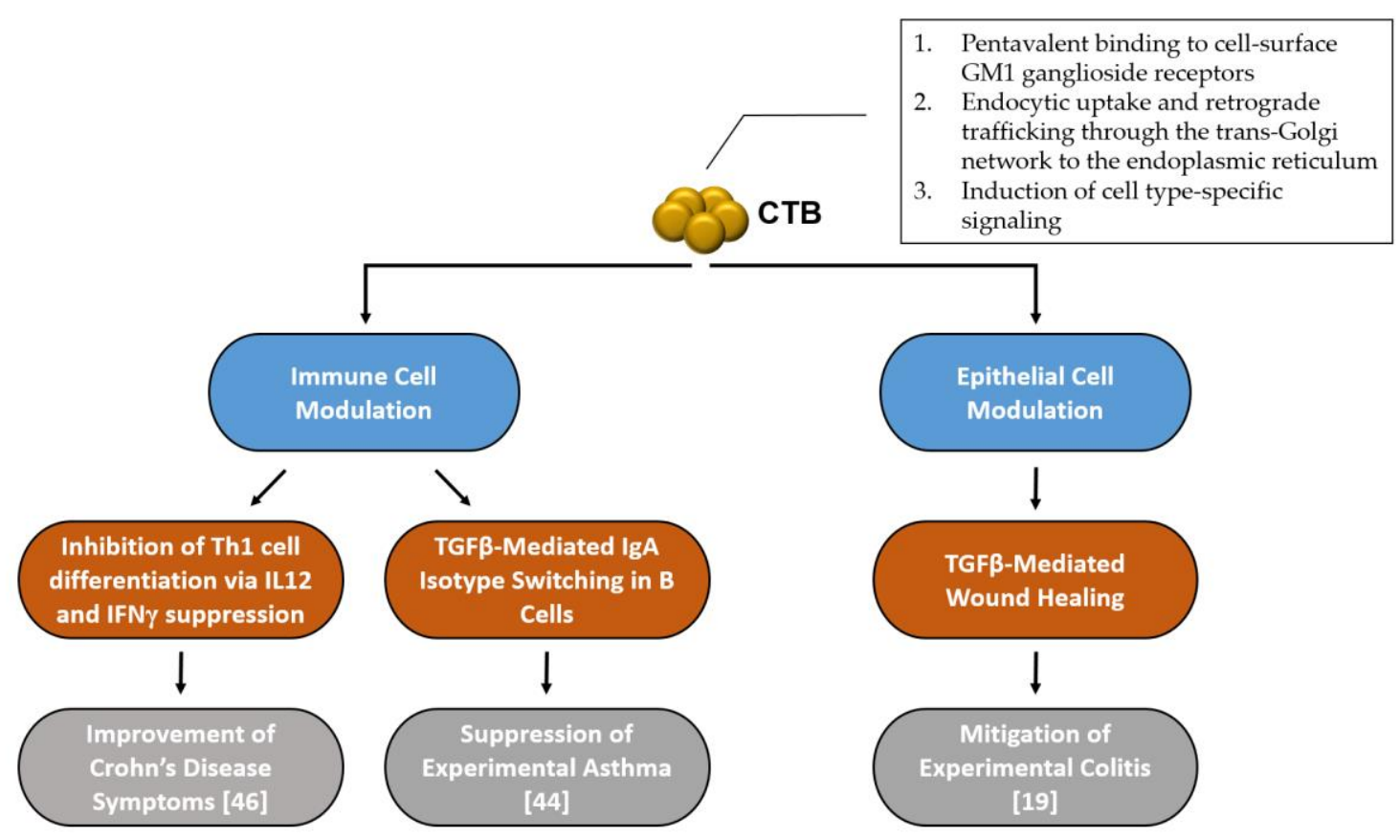

Figure 1. Summary of mechanisms involved in cholera toxin homopentameric B-subunit (CTB)'s inflammatory disease intervention.

\subsubsection{A Plant-Made CTB variant (CTB-KDEL) Directly Modulates Epithelial Cells}

Besides serving as a barrier lining the mucosal surface, epithelial cells have multiple functions associated with the maintenance of gut homeostasis and mucosal healing, and crosstalk between epithelial and immune cells is an important component of those complex biological processes $[85,86]$. Even though CTB first encounters epithelial cells in the gut, the CTB-mediated modulation of epithelial cells and its consequence to the mucosal immune system have largely been ignored in comparison to the protein's direct impacts on immune cells.

In one small study, CTB was shown to induce a dose-dependent increase of IL-10 mRNA levels in the colon epithelial cell-line T84 [87], hinting that CTB could induce epithelial cell-mediated immune modulation [88]. We have recently characterized CTB's 
global impacts on the gut to further our understanding of its unique biological activities. Using CTB-KDEL [42, 89], we have shown that oral administration of the CTB variant significantly altered several immune cell populations in the colon lamina propria [50]. Two-weeks after two oral $30 \mu \mathrm{g}$ CTB-KDEL administrations, Th2 and Treg cells increased in the colon lamina propria. This is not the first report of CTB-induced increase in these cell types [46, 67, 69, 90, 91]. For instance, it has been shown that oral administration of a CTB-insulin conjugate in NOD mice induced a shift from Th1 to Th2 profile while generating Treg cells [46]. Additionally, intraperitoneal administration of nrCTB to rats increased Treg cells in the peripheral blood 24-72 $\mathrm{h}$ after ischemia [90]. Besides the specific $\mathrm{T}$ helper cell subsets, our study has also revealed that innate immune cells_-including dendritic cells, natural killer cells and macrophages (both M1 and M2) - populations were increased in the colon lamina propria two weeks after CTBKDEL oral administration [50]. Furthermore, a global gene expression analysis revealed that CTB-KDEL had more pronounced impacts on the colon than the small intestine, with significant activation of TGF $\beta$-mediated pathways in the colon mucosa [50]. Given that there is a strong link between epithelial-derived TGF $\beta$ and innate immune cells in wound healing [92-94], the results provided implications for the potential utility of CTB-KDEL to promote colonic mucosal health. Subsequently, we found that CTB-KDEL induced TGF $\beta$-mediated wound healing in Caco2 colon epithelial cells. Furthermore, oral administration of CTB-KDEL in mice protected against colon mucosal damage in acute colitis induced by dextran sodium sulfate (DSS). Two oral doses of as low as $1 \mu \mathrm{g}$ of CTB-KDEL mitigated clinical signs of disease (body weight loss, decreased histopathological scores, and blunted escalation of inflammatory cytokine levels) and upregulated wound healing-related genes [50]. Interestingly, CTB-KDEL administration 
prevented fibrosis associated with acute colitis in mice; hence, the protein did not appear to overstimulate TGF $\beta$ signaling, at least under the conditions employed in the study. In fact, TGF $\beta$ gene expression levels were high during the early inflammatory phase and became lower in the recovery phase of the acute colitis model in CTB-KDEL-treated mice.

The main driver of intestinal inflammation in the DSS-colitis model is the damage to the epithelial barrier lining the colon that allows intestinal microbiota into submucosal compartments, in contrast to TNBS-induced colitis [95-99], and meanwhile the therapeutic effects were observed immediately upon CTB-KDEL administration. Therefore, we concluded that CTB-KDEL's protective efficacy in the DSS colitis models were attained by the induction of TGF $\beta$-mediated colonic epithelial wound healing. Given that chronic inflammation and injury of the bowel, such as inflammatory bowel disease (IBD), pose an increased risk of developing colitis-associated colorectal cancer (CAC) $[24,25,100,101]$, CTB-KDEL's effects were also examined in the azoxymethane (AOM)/DSS mouse model of CAC. Biweekly oral administration of CTB-KDEL over 9 weeks significantly reduced inflammation and tumorigenesis in this model, highlighting its therapeutic potential in intestinal injury and inflammatory bowel disease, such as ulcerative colitis (UC) [50]. Therefore, the finding that CTB-KDEL is a topical agent that facilitates TGF $\beta$-based mucosal wound healing in UC and CAC mouse, supports a hypothesis that CTB-KDEL could help address important issues pertinent to therapeutic strategies for mucosal healing in UC.

It is of importance to point out that many of the effects observed in the aforementioned studies using CTB-KDEL may be unique to the plant-made variant, as it has a mutation at amino acid position 4 and an ER retention signal sequence at the $\mathrm{C}$ - 
terminus (N4S-CTB-SEKDEL; [89]). The ER-retention sequence was added to CTBKDEL to improve production in planta, while Asn $4 \rightarrow$ Ser mutation was introduced to avoid $N$-glycosylation $[42,89]$. The addition of the KDEL sequence to N4S-CTB significantly reduced ER stress that otherwise caused poor production yield. It is thought that the KDEL sequence helped prolong CTB-KDEL's residence time in the ER to allow for proper folding and assembly.

The protein ER retention mechanism involving the KDEL receptor is highly conserved among eukaryotic organisms [102]. Thus, there is a possibility that the artificial KDEL sequence of CTB-KDEL may prolong the protein's residence in the epithelial cells upon binding to cell-surface GM1 ganglioside and retrograde transport into the ER, as has been demonstrated for CT $[52,54]$.

Regardless of whether the ER retention signal had a significant contribution to the mucosal healing activity in the mouse colitis models, the study has provided evidence that CTB can exhibit a therapeutic effect against colitis in an epithelia-dependent manner, warranting further investigation of CTB's impacts on epithelial cells.

The overall goal of this project is to develop CTB-KDEL as a biotherapeutic to facilitate mucosal healing in UC patients. To that extent, the objective of this dissertation is to investigate how CTB-KDEL's C-terminal KDEL sequence contributes to the protein's mucosal healing activity in vivo, in vitro, and ex vivo. To achieve this goal I formed three specific aims. First, I aimed to determine if the CTB-KDEL-mediated reduction of colitis we previously reported was unique to the KDEL-tagged protein in a mouse model of DSS-colitis [50], while investigating if CTB-KDEL's epithelial healing activity, intracellular retention, and signaling pathways were affected by the protein's KDEL sequence using a series of CTB-KDEL and CTB analogues. Second, I determined 
if the immunogenicity of CTB-KDEL impacts its immunotherapeutic efficacy and to determine the efficacy of CTB-KDEL in a mouse chronic colitis model. Lastly, I aimed to determine if CTB-KDEL's KDEL sequence contributes to the protein's translational efficacy using a UC patient colon tissue explant.

Collectively, the research in this dissertation characterized CTB-KDEL's colon mucosal healing efficacy, mechanisms, and translatability at cellular and molecular levels, supporting the development of CTB-KDEL as a new topical UC drug. Furthermore, this dissertation discloses previously undescribed impacts of CTB and its derivatives on colon epithelial cells, providing new information to the fields of mucosal immunology and pathophysiology. 


\section{CHAPTER 2: MATERIALS AND METHODS ${ }^{2}$}

CTB variants. Table 1 summarizes CTB variants used in the present study. CTB-KDEL and the non-GM1-binding mutant G33D-CTB-KDEL [50] were produced in $N$. benthamiana and purified to $>95 \%$ homogeneity with an endotoxin level of $<1$ endotoxin units per mg, essentially as described previously $[44,89]$ but with modification in the final chromatography step to selectively purify the proteins with fully intact Cterminus (manuscript in preparation). CTB, CTB-KDE and eCTB-KDEL were produced in E. coli BL21(DE3) according to Hamorsky \& Matoba [103], and purified to > 95\% homogeneity with an endotoxin level of $0.05-0.1$ endotoxin units per mg. Purity and pentamer formation were assessed by SDS-PAGE under denaturing and non-denaturing conditions, whereas the molecular weight of each CTB variant was verified by mass spectrometry, as described before [89].

\footnotetext{
${ }^{2}$ Animals: Eight-week-old C57BL/6J, C3H/HeJ, or Rag $1^{-/-}$female mice were obtained from Jackson laboratories (Bar Harbor, ME). Animal studies were approved by the University of Louisville's Institutional Animal Care and Use Committee.
} 
Caco2 wound healing assay. The $\mathrm{Caco} 2$ wound healing assay was performed as previously described [50]. Briefly, the cells were seeded and grown in complete growth medium (EMEM + 20\% FBS, 1x penicillin-streptomycin) to confluence in 6 well plates (Thermo Scientific Nunc Cell-Culture Treated, Roskilde, Denmark). Afterward, the culture medium was discarded, cells were washed with PBS and then incubated in serumfree media for 6 hours. The cell monolayer was then scratched with a $200 \mu \mathrm{L}$ sterile beveled pipette tip to generate two $0.5-1.0 \mathrm{~mm}$ across linear "wounds" per well and washed with PBS. A test compound (CTB, CTB-KDEL, CTB-KDE or eCTB-KDEL at 0.1-10 $\mu \mathrm{M})$, a positive control ( $0.2 \mathrm{nM}$ [i.e. $5 \mathrm{ng} / \mathrm{mL}$ ] recombinant TGF $\beta 1$; Abcam, Cambridge, MA), a vehicle control (PBS) and/or $4 \mu 8 \mathrm{C}(0.5 \mu \mathrm{M}$; MilliporeSigma, Burlington, MA) were subsequently added in fresh serum-deprived medium. Photomicrographs of the wounds were taken 0,24 , and $48 \mathrm{~h}$ after the wounding at $4 \mathrm{X}$ magnification. Quantification of the remaining cell-free area to the initial wound area was measured using the public domain software Image $\mathrm{J}$ (http://rsbweb.nih.gov), and calculated as a mean percentage ( $\mathrm{n}=2$ experimental replicates) per well. The culture medium/supernatants were collected from each well 24 or $48 \mathrm{~h}$ after wounding and stored at $-80^{\circ} \mathrm{C}$ until analysis. The culture supernatants were analyzed by a human Cytokine/Chemokine or TGFb1, 2, 3 Magnetic Bead Panel (EMD Millipore, St. Charles, MO). The panel was analyzed with a Milliplex MAP Kit on a MagPix with Luminex xMAP technology. Each experiment was performed with 4 biological replicates per construct $(n=4)$. 
Flow cytometry. Flow cytometry was used to assess the binding of CTB and CTBKDEL molecules to Caco2 cells, according to a well-established procedure. Briefly, 1 $\times 10^{5}$ cells were seeded in EMEM (serum free) in the presence of $1 \mu \mathrm{M}$ CTB or CTBKDEL and incubated for 15 minutes on ice. Next, cells were washed and blocked with 3\% BSA (Sigma) on ice. Then cells were incubated with the rat anti-CTB monoclonal antibody 9F9C7 $(1.75 \mu \mathrm{g} / \mathrm{mL})$, which was produced in-house from a rat hybridoma cell line, for $1 \mathrm{~h}$ at room temperature and washed before incubation with a rabbit Alexa FluorTM 488 anti-rat IgG (H+L) (A21210, Life Technologies, Eugen, OR) for $1 \mathrm{~h}$ on ice. Finally, cells were washed and analyzed with a FACSCalibur (Becton Dickinson), counting 10,000 cells per sample. Data were acquired BD FACSCanto II and analyzed with FLOWJO v10 data analysis software, using PBS as a negative control $(n=4)$.

Caco2 cell immunofluorescence. Cells were seeded 2 x $10^{5}$ cells/well in Lab-Tek II chamber slides (Thermo, Rochester, NY) and grown for $72 \mathrm{~h}$ in complete growth medium. The media was subsequently removed, and cells were washed with PBS followed by a test compound (CTB or CTB-KDEL at $1 \mu \mathrm{M})$ or a vehicle control treatment in fresh serum-deprived medium. Cells were incubated for $24 \mathrm{~h}$ at $37^{\circ} \mathrm{C}$ in a humidified $5 \% \mathrm{CO}_{2}$ then fix, permeabilized, and stained. Briefly, after treatment cells were washed with PBS, they were fixed in $0.4 \mathrm{~mL} 4 \%$ paraformaldehyde in PBS for $15 \mathrm{~min}$ at room temperature. Then cells were permeabilized with $0.4 \mathrm{~mL} 0.2 \%$ TitonX-100 in PBS for an additional 15 min at room temperature. Subsequently, cells were blocked (3\% BSA, Sigma) and treated with $4.4 \mu \mathrm{g} / \mathrm{mL}$ of the anti-CTB monoclonal antibody 9F9C7 and 1 $\mu \mathrm{g} / \mathrm{mL}$ of rabbit anti-KDEL receptor pan $\mathrm{pAb}$ (Thermo PA5-67422) for $3 \mathrm{~h}$ at room 
temperature. After subsequent washing (PBS), an anti-Rat IgG $(\mathrm{H}+\mathrm{L})$ Cross-Adsorbed Secondary Antibody, Alexa Fluor 488 (Thermo) and anti-Rabbit preadsorbed IgG H\&L (Alexa Fluor® 555) was applied to cells for $3 \mathrm{~h}$ at room temperature followed by additional washing with PBS. Cells were then mounted with coverslips using mounting medium for fluorescence with DAPI (VECTASHIELD®, Burlingame, CA). The proximity ligation assay (Duolink® PLA, Sigma) was performed according to the manufacturer's protocol using the same fixation/permeabilization and primary antibody application protocol as described above using $1 \mu \mathrm{g} / \mathrm{mL}$ of rabbit anti-KDEL receptor pan pAb (Thermo PA5-67422) and 1:200 goat anti-CTB pAb (Emdmillipore). Slides were analyzed by wide-field fluorescence confocal microscope (60x magnification, Z-stacked images). Co-localization statistical analysis was performed using Imaris software (Bitplane), values were calculated in PLA signals per cell.

Co-immunoprecipitation. Caco2 cells $\left(1 \times 10^{6}\right)$ were seeded in $10 \mathrm{~cm}^{2}$ plate and incubated in EMEM with $20 \%$ of FBS for 48 hours. After changing the medium to EMEM without FBS supplement, $1 \mu \mathrm{M}$ of G33D-CTB-KDEL, CTB or CTB-KDEL, or a vehicle control (PBS) were added to the cells and incubated for $24 \mathrm{~h}$. Cells were washed with PBS and cell lysates were prepared in $500 \mu \mathrm{L}$ of T-PER buffer (Thermo fisher scientific) supplied with 1x protease, phosphatase inhibitor (Thermo fisher scientific). After centrifugation at $13,000 \mathrm{~g}$ for 10 minutes, supernatants were mixed with $4 \mu \mathrm{g}$ of an anti-CTB antibody (7A12B3, which was produced in-house from a rat hybridoma cell line) or anti-BiP antibody (Cell Signaling Technology, Danvers, MA) or an anti-IRE1 $\alpha$ antibody (Cell Signaling Technology). After incubation at $4{ }^{\circ} \mathrm{C}$ for $24 \mathrm{~h}, 10 \mu \mathrm{L}$ of Protein 
A beads (Santa Cruz Biotechnology; Dallas, TX) were added. After additional incubation at $4{ }^{\circ} \mathrm{C}$ for $2 \mathrm{~h}$, the mixture was washed with T-PER buffer. The immunoprecipitated proteins were detected by an immunoblot analysis using $1 \mu \mathrm{g} / \mathrm{mL}$ of the rat anti-CTB antibody 7A12B3 (produced in-house), $2 \mu \mathrm{g} / \mathrm{mL}$ of an anti-KDEL receptor (KDELR) antiserum (Abcam), $2 \mu \mathrm{g} / \mathrm{ml}$ of an anti-BiP antibody (Cell Signaling Technology) or 2 $\mu \mathrm{g} / \mathrm{mL}$ of an anti-IRE1 $\alpha$ antibody (Cell Signaling Technology).

XBP knockdown with siRNA. In a 6-well tissue culture plate, 2 x $10^{5}$ Caco2 cells were seeded per well in $2 \mathrm{ml}$ of EMEM with 20\% FBS and 1x penicillin-streptomycin. Small interfering RNA (siRNA) was used to silence XBP1. The human XBP1 siRNA and scrambled sequences siRNA (control) were purchased from Santa Cruz Biotechnology. When cells were $60-80 \%$ confluent, $1 \mu \mathrm{g}$ of siRNAs was transfected by lipofectamine transfection reagents (Santa Cruz Biotechnology). Subsequently, the wound healing assay was performed as described above, and total RNA was isolated by RNeasy Microarray Tissue Kit from Qiagen to verify XBP1 levels using the XBP-1 (h) primer set supplied from Santa Cruz Biotechnology.

Immunoblot analysis. When Caco2 cells were 60-80\% confluent in EMEM supplemented with 20\% FBS and 1x penicillin-streptomycin, the medium was changed to EMEM without FBS supplement and treated $1 \mu \mathrm{M}$ of G33D-CTB-KDEL, CTB or CTBKDEL, or a PBS vehicle control. After $24 \mathrm{~h}$ incubation, cell lysates were prepared with T-PER buffer supplied with 1x protease, phosphatase inhibitor (Thermo Fisher Scientific). Cell debris were removed by centrifugation at $13,000 \mathrm{~g}$ for $10 \mathrm{~min}$ at $4^{\circ} \mathrm{C}$. 
One hundred $\mu \mathrm{g}$ of total proteins were loaded for SDS-PAGE analysis, using a $10 \%$ of Bolt Bis-Tris Plus gel (Thermo Fisher Scientific) to separate large-size phosphorylated proteins in MES-based running buffer. To analyze the intracellular stability of CTB and CTB-KDEL, Caco2 cells were incubated $4 \mathrm{~h}$ with $2 \mu \mathrm{M}$ of CTB or CTB-KDEL, washed and immediately analyzed or incubated in EMEM for additional 12 or $24 \mathrm{~h}$ before analysis by immunoblot using $1.75 \mu \mathrm{g} / \mathrm{mL}$ of the rat anti-CTB monoclonal antibody 7A12B3. To detect UPR markers, we used $2 \mu \mathrm{g} / \mathrm{mL}$ of an anti-BiP antibody (Cell Signaling Technology), an anti-IRE1 $\alpha$ antibody (Cell Signaling Technology) or an antiPERK antibody (Cell Signaling Technology). Loading control was $\beta$-actin, which was detected by $1 \mu \mathrm{g} / \mathrm{mL}$ of an anti- $\beta$-actin antibody (Santa Cruz Biotechnology). For mouse primary colon epithelial cells, cells were isolated from $3-6 \mathrm{~cm}$ of the colon from four mice. Colons were cut open lengthwise, washed, and then cut into $2 \mathrm{~mm}$ pieces. The tissues were washed 20 times in cold PBS followed by a 20 min incubation at room temperature in $50 \mathrm{~mL}$ conical tubes containing $30 \mathrm{ml}$ of pre-warmed CMF HBSS $/ 0.1 \%$ FBS and 2mM EDTA on a rocking platform at $20 \mathrm{rmp}$. The tissues were allowed to settle by gravity for 30 seconds and then the supernatant was discarded. To separate epithelial cells, the tissues were resuspended in $10 \mathrm{~mL}$ cold $\left(4^{\circ} \mathrm{C}\right) \mathrm{CMF} \mathrm{HBSS} / 1.5 \% \mathrm{FBS}$ and pipetted up and down three times viciously. The intestinal pieces were allowed to settle and then the supernatant was filtered through a $70 \mu \mathrm{m}$ filter. This cell separation step followed by filtration was repeated 3 times creating four fractions of filtered supernatants. Fractions 3 and 4 were combined and centrifuged at $300 \mathrm{x} g$ for five minutes at $4^{\circ} \mathrm{C}$. Supernatants were carefully discarded and the pellet was resuspended in $10 \mathrm{~mL}$ cold $\left(4^{\circ} \mathrm{C}\right) \mathrm{EMEM}$ with $20 \% \mathrm{FBS}$ followed by a subsequent $200 \mathrm{x} \mathrm{g}$ centrifugation. Finally the cells were resuspended in EMEM with 20\% FBS, counted, and 
plated. Thereafter, the mouse primary colon epithelial cells were subjected to the same immunoblot analysis protocol as mentioned above for Caco2 cells.

The DSS-induced acute colitis study. For the acute DSS recovery model of intestinal injury, 10 mice per group, randomly assigned, were used. DSS exposure was initiated on the day mice turned 9 weeks old (day 0), using a modified method [50]. Body weights were measured at the initiation of DSS exposure as a baseline and every morning thereafter to determine percent change. Animals received 3\% DSS (M.W. 36,000 to 50,000; MP Biomedicals, Santa Ana, CA) in drinking water for 7 days. On the 7 th day of DSS exposure, animals gavaged with PBS, CTB or CTB-KDEL after sodium bicarbonate administration, as described previously [89], and allowed to recover 7 days during which the animals received normal drinking water.

Histology. Colons were removed and washed with PBS. A portion of the distal colon was fixed with paraformaldehyde overnight and stored in $70 \%$ ethanol until paraffin embedding, sectioning and routine H\&E staining. Inflammation scoring was performed as previously described [50]. Tissue sections from 10 mice were scored in a blinded manner and averaged for each group.

Immunohistochemistry (IHC). Colons were removed and washed with PBS ( $\mathrm{n}=10)$. A portion of the distal colon was fixed with paraformaldehyde overnight and stored in $70 \%$ ethanol until paraffin embedding and sectioning. Sections were deparaffinized with 
Citrisolv and rehydrated through several ethanol washing steps ending with incubation in distilled water. Antigen retrieval was performed overnight with a 2100 Retriever (Electron Microscopy Sciences) using a pH 6.0 buffer. Tissue sections were blocked for endogenous peroxidase, avidin, biotin, and serum from the animal in which the secondary antibody was raised. Primary antibody (anti-E-cadherin 1:100; Abcam) was incubated with the tissue sections for $1 \mathrm{~h}$ at room temperature. The Vectastain Elite ABC kit (goat anti-rabbit; Vector Labs, Burlingame, CA) was used to label the primary antibody. Ecadherin+ cells were visualized with the ImmPACT DAB Substrate Kit (Vector Labs) and then dehydrated through an ethanol gradient and finally incubated with Citrisolv. Sections were scanned using an Aperio ScanScope CS (Leica Biosystems, Buffalo Grove, IL).

RNA isolation. Distal colon tissue ( 14 mg) from each animal, initially stored in RNAlater (Qiagen, Valencia, CA) at $-20{ }^{\circ} \mathrm{C}$, was placed in QIAzol lysis reagent and homogenized. An RNeasy Microarray Tissue Kit from Qiagen was used to purify the RNA from the tissue homogenate. RNA concentration, quality and purity were confirmed by spectrophotometer then stored at $-80^{\circ} \mathrm{C}$ until use.

qRT-PCR. First strand cDNA was obtained from reverse transcription of 100 ng RNA using a SUPERSCRIPT VILO cDNA synthesis kit (Life Technologies, Carlsbad, CA) according to the manufacturer's instructions. Template cDNA were added to a reaction mixture containing $\mathrm{RT}^{2}$ SYBR Green Master Mix (Qiagen) and loaded in $\mathrm{RT}^{2}$ Profiler PCR Array Standard 96 well Plates (Qiagen). These plates contain pre-spotted individual 
gene expression probes for the detection of genes of interest as well as the house keeping genes $18 S, A C T B$ and GAPDH. Gene quantification was carried out on a 7500 Fast RealTime PCR System (Applied Biosystems) with the following conditions: 1 cycle (10 min at $\left.95^{\circ} \mathrm{C}\right) ; 40$ cycles $\left(15 \mathrm{~s}\right.$ at $95^{\circ} \mathrm{C} ; 1 \mathrm{~min}$ at $60^{\circ} \mathrm{C}$ ). The 7500 Software v2.0.6 (Applied Biosystems) was used to determine the cycle threshold (Ct) for each reaction and derive the expression ratios relative to control. Wound healing pathway analysis was performed with a RT2 Profiler PCR Mouse Wound Healing Array (Qiagen, Manchester, UK) under the same conditions described above.

\section{Treatment and culturing of colon explants obtained from ulcerative colitis patients.}

All research involving human tissue was performed in accordance with relevant guidelines and regulations established by the University of Louisville Institutional Review Board Committee. The treatment and culturing of colon explants was performed using an immersion culturing system developed from a previously-described methods [77, 104, 105]. Colorectal tissues were obtained from consenting patients at the time of colectomy. Immediately after excision, colectomy tissue was placed in complete medium (RPMI 1640 supplemented with $2 \mathrm{mM}$ L-glutamine, $10 \mathrm{mM}$ HEPES buffer, $10 \mu \mathrm{g} / \mathrm{mL}$ gentamicin, $100 \mathrm{U} / \mathrm{mL}$ each of penicillin and streptomycin, and 10\% FBS (Hy-Clone)) on ice and transported to the lab. The tissue was immediately divided and placed in organ culture dishes at $37^{\circ} \mathrm{C}$ in a humidified $5 \% \mathrm{CO}_{2}$. The tissues were placed luminal side up in $2 \mathrm{~mL}$ complete growth medium. Cultures were incubated with or without the addition of PBS, CTB, or CTB-KDEL $(1 \mu \mathrm{M})$ at $37^{\circ} \mathrm{C}$ for $24 \mathrm{~h}$. Thereafter, the supernatant was aliquoted and stored at $-80^{\circ} \mathrm{C}$, tissues were washed in complete medium and 
homogenized for gene expression analysis, formalin-fixed for histopathology, or frozen in liquid nitrogen for additional analysis as necessary.

Statistics. For all data, outliers were determined by statistical analysis using the Grubb's test $(P<0.05)$ and excluded from further analysis. Graphs were prepared and analyzed using GraphPad Prism version 5.0 (GraphPad Software, La Jolla, CA). To compare two data sets, an unpaired, two-tailed Student's $t$ test was used. To compare three or more data sets, one-way ANOVA with Bonferroni's multiple-comparison post-test or KruskalWallis test with Dunn's multiple comparison post-test were performed. For body weights and DAI results, a two-way ANOVA with Bonferroni's multiple-comparison post-test was employed. A $P$ value of $<0.05$ was considered significant. 
CHAPTER 3: A MODIFIED CHOLERA TOXIN B SUBUNIT CONTAINING AN ER RETENTION MOTIF ENHANCES COLON EPITHELIAL REPAIR VIA AN UNFOLDED PROTEIN RESPONSE ${ }^{3}$

\subsection{Introduction}

Cholera toxin (CT) is classified as an AB5 toxin composed of one A subunit (CTA) and a pentameric B subunit (CTB) $[106,107]$. In the gut, CT binds to GM1 ganglioside, via CTB, located on the plasma membranes of epithelial cells that line the luminal surface [36-39]. CTB contains five GM1 binding sites that lie on the outer edge of each B subunit $[28,53]$. Once CT is bound to GM1 ganglioside (up to five gangliosides at once), it is endocytosed and trafficked via retrograde transport to the endoplasmic reticulum (ER) [52]. Once in the ER, the catalytic domain of CTA (CTA1) is dissociated from CTB by protein disulfide isomerase. Subsequently, CTA1 enters the cytosol via the ER-associated degradation pathway and returns to the inner surface of the plasma membrane to cause water secretion and diarrhea [28, 51, 107]. Meanwhile the fate of CTB after dissociation with CTA1 in the ER is not well understood. We have recently created a variant of CTB with a C-terminal ER retention motif (CTB-KDEL; previously termed CTBp; [89]), which was demonstrated to possess GM1-binding affinity and oral

\footnotetext{
${ }^{3}$ Modified from: FASEB J. 2019 Sep 27:fj201901255R. doi: 10.1096/fj.201901255R.
} 
immunogenicity that are virtually equivalent to CTB. Additionally, we found that CTBKDEL could mitigate dextran sodium sulfate (DSS)-induced acute colitis and reduced tumor development in an azoxymethane (AOM)/DSS model of colitis associated colon cancer in mice [50]. These effects were associated with increased TGF $\beta$ expression and activation, although the mechanism by which CTB-KDEL upregulated TGF $\beta$ remained elusive. While investigating the mechanistic principle, we recently discovered that the epithelial wound repair effects observed in the aforementioned studies are unique to CTB-KDEL and not attained by CTB. The ER-retention motif was initially added to CTB to improve recombinant production in tobacco plants (Nicotiana benthamiana), which aided in reducing ER stress that otherwise caused poor production yield [42, 89]. It was thought that the KDEL sequence helped prolong CTB-KDEL's residence time in the ER within plant cells to allow for proper folding and assembly. Additionally, the protein ER retention mechanism involving the KDEL receptor is highly conserved among eukaryotic organisms [102]. Thus, the plant-made protein's C-terminal ER retention signal sequence KDEL, added to improve the protein's production in planta, could theoretically alter the protein's fate upon entering mammalian cells $[52,54,107]$, which in turn might explain the unique colon mucosal healing activity of CTB-KDEL.

Here we investigated how the artificial ER-retention motif of CTB-KDEL contributes to the protein's mucosal healing activity in in vitro cell culture and in vivo mouse DSS acute colitis. The results have led to the discovery that an unfolded protein response (UPR) plays a critical role in the induction of intestinal mucosal healing, providing implications for novel therapeutic approaches for IBD. 


\section{Table 1.}

$\underline{\text { Recombinant CTB variants used in the present study }}$

\begin{tabular}{|l|l|l|}
\hline Protein Name & C-terminal KDEL sequence & Production System \\
\hline CTB & No & Escherichia coli BL21(DE3) \\
\hline CTB-KDEL & Yes & Nicotiana benthamiana \\
\hline CTB-KDE & $\begin{array}{l}\text { Lacking the terminal Leu } \\
\text { residue }\end{array}$ & Escherichia coli BL21(DE3) \\
\hline eCTB-KDEL & Yes & Escherichia coli BL21(DE3) \\
\hline G33D-CTB-KDEL & Yes & Nicotiana benthamiana \\
\hline
\end{tabular}

\subsection{Results}

\subsubsection{The C-Terminal KDEL Sequence is Essential for the Colon Epithelial Repair} Activity of CTB-KDEL.

To first determine if the previously described colon mucosal healing effect is unique to CTB-KDEL, we employed a human colon epithelial cell line Caco2 model of wound healing [50]. Cell migration (i.e., "wound closure") over $48 \mathrm{~h}$ was tested using 3 $\mu \mathrm{M}$ of CTB-KDEL, CTB, or PBS. As shown in Figure 2A-C, the results revealed that CTB had no effect beyond the natural healing response noted in the PBS-treated group, while CTB-KDEL treatment significantly enhanced wound closure both at 24 and $48 \mathrm{~h}$ post wounding similarly to the recombinant TGF $\beta$ positive control (Figure 2B). This enhanced cell migration effect was observed in a broad concentration range of CTBKDEL, from $3 \mu \mathrm{M}$ down to $0.1 \mu \mathrm{M}$. On the other hand, CTB had no effect at the same 
concentrations tested (Figure 2C). We have previously demonstrated in the same model that CTB-KDEL's cell migration effect is mediated by TGF $\beta$, as an anti-TGF $\beta$ antibody completely inhibited the effect [50]. Thus, TGF $\beta$ levels in the culture supernatants were measured using a multiplex bead array. In contrast to CTB, CTB-KDEL significantly increased TGF $\beta 1$ and TGF $\beta 2$ levels 24 hours post-wounding (Figure 2D). To rule out the possibility that the difference observed between CTB-KDEL and CTB was the result of differential binding to $\mathrm{Caco} 2$ cells, we performed a flow cytometry analysis where Caco2 cells were treated with $1 \mu \mathrm{M}$ CTB-KDEL or CTB. Both CTB-KDEL and CTB showed high and similar binding, with $>75 \%$ of Caco2 cells showing positive (Figure 2E). Consistent with these results, CTB-KDEL and CTB have shown comparable GM1binding affinity in ELISA, surface plasmon resonance, and flow cytometry analysis using the mouse macrophage cell line RAW264.7 [89]. Together, these results indicate that CTB-KDEL, but not CTB, facilitates TGF $\beta$-mediated epithelial wound healing in the Caco2 model. Since CTB-KDEL and CTB equally bound to the epithelial cell, the discrepancy can be attributed to distinct intracellular events, likely triggered upon their internalization and retrograde transportation. 

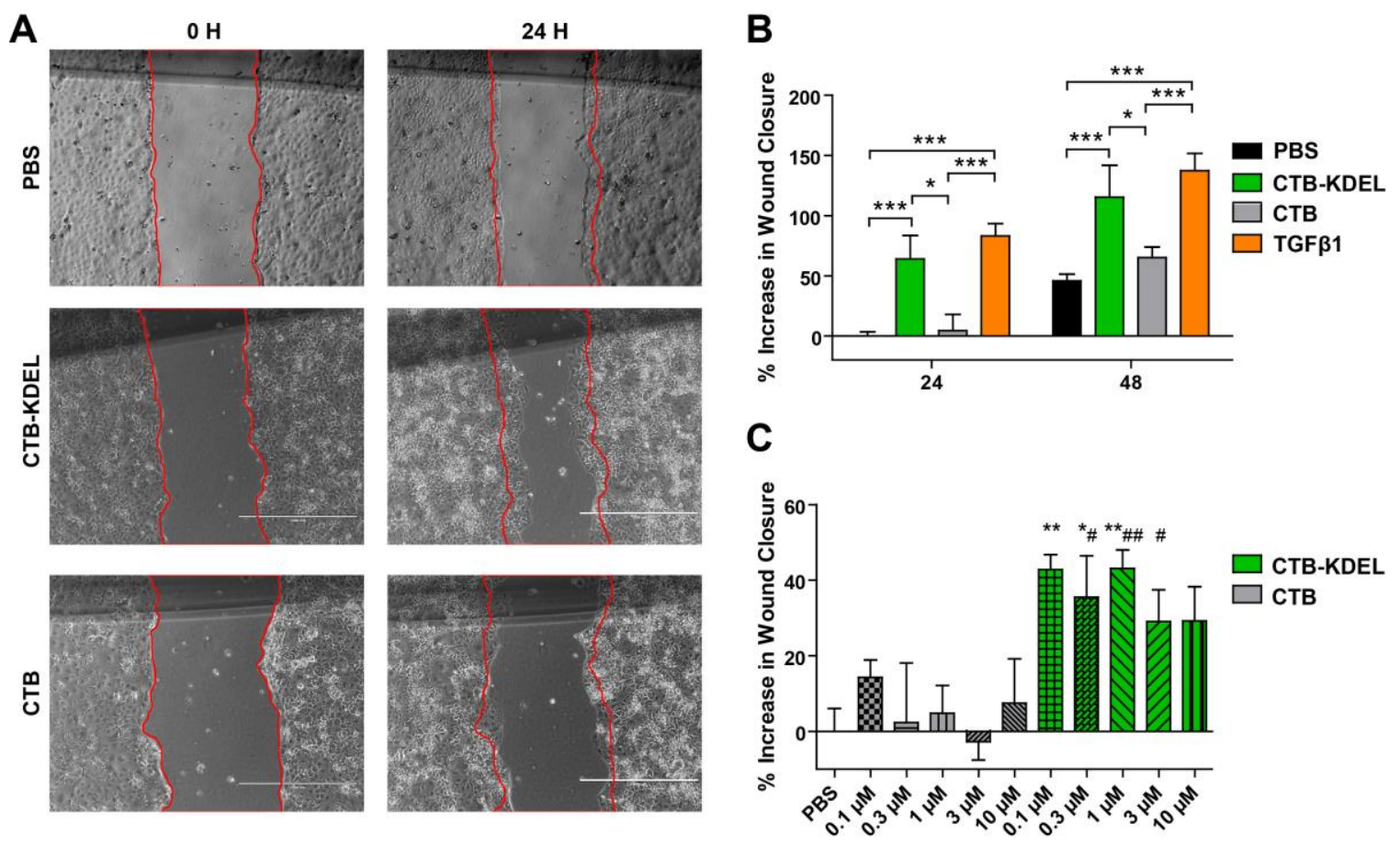

D
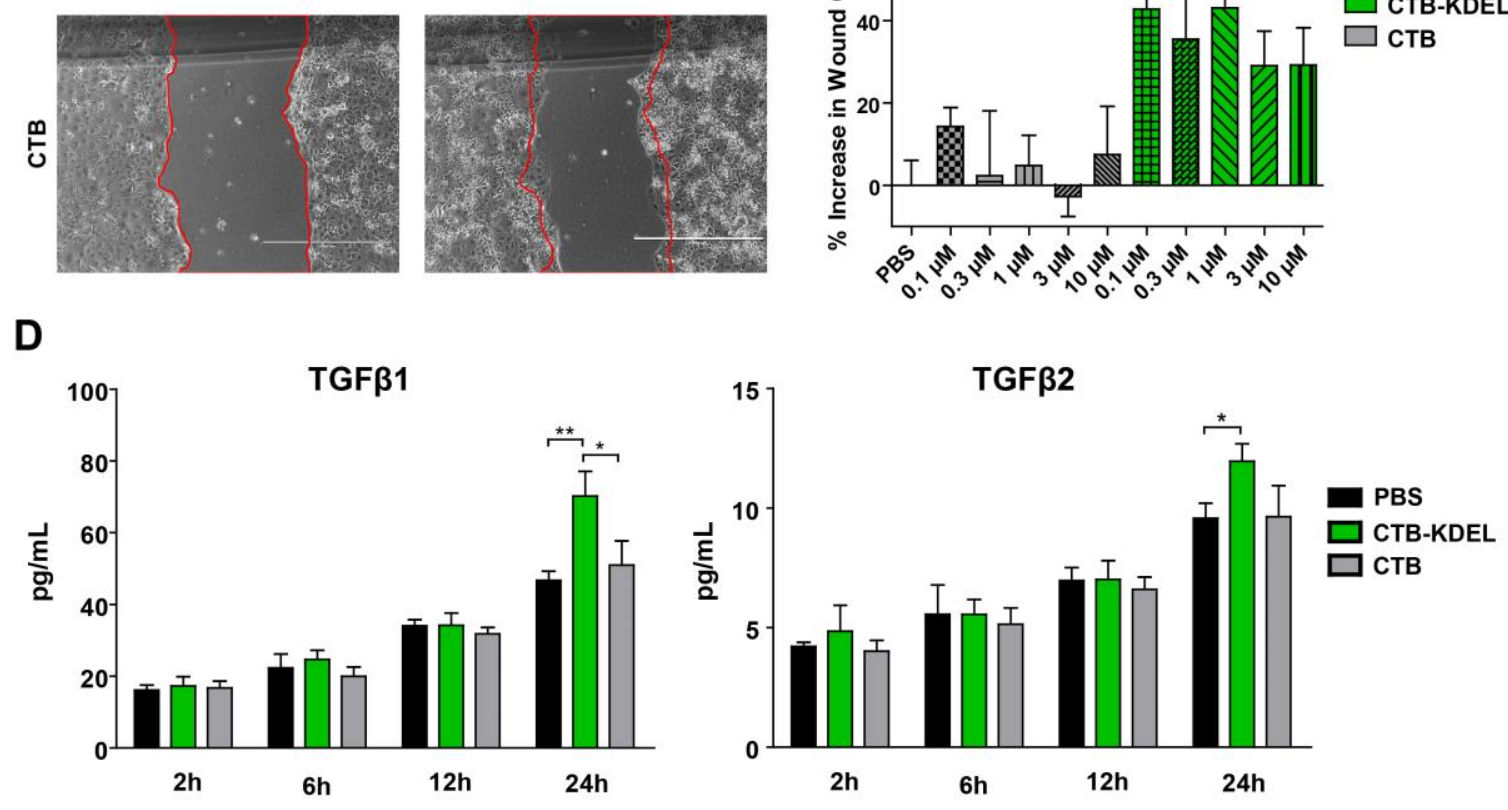

$\mathbf{E}$

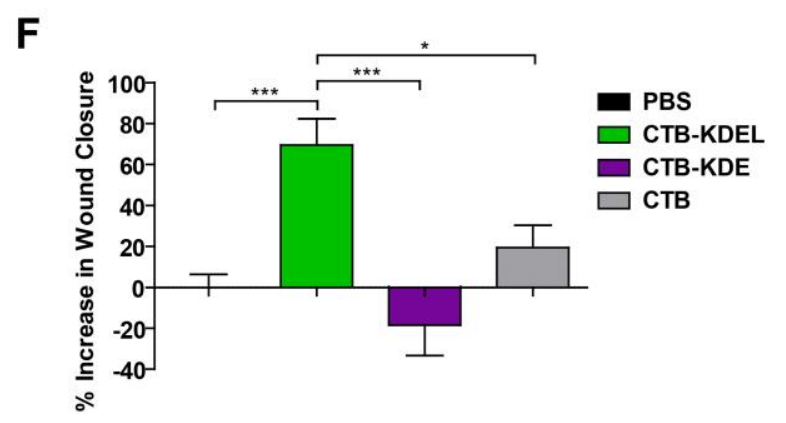

Figure 2. CTB-KDEL, but not CTB, enhances cell migration and TGFß levels in a human colon epithelial cell wound healing model. Caco2 cells were grown to confluence and scratched with a pipette tip. Cells were then incubated with PBS, CTB, CTB-KDEL, or TGF $\beta 1$. The in vitro wound closure was recorded over $48 \mathrm{~h}$ and $4 \mathrm{x}$ 
magnification images were acquired with the EVOS® FL imaging system (Advanced Microscopy Group) and mean percentage closure was determined by Image $\mathbf{J}$ software. (A) Representative photomicrographs of Caco2 cells treated with PBS, CTB-KDEL or CTB at 0 and $24 \mathrm{~h}$ post scratch. (B) Analysis of "wound closure" induced by $3 \mu \mathrm{M} \mathrm{CTB}$ KDEL or CTB, or $0.2 \mathrm{nM}$ (or $5 \mathrm{ng} / \mathrm{mL}$ ) TGF $\beta 1$ over $48 \mathrm{~h}$, compared to a PBS vehicle control. (C) Percent increase in "wound closure" by varying concentrations of CTB or CTB-KDEL compared to PBS after $24 \mathrm{~h}$. (D) TGF $\beta 1$ and TGF $\beta 2$ protein concentrations in Caco2 cell supernatants. (E) Caco2 cell flow cytometry analysis. Cells $\left(1 \times 10^{5}\right)$ were treated with $1 \mu \mathrm{M}$ CTB or CTB-KDEL, or PBS for 15 min on ice, then fix and stained with a rat anti-CTB monoclonal antibody. (F) Percent increase in "wound closure" by 1 $\mu \mathrm{M}$ of CTB-KDEL, CTB-KDE or CTB compared to PBS after $24 \mathrm{~h}$. Bars represent mean \pm SEM of four independent analyses. One-way ANOVA with Bonferroni's multiple comparison tests was used to compare all pairs of groups (B - E). Significantly different pairs are highlighted with asterisks $(*, * *, * * * \mathrm{P}<0.05,0.01,0.001)$.

The above results led us to hypothesize that the C-terminal KDEL sequence is essential to CTB-KDEL's wound healing activity. To further delineate the importance of the ER retention motif, a variant lacking the C-terminal Leu residue (CTB-KDE) was made. As shown in Figure 2F, CTB-KDE failed to promote wound healing unlike CTBKDEL in a Caco2 cell wound healing assay. Additionally, in order to rule out the possibility that the unique activity is not attributed to the Nicotiana benthamiana production system, CTB-KDEL was produced in Escherichia coli (eCTB-KDEL) and tested in the Caco2 cell wound healing assay. Indeed, e CTB-KDEL significantly 
promoted wound healing 24 hours post-wounding in contrast to the non-KDEL counterpart (Figure 3).

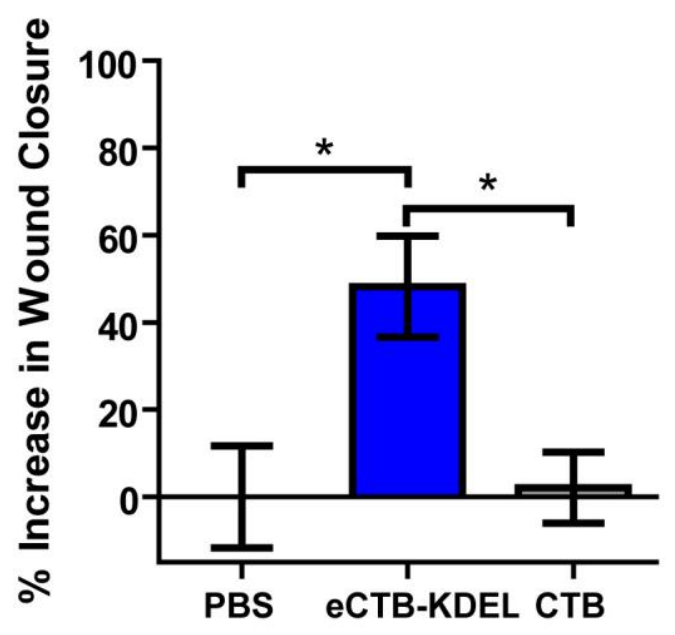

Figure 3. Escherichia coli produced CTB-KDEL enhances cell migration. Caco2 cells were grown to confluence and scratched with a pipette tip. Cells were then incubated with PBS, eCTB-KDEL, or CTB. The in vitro wound closure was recorded over $24 \mathrm{~h}$ and 4x magnification images were acquired with the EVOS® FL imaging system (Advanced Microscopy Group) and mean percentage closure was determined by Image $\mathbf{J}$ software 
A

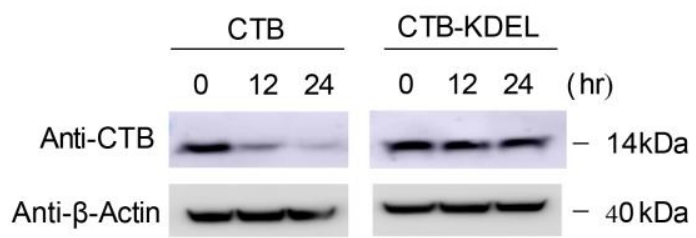

B

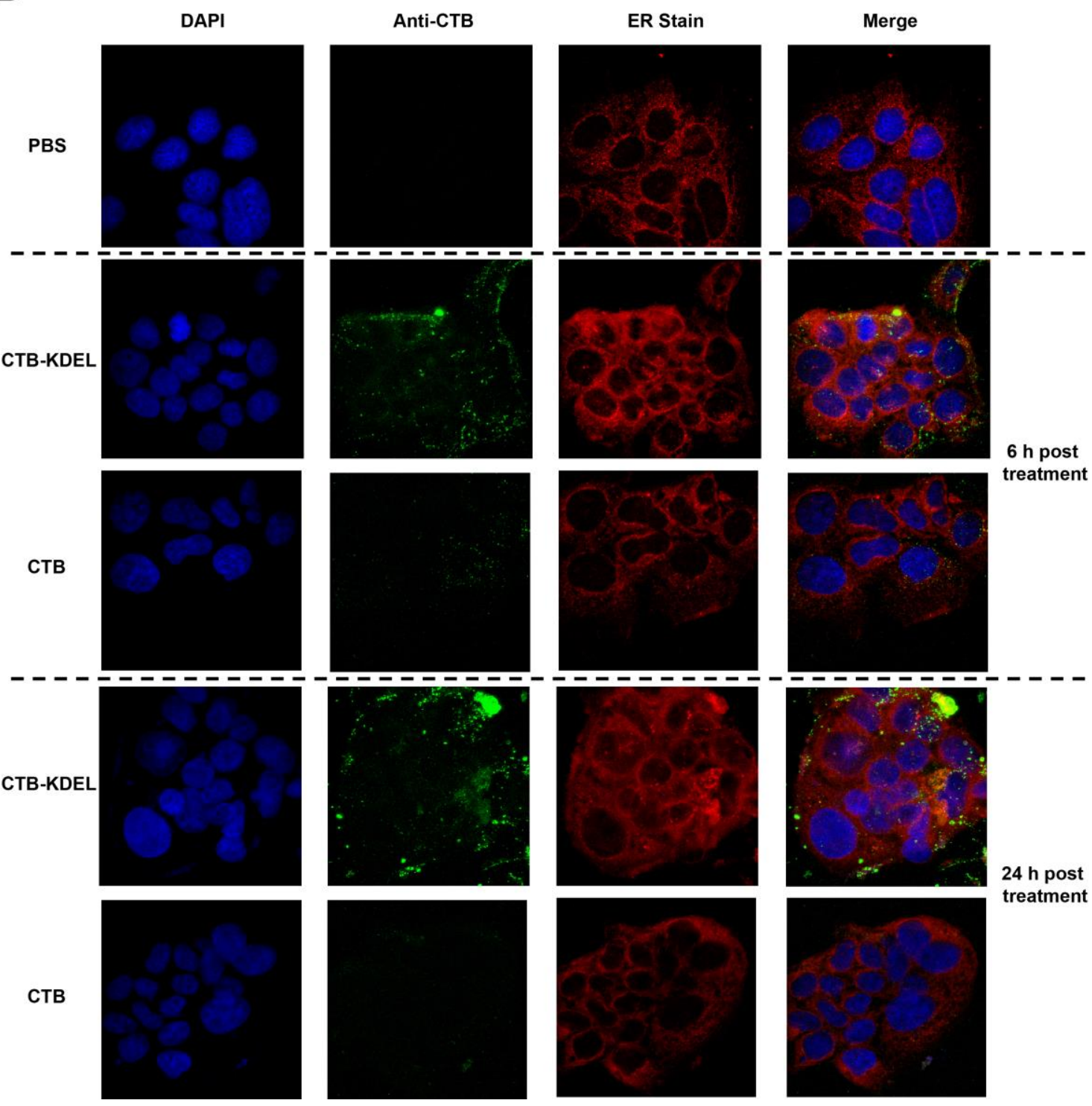

Figure 4. Stability of CTB and CTB-KDEL in Caco2 cells. (A) After $4 \mathrm{~h}$ incubation with $2 \mu \mathrm{M}$ of CTB or CTB-KDEL, Caco2 cells were washed with EMEM, and then, cells were lysed at different timings ( 0,12 and $24 \mathrm{~h})$. Anti-CTB antibodies showed CTB and CTB-KDEL proteins in western blot (Anti-CTB) and actin antibodies detected actin 
proteins (Anti- $\beta$-Actin). (B) Immunofluorescence analysis of CTB-KDEL or CTB intracellular localization within the ER. CTB-KDEL accumulates within Caco2 cells in contrast to CTB. Caco2 Cells were treated with $1 \mu \mathrm{M}$ PBS, CTB, or CTB-KDEL for 6 or 24 hours. Cells were fixed/permeabilized and stained with anti-CTB mAb detected by Alexa FluorTM 488 (green), ER-selective red dye (red), and DAPI (blue). Slides were analyzed by wide-field fluorescence confocal microscope (60x magnification, Z-stacked images). Scale bars $=20 \mu \mathrm{m}$. (A) Performed by Dr. Youngjun Oh

We next explored if the ER retention motif of CTB-KDEL has any influence on the protein's residence within epithelial cells. Due to the known function of KDEL receptors (KDELR) [108-110], it was hypothesized that CTB-KDEL could localize and remain in the ER for an extended period after retrograde transport [54]. To test this hypothesis, Caco2 cells were treated with CTB-KDEL or CTB then washed and incubated for $0,12,24$ hours, followed by an immunoblot analysis to detect CTB and CTB-KDEL in the cell lysates. As shown in Figure 4A, CTB-KDEL was continuously detected in the cell lysates over $24 \mathrm{~h}$, whereas CTB showed decreasing levels, with only a marginal amount detected at $24 \mathrm{~h}$ post treatment. To discern the location of CTB-KDEL and CTB in the cells, we utilized confocal microscopy, in which cells were treated with CTB-KDEL or CTB for $24 \mathrm{~h}$ and then fixed and stained for CTB, the ER, the KDELR and nuclei. As depicted in Figure 4B, CTB-KDEL was detected much more prevalently inside Caco2 cells at 6 and 24 h post treatment. Since CTB-KDEL and CTB showed similar binding to the surface of Caco2 cells (Figure 2E), it is likely that the difference in their amounts detected within Caco2 cells occurred after internalization. Confocal microscopy showed the colocalization of CTB-KDEL and KDELR $24 \mathrm{~h}$ post treatment, 
while CTB was hardly detected (Figure 5A). This was also corroborated by a proximity ligation assay (PLA), which reveals protein-protein interactions that are within a $40 \mathrm{~nm}$ proximity [111] (Figure 5B \& C). The interaction of CTB-KDEL, CTB-KDE or CTB with KDELR in Caco2 cells was also confirmed by co-immunoprecipitation (Figure 5D). Rat anti-CTB antibody slightly preferred to original CTB than CTB-KDE and CTBKDEL. It seems to cause by structural and electronic hindrances from positive and negative charged residues in KDE. While CTB-KDEL showed clear binding to the KDELR, such interaction was not observed with CTB or CTB-KDE. Collectively, these results demonstrate that CTB-KDEL has a prolonged residence within Caco2 cells due to its interaction with the KDELR. 


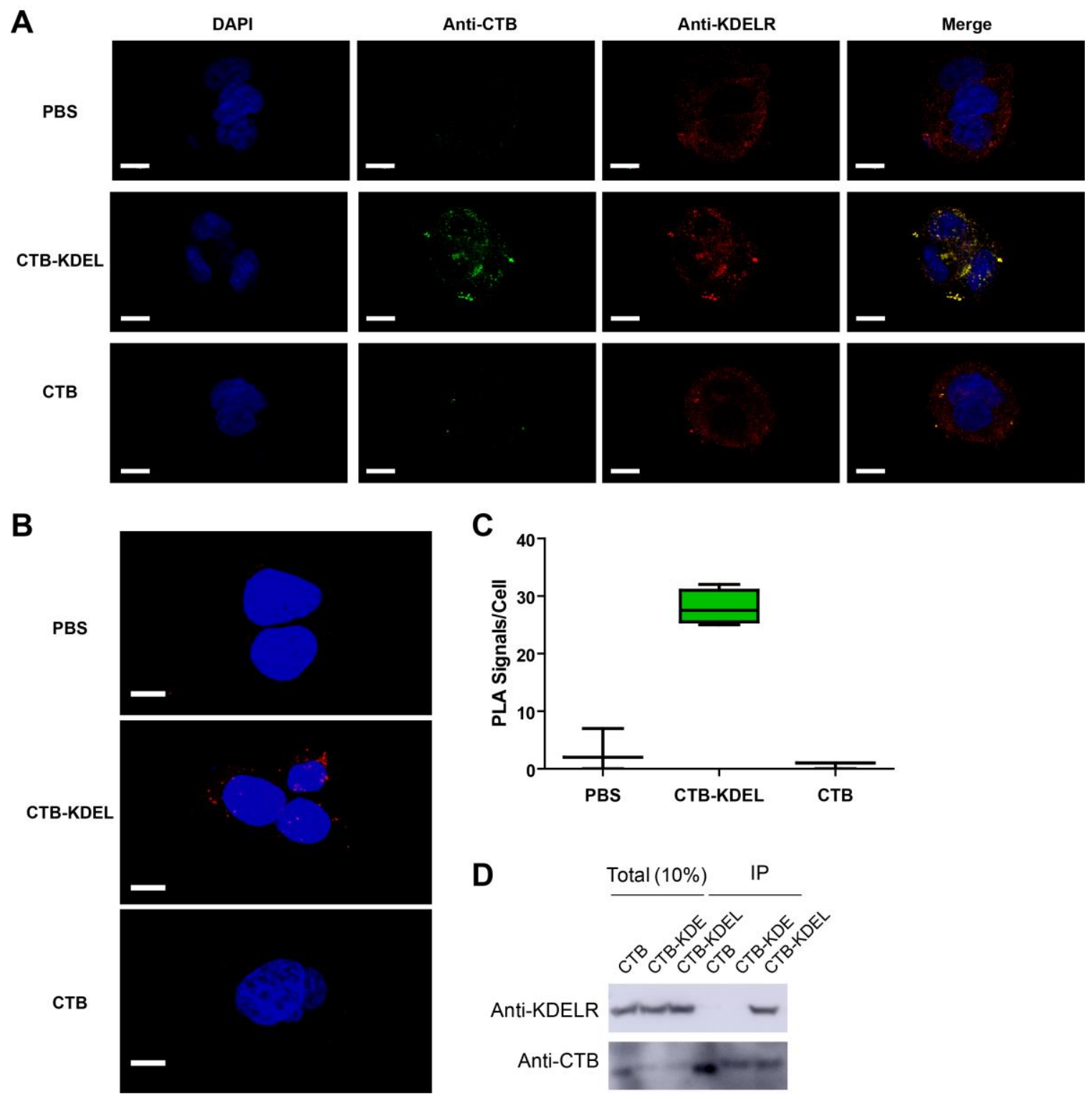

Figure 5. CTB-KDEL binds to the KDEL receptor (KDELR). (A)

Immunofluorescence analysis of CTB-KDEL or CTB intracellular localization within the ER. Caco2 Cells were treated with $1 \mu \mathrm{M}$ of CTB or CTB-KDEL, or a vehicle control (PBS) for $24 \mathrm{~h}$. Cells were fixed/permeabilized and stained with an anti-CTB monoclonal antibody detected by Alexa FluorTM 488 (green), Anti-KDEL polyclonal antibodies (red), and DAPI (blue). (B) Proximity ligation assay (PLA) of CTB-KDEL or CTB protein-protein interaction with KDELR within the ER. Caco2 Cells were treated with 1 $\mu \mathrm{M}$ of CTB or CTB-KDEL, or a vehicle control (PBS) for $24 \mathrm{~h}$. (A, B) Slides were 
analyzed by wide-field fluorescence confocal microscope (60x magnification, Z-stacked images). (C) PLA signals per cell $(\mathrm{n}=3$ ). (D) Co-immunoprecipitation (Co-IP) of KDEL receptor with CTB or CTB-KDEL. Caco2 cell lysates were incubated with $4 \mu \mathrm{g}$ of CTB or CTB-KDEL for $24 \mathrm{~h}$. Ten \% of mixture was presented in total lanes. CTB antibodies and Protein A beads were loaded for immunoprecipitation. After 3 wash steps, beads in sample buffer were boiled at $95^{\circ} \mathrm{C}$ for 10 minutes and then, supernatants were loaded in IP lanes. (D) Performed by Dr. Youngjun Oh

3.2.2 CTB-KDEL Induces an Unfolded Protein Response and IRE1-XBP1 Signaling in Caco2 Cells.

It is well known that accumulation of aberrant proteins in the ER can lead to an unfolded protein response (UPR) and subsequently activate various intracellular signaling pathways [112-114]. Such aberrant proteins would usually be unfolded and misfolded polypeptides of endogenous origin, but adventitious proteins such as CTB may also induce UPR $[54,115]$. There are three branches of UPR known in mammalian cells, including IRE1 (inositol-requiring enzyme 1), PERK (Protein Kinase Related-like ER kinase), and ATF6 (Activating transcription factor $6 ; \alpha$ and $\beta$ isoforms) [116, 117]. Among these, the IRE1-XBP1 pathway has been linked to wound healing and mitigation of DSS colitis [118-120]. Therefore, we tested whether CTB-KDEL induces UPR and IRE1-XBP1 signaling in Caco2 cells. A RT-qPCR analysis showed that, when cells were treated with PBS, CTB or CTB-KDEL for $24 \mathrm{~h}$, all three sensors of the UPR pathway (ATF6, PERK, IRE1), as well as spliced $X B P 1(s X B P 1)$ and $T G F B 1$, were significantly upregulated in CTB-KDEL-treated cells when compared to PBS and/or CTB (Figure 6A). Congruent with the RT-qPCR results, an immunoblot analysis revealed significantly 
increased IRE1 $\alpha$, phosphorylated IRE1 $\alpha$, PERK, and Binding Immunoglobulin Protein (BiP) levels in CTB-KDEL treated cells, but not in PBS, CTB or G33D-CTB-KDELtreated cells (Figure 6B \& C).
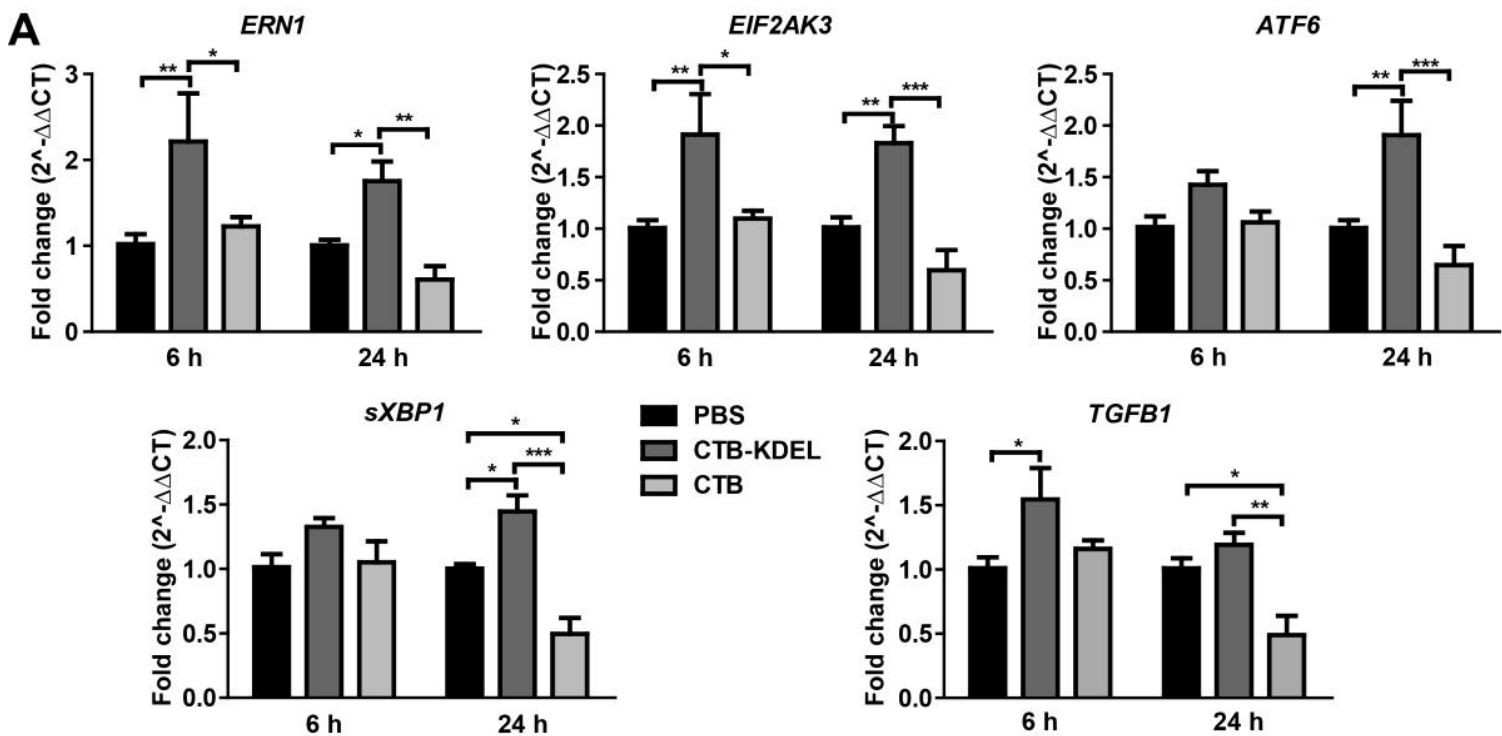

PBS

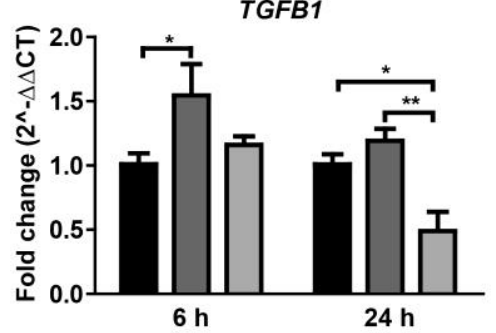

B

C
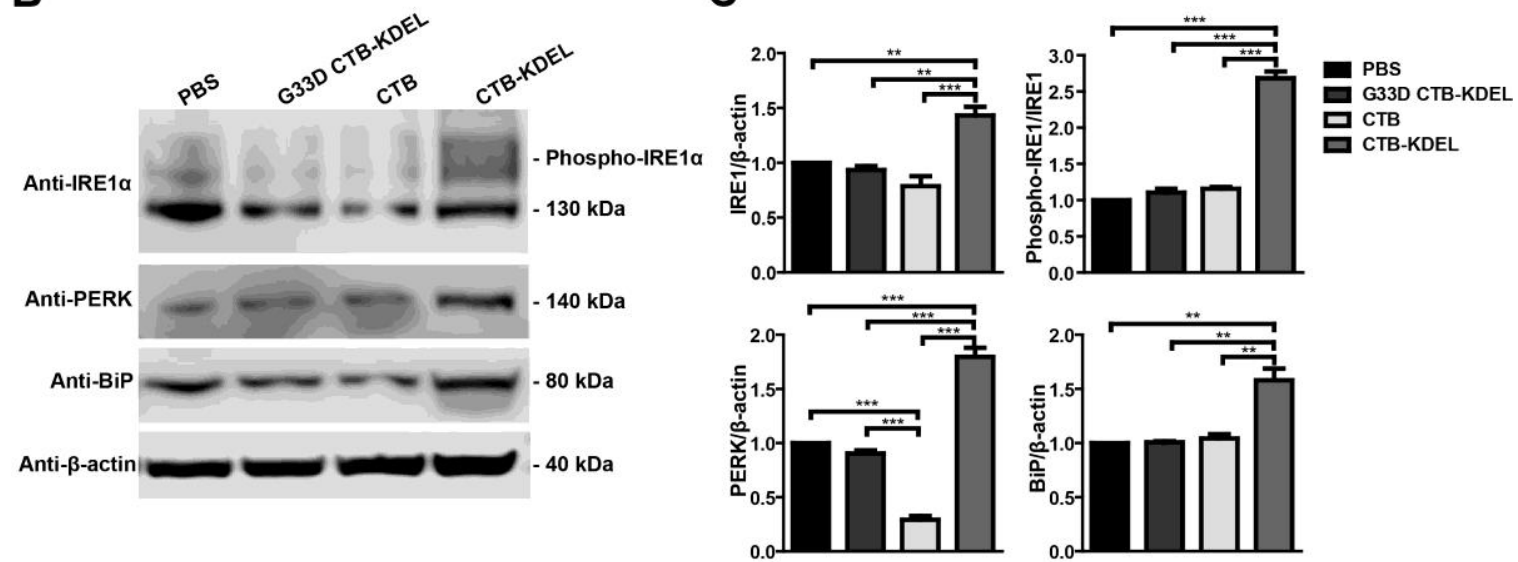

D
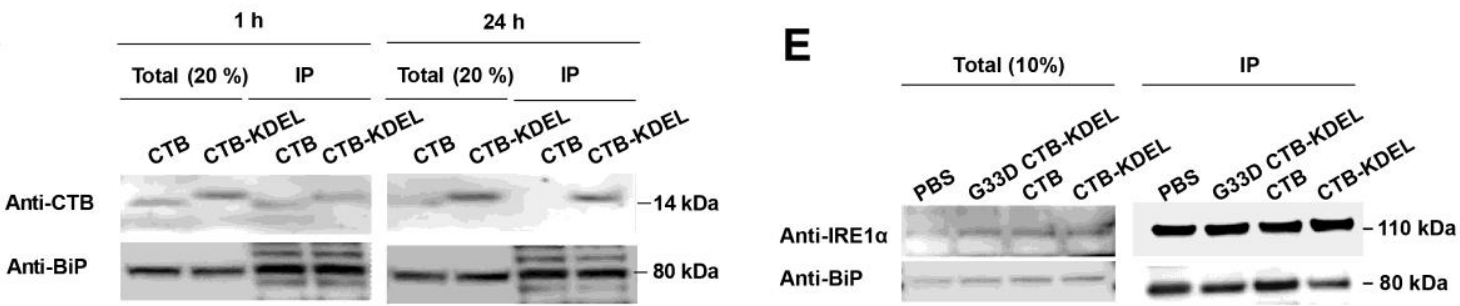

Figure 6. CTB-KDEL induces the unfolded protein response (UPR). Caco2 cells were grown to confluence and scratched. Cells were then incubated with PBS, CTB, 
and/or CTB-KDEL (A) RT-qPCR analysis of UPR gene expression in Caco2 cells $6 \mathrm{~h}$ or $24 \mathrm{~h}$ after treatment. One-way ANOVA with Bonferroni's multiple comparison tests was used to compare all pairs of groups. Mean \pm SEM is shown for each group $(N=4)$. (B) Immunoblot analysis of ER stress/UPR-related proteins. Caco2 cells were incubated for $24 \mathrm{~h}$ with PBS, $1 \mu \mathrm{M}$ of G33D-CTB-KDEL (a non-GM1 binding CTB-KDEL mutant), $\mathrm{CTB}$, or CTB-KDEL. Two-hundred $\mu \mathrm{g}$ of total protein were loaded for immunoblot analysis. $\beta$-Actin was used a loading control. A representative image from three independent analyses is shown. (C) The intensities of western blot bands were measured by Image J program. All graphs showed relative values and error bar means standard deviation ( $\mathrm{n}=3$ ). (D) Co-immunoprecipitation of BiP with CTB or CTB-KDEL. For 1 hour or 24 hours, Caco 2 cells were incubated with $1 \mu \mathrm{M}$ CTB and $1 \mu \mathrm{M}$ CTB-KDEL. 20 $\%$ of intracellular extracts were loaded in total lanes (Total $20 \%$ ). Intracellular extracts were incubated with $4 \mu \mathrm{g}$ of $\mathrm{BiP}$ antibodies for $2 \mathrm{~h}$ at $4{ }^{\circ} \mathrm{C}$. Ten $\mu 1$ of Protein A beads were employed to precipitate the binding complex of BiP. After washing, samples were loaded in immunoprecipitation lanes (IP). Anti-CTB antibodies showed CTB and CTBKDEL proteins in western blot (Anti-CTB) and BiP antibodies detected BiP proteins (Anti-BiP). (E) Co-immunoprecipitation of IRE1 $\alpha$ with BiP. For 24 hours, caco-2 cells were incubated with PBS buffer (PBS), $1 \mu$ M G33D CTB mutants (G33D CTB-KDEL), $1 \mu \mathrm{M} \mathrm{CTB}$, and $1 \mu \mathrm{M}$ CTB-KDEL. $10 \%$ of intracellular extracts were loaded in total lanes (Total $10 \%$ ). Intracellular extracts were incubated with $4 \mu \mathrm{g}$ of IRE1 $\alpha$ antibodies for $2 \mathrm{~h}$ at $4{ }^{\circ} \mathrm{C}$. Ten $\mu 1$ of Protein A beads were employed to precipitate the binding complex of IRE1 $\alpha$. After washing, samples were loaded in immunoprecipitation lanes (IP). Anti-IRE1 $\alpha$ antibodies showed IRE1 $\alpha$ proteins in western blot (Anti-IRE1 $\alpha$ ) and $\mathrm{BiP}$ antibodies detected BiP proteins (Anti-BiP). (B-D) Performed by Dr. Youngjun Oh 
The onset of UPR is preceded by the dissociation of BiP from the UPR sensors (ATF6, PERK, IRE1), which is caused by the attraction of the molecular chaperone by aberrant proteins in the ER $[121,122]$. Accordingly, we examined the binding of BiP with CTB-KDEL in Caco2 cells. A co-immunoprecipitation assay using an anti-BiP antibody revealed that, when the cells were incubated with CTB or CTB-KDEL for $1 \mathrm{~h}$, both proteins formed a complex with BiP (Figure 6D). However, after $24 \mathrm{~h}$ incubation, only CTB-KDEL was shown to interact with the ER chaperone (Figure 6D). These results indicate that the $\mathrm{CTB}$ domain has the capacity to interact with $\mathrm{BiP}$ and that the retention of CTB-KDEL in the ER (by the KDELR) prolonged their interaction. To confirm this observation, we monitored the amounts of IRE1-BiP complex in a coimmunoprecipitation assay using an anti-IRE1 $\alpha$ antibody after incubating Caco 2 cells with G33D-CTB-KDEL, CTB or CTB-KDEL, or PBS (Figure 6E). The results revealed that the association between $\mathrm{BiP}$ and IRE1 $\alpha$ was significantly reduced by CTB-KDEL, but not by PBS, G33D-CTB-KDEL or CTB. 
A

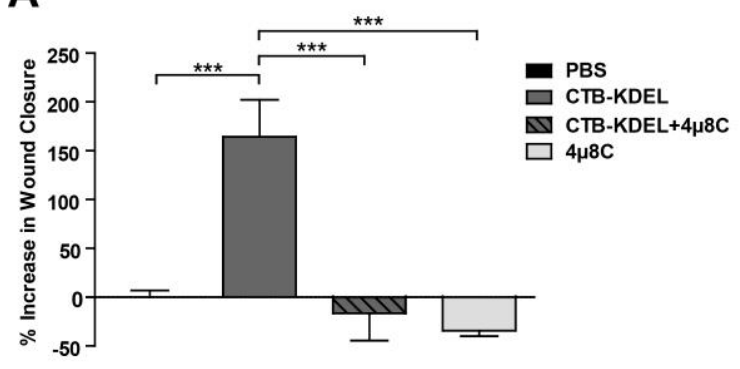

C

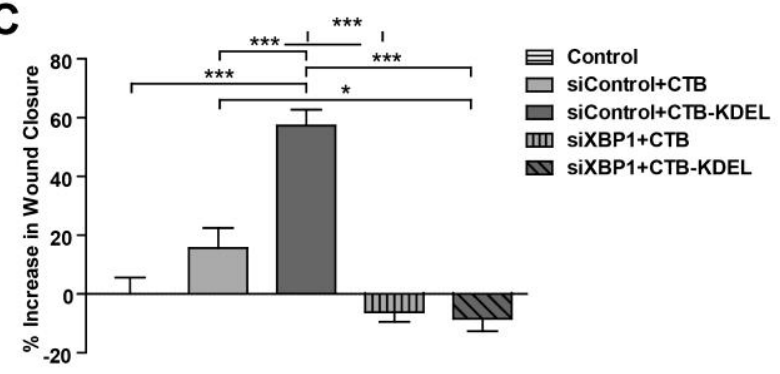

B
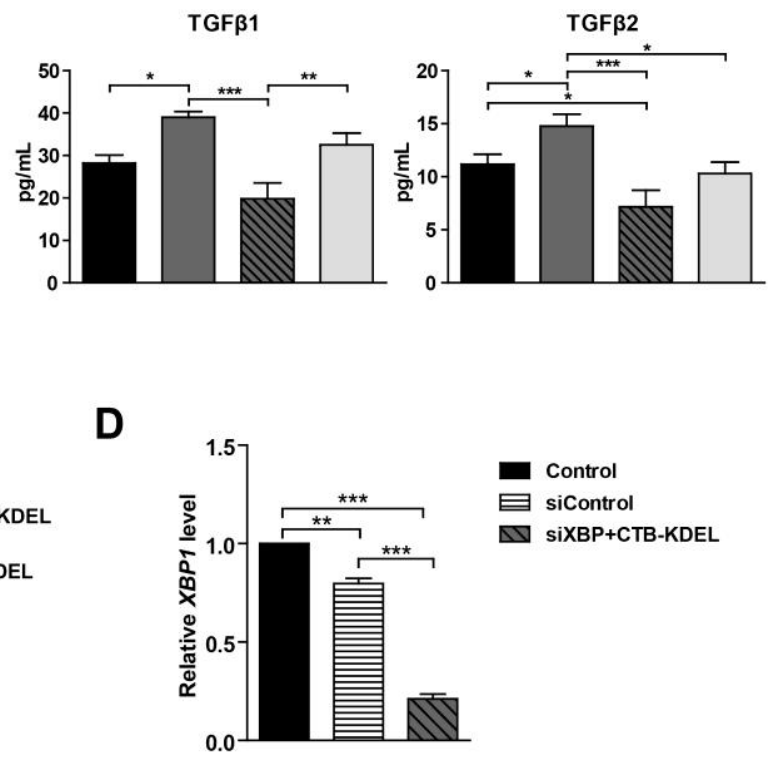

Figure 7. CTB-KDEL's epithelial wound healing activity is mediated through IRE1-

XBP1 signaling. (A) Caco2 cells were grown to confluence and scratched with a pipette tip. Cells were then incubated with PBS, CTB, CTB-KDEL, and/or $4 \mu 8 \mathrm{C}$. Wound closure over $24 \mathrm{~h}$ was analyzed. Mean percentage closure was determined by Image $\mathbf{J}$ software ( $\mathrm{n}$ =4). (B) TGF $\beta$ concentrations in Caco2 cell supernatants from wound healing assay in (A). Mean \pm SEM is shown for each group $(\mathrm{N}=4)$. (C) Impact of XBP1-knockdown on CTB-KDEL's wound healing activity. Caco2 cells $(n=6)$ were transfected with control siRNAs (siCon) or XBP1-targeted siRNAs (siXBP1). After $72 \mathrm{~h}$, cells were scratched and treated with $1 \mu \mathrm{M}$ of CTB or CTB-KDEL. The wound closure was measured at $24 \mathrm{~h}$. (D) The transcript levels of XBP1 were measured by qRT-PCR, using $18 \mathrm{~S}$ rRNA as a reference. $(\mathrm{A}-\mathrm{D}) *, * *, * * * \mathrm{P}<0.05,0.01$ and 0.001 : One-way ANOVA with Bonferroni's multiple comparison tests. (C-D) Performed by Dr. Youngjun Oh

Collectively, the above findings strongly suggest that CTB-KDEL's prolonged residence in the ER induces a UPR in Caco2 cells, which may be in part due to the 
protein's interaction with the ER chaperone BiP. To test the impact of the UPR on CTBKDEL's wound healing effect, we treated Caco2 cells with CTB-KDEL in the presence or absence of $4 \mu 8 \mathrm{C}$, an inhibitor of IRE1-mediated XBP1 splicing (and hence inhibits the activation IRE1-XBP1 signaling pathway [123]), after scratching the cell monolayer. The analysis revealed that $4 \mu 8 \mathrm{C}$ completely blocked CTB-KDEL's cell migration effects; whereas CTB-KDEL treatment significantly enhanced wound closure, CTBKDEL $+4 \mu 8 \mathrm{C}$ and $4 \mu 8 \mathrm{C}$ treated groups showed no effect compared to the PBS control group (Figure 7A). Furthermore, as shown in Figure 7B, CTB-KDEL treatment significantly increased TGF $\beta 1$ and TGF $\beta 2$ levels when compared to PBS, CTB$\mathrm{KDEL}+4 \mu 8 \mathrm{C}$ or $4 \mu 8 \mathrm{C}$ treatment, indicating that CTB-KDEL increases TGF $\beta 1$ and TGFß2 levels and subsequent cell migration via the IRE1-XBP1 signaling pathway. These results are further supported by an XBP1 knock-down experiment, where Caco2 cells were transfected with control siRNAs (siCon) or XBP1-targeted siRNAs (siXBP1) and then cells were scratched and treated with CTB-KDEL or CTB. The results clearly showed that siXBP1 treatment significantly reduced CTB-KDEL-induced upregulation of $\mathrm{XBP1}$ levels as well as cell migration activity (Figure 7C, D).

\subsubsection{CTB-KDEL Induces A UPR in Primary Colon Epithelial Cells and Facilitates} Colonic Wound Healing After DSS-Induced Acute Colitis in Mice 

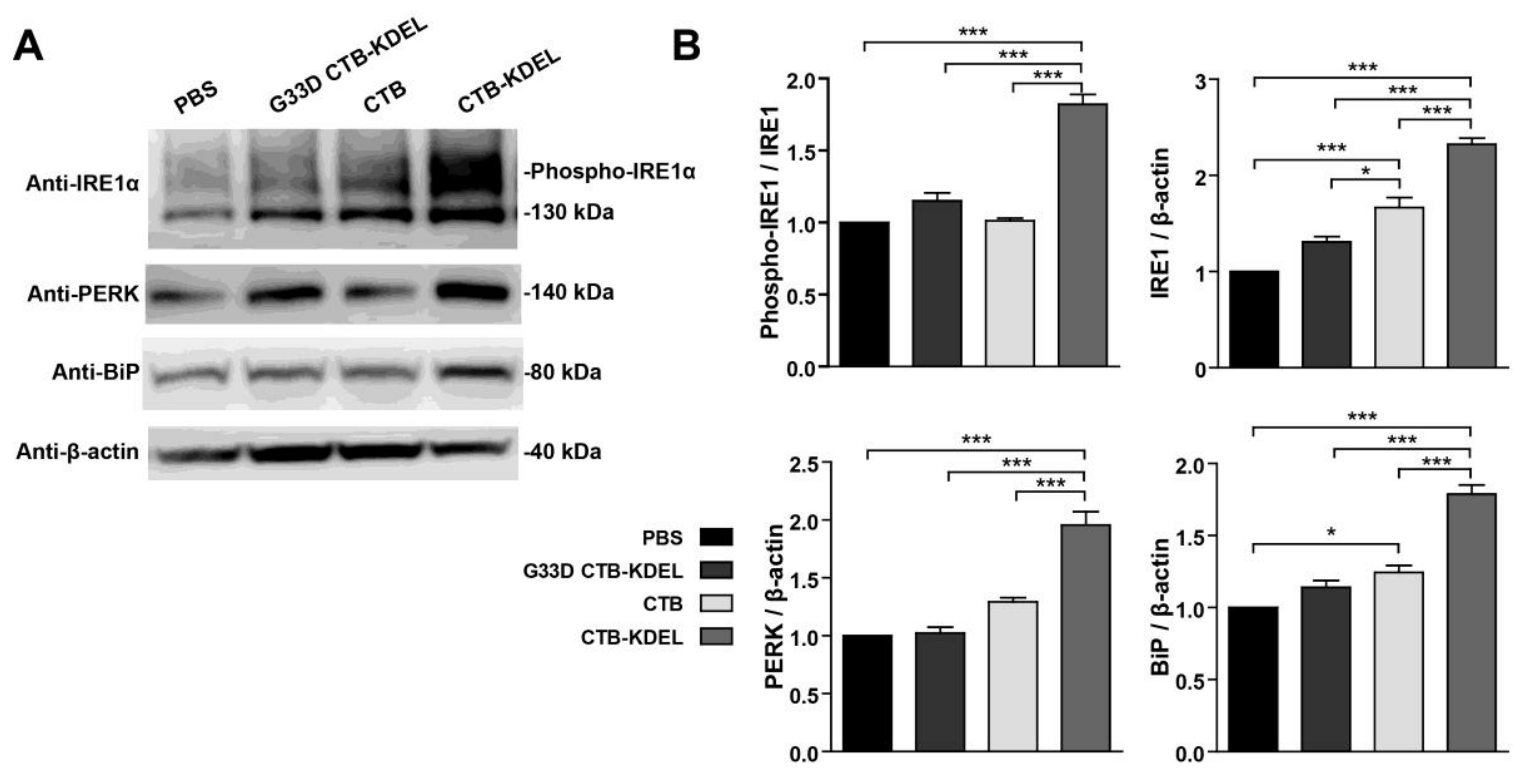

Figure 8. Immunoblot analysis of UPR-related proteins. Mouse primary colon epithelial cells were incubated for $24 \mathrm{~h}$ with PBS, or $1 \mu \mathrm{M}$ of G33D-CTB-KDEL (a nonGM1 binding CTB-KDEL mutant), CTB or CTB-KDEL. (A) Two-hundred $\mu$ g of total protein were loaded for immunoblot analysis. $\beta$-Actin was used a loading control. A representative image from three independent analyses is shown. (B) The band intensities of western blot bands were measured by Image J. All graphs show relative values and bars represent mean \pm SEM (n=3). (A-B) Performed by Dr. Youngjun Oh

To determine if the above-described mechanism by which CTB-KDEL induces cell migration in vitro is applicable to intestinal wound healing in vivo, we isolated and treated mouse primary colon epithelial cells with PBS, CTB-KDEL, CTB or G33D-CTBKDEL for $24 \mathrm{~h}$. An immunoblot analysis revealed that CTB-KDEL significantly increased IRE1 $\alpha$, phosphorylated IRE1 $\alpha$ (p-IRE1 $\alpha$ ), PERK and BiP levels compared to all other treatment groups (Figure 8A, B). Albeit to a lesser extent, CTB also significantly increased the levels of IRE1 $\alpha$ and BiP when compared to PBS; however, 
CTB did not increase the p-IRE1 $\alpha$ level. Next, we employed a mouse acute DSS colitis model to compare the wound healing efficacies of CTB-KDEL and CTB. Female C57bl/6 mice were given one dose of PBS, $3 \mu \mathrm{g}$ CTB-KDEL or $3 \mu \mathrm{g}$ CTB via gavage after a 7-day 3\% DSS exposure period (Day 7), at which the onset of colonic epithelial damage had taken place [97]. As shown in Figure 8A, CTB-KDEL-dosed mice showed a significantly more rapid recovery from DSS-induced weight loss than PBS and CTBdosed groups. This trend was noted as early as 3 days post administration (Day 10) through Day 14 at which the mice were sacrificed. The recovery in body weight was accompanied by a significant protection from colon shrinkage as well as significant decrease in disease activity index (DAI) and histopathological crypt scores at sacrifice in CTB-KDEL-administered animals (Figure 8B). Furthermore, a complete blood count analysis of terminal blood samples (collected on Day 14) showed that CTB-KDEL treatment significantly reduced the DSS-induced escalation of monocyte, basophil and eosinophil levels compared to PBS-dosed mice. Neutrophil levels were significantly higher in PBS and CTB-dosed mice compared to those of the healthy control animals, but not in CTB-KDEL-dosed animals (Figure 8C). Similar results were also obtained in male C57b1/6 mice, in which CTB-KDEL, but not CTB, significantly facilitated recovery from acute DSS colitis on the basis of DAI, colon length and crypt scores, suggesting that there is no sex-related difference in the therapeutic effects of CTB-KDEL (Figure 8D). Additional histopathological examination on hematoxylin and eosin (H\&E)-stained distal colon tissues of the female mice revealed that CTB-KDEL treatment protected from ulceration, inflammation and loss of colonic epithelial surface and crypts, in contrast to PBS and CTB treatment (Figure 10A). Moreover, immunohistochemistry (IHC) staining for an epithelial marker, E-cadherin [124, 125], on the same tissues used for 
histopathological examination, clearly revealed that CTB-KDEL-treated mice had an epithelial surface that has recovered (or maintained) near-normal thickness, with ongoing crypt regeneration noted throughout the tissue (Figure 10A). Consistent with these findings, a RT-qPCR analysis revealed that CTB-KDEL administration significantly upregulated multiple genes associated with epithelial repair, including $C d h 1, W n t 5 a$, and Col5a3 (Figure 10B) [126-129]. Collectively, these results demonstrate CTB-KDEL's unique ability to mitigate DSS-colitis and facilitate epithelial repair in mouse colons. Given that CTB-KDEL activated an UPR in primary mouse colon epithelial cells (Figure 8), the enhanced epithelial repair was likely mediated via the UPR, or specifically the IRE1 pathway, as demonstrated in the Caco2 model (Figure 7). 
A

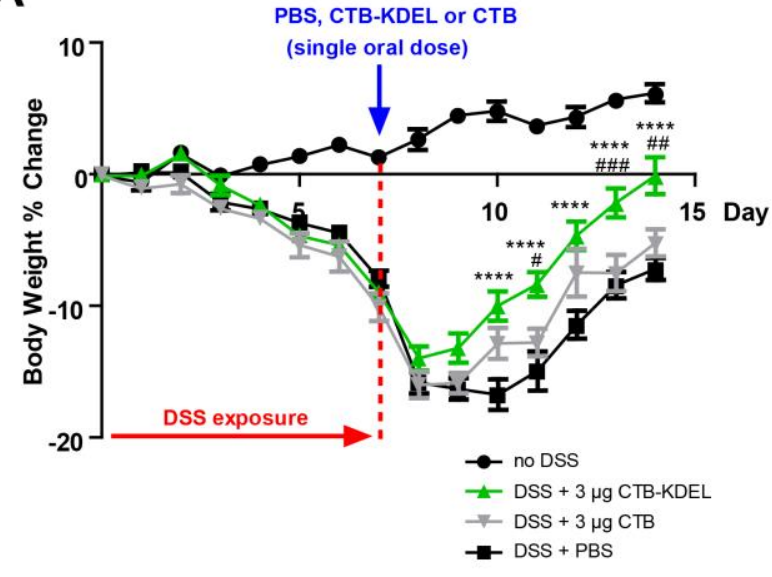

C

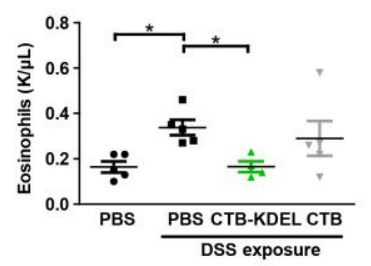

D
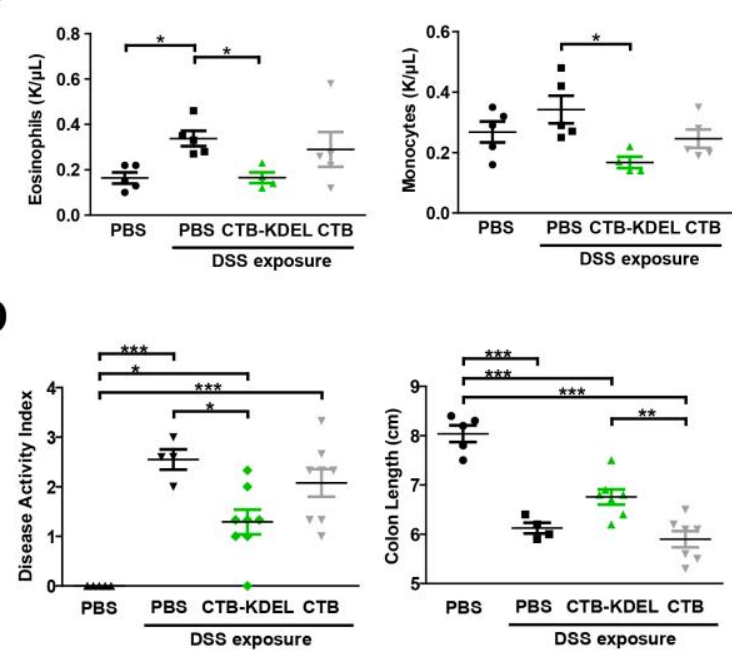

B
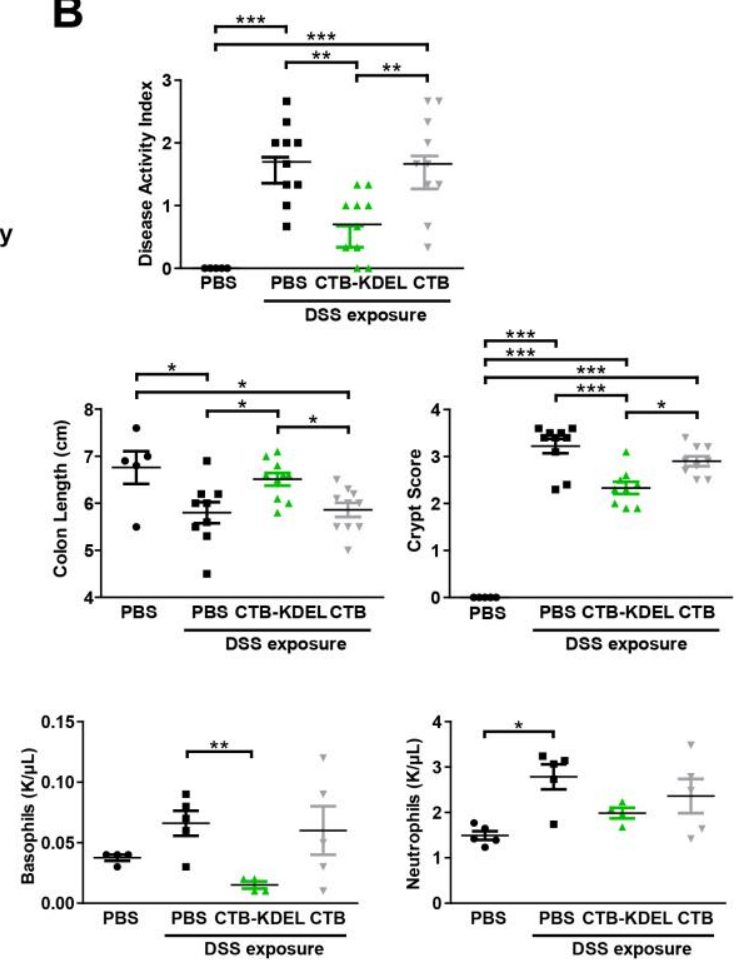

Figure 9. Effects of orally administered CTB or CTB-KDEL in an acute DSS-colitis

model. Mice (female and male C57BL/6J, 9-week old) were exposed to 3\% DSS for seven days and orally administered with PBS, CTB, or CTB-KDEL on the 7 seventh day. Colon tissues were isolated after a 7-day recovery for analyses. Mean \pm s.e.m. is shown for each group. $\mathrm{N}=10$ Animals per group. (A) Female mice percent change of body weights. Animals were weighed daily and just prior to the initiation of DSS exposure. Percent change was based on the initial body weight. *P $<0.05$ between DSS-exposed, CTB-KDEL and PBS-administered groups, "P $<0.05$ between DSS-exposed, CTBKDEL and CTB-administered groups; two-way ANOVA with Bonferroni's multiple 
comparison tests. (B) Top-Right: Female mice disease activity index (DAI) scores calculated from body weight loss, fecal consistency and occult blood scores at the time of sacrifice. Bottom-left: Colon length measured at sacrifice. Bottom right: Colon crypt scoring from paraffin embedded tissue sections were scored after staining with Hematoxylin and Eosin (H\&E). Scoring was based on 0-4 scale. Top-right: Colon length. (C) Female mice complete blood count analysis performed at the time of sacrifice. Mean \pm SEM is shown for each group ( $N=5$ ). (D) Male mice (male C57BL/6J, 9-week old) DAI scores, colon length and colon crypt score. Mean \pm s.e.m. is shown for each group $\mathrm{N}$ $=10$. Bonferroni's multiple comparison tests was used to compare all pairs of groups (A, $\mathrm{B}, \mathrm{C}, \mathrm{D})$. Significantly different pairs are highlighted with asterisks $(* \mathrm{P}<0.05, * * \mathrm{P}<$ 0.01 , and $* * * \mathrm{P}<0.001)$. 
A
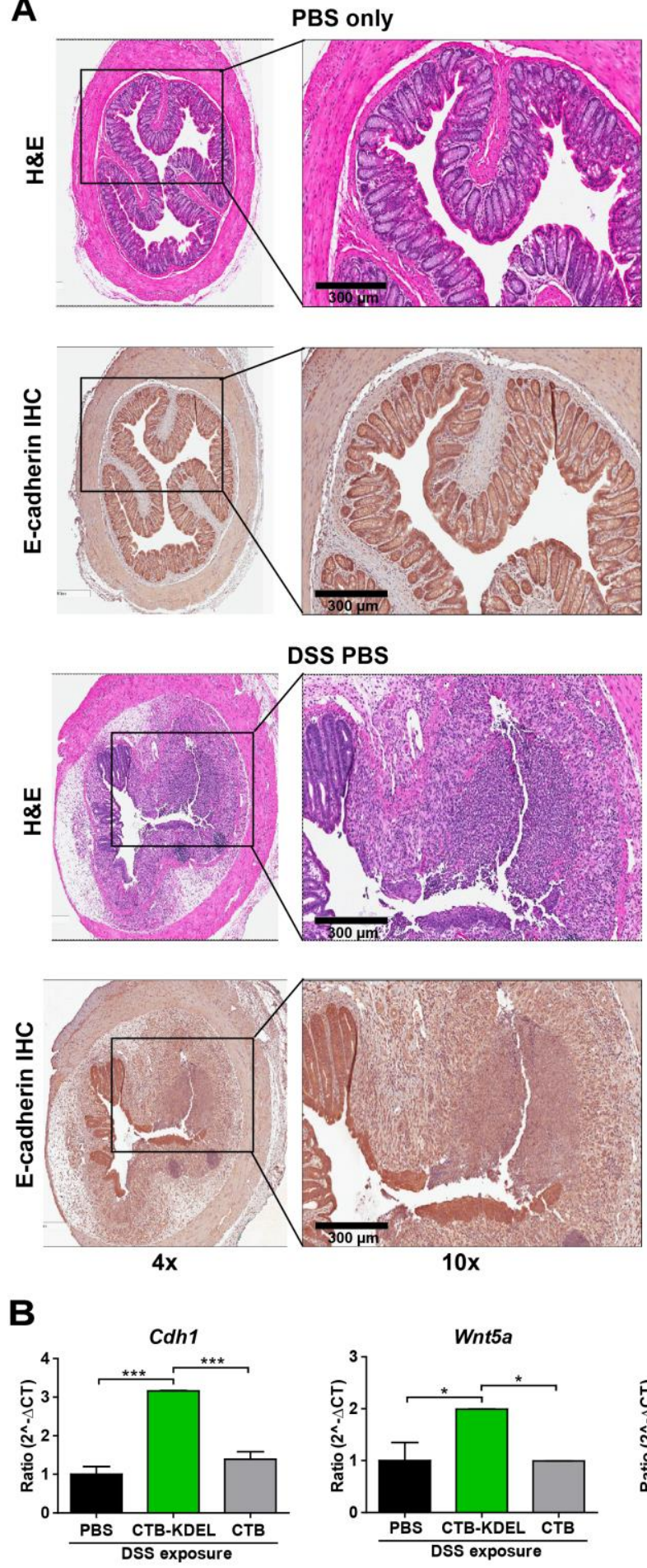

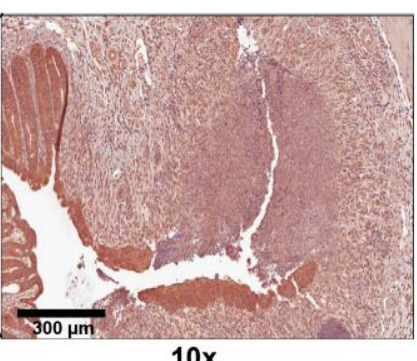

$10 x$

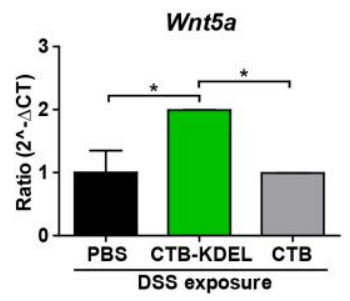

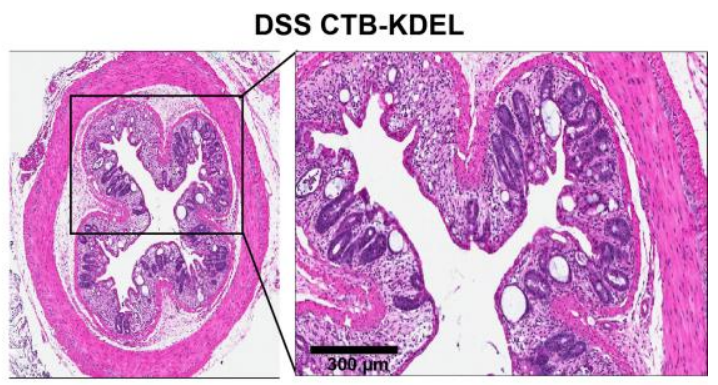
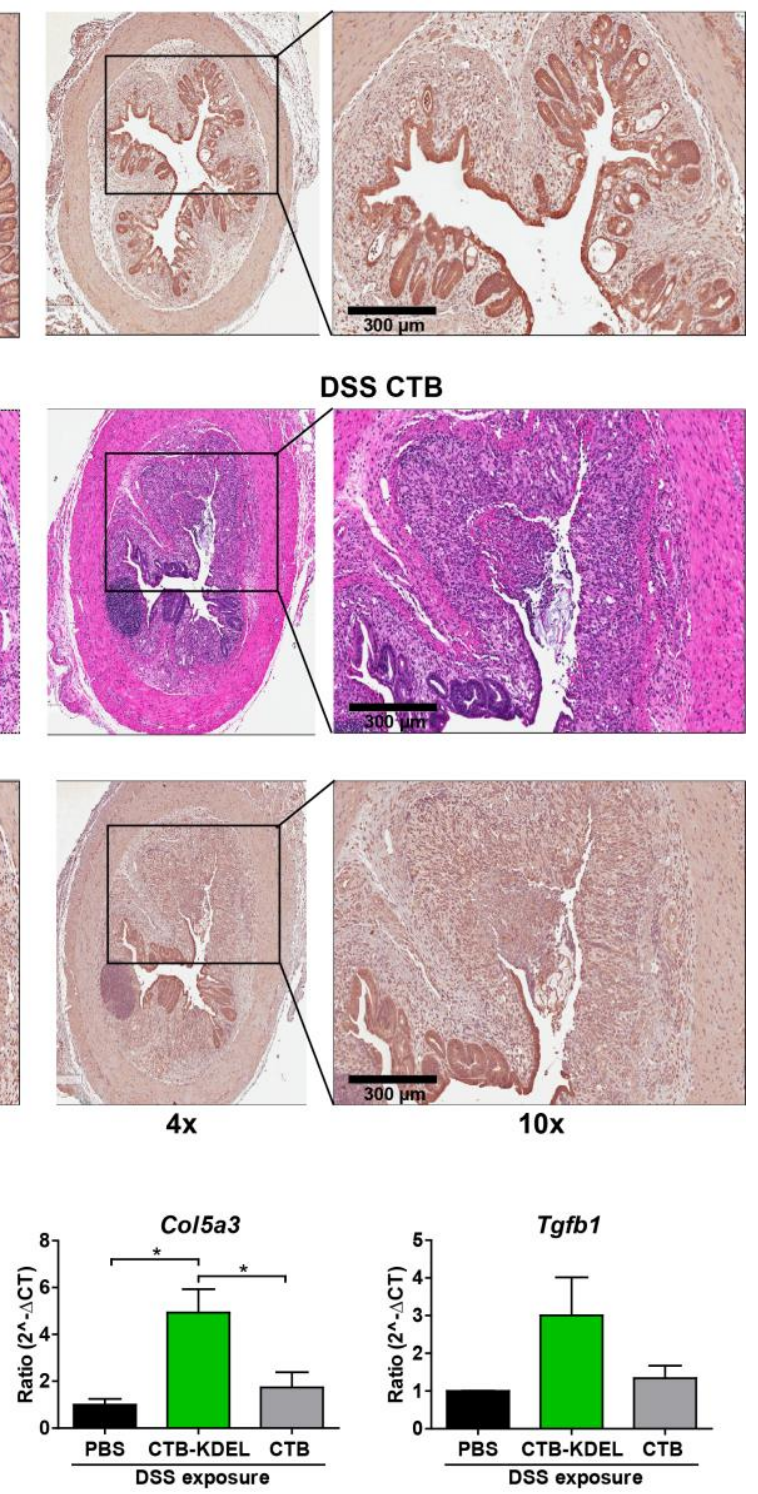

Figure 10. CTB-KDEL and CTB effects on mouse colon histological alterations

induce by DSS-colitis. CTB-KDEL treatment protected mice from ulceration,

inflammation and loss of colonic epithelial surface and crypts. (A) Top: representative $4 x$ 
(left) and 10x (right) photomicrographs of H\&E stained distal colon tissues from each treatment group. Bottom: Immunohistochemistry (IHC) staining of E-cadherin positive cells in distal colon tissue, representative photomicrographs are shown. (B) qRT-PCR analysis of cytokine gene expression in mouse colon tissue. Mean \pm SEM is shown for each group $(\mathrm{N}=4)$.

\subsection{Discussion}

Here we aimed to delineate the mechanism by which CTB-KDEL exhibits unique colon epithelial wound repair activity that has not been previously observed with CTB. Our data reported herein demonstrated that it is ascribed to the C-terminal ER retention motif, which was added initially to improve the yield of the cholera vaccine antigen by means of accumulation in the ER of host plant cells upon transient overexpression [107]. However, the KDELR is highly conserved among eukaryotic organisms, including plant and mammalian cells, signifying that CTB-KDEL could interact with the receptor within human IECs. Once bound to KDEL-containing proteins, the receptor transports proteins from the Golgi apparatus to the ER [52, 54, 102]. It has been previously shown that KDELR-mediated retention of proteins in the ER can lead to a UPR in human cells [130, 131]. Meanwhile, a UPR has been linked to TGF $\beta$ activation in hepatocytes, skin fibroblasts and lung epithelial cells [132-134]. Consequently, it is deemed reasonable that CTB-KDEL induces TGF $\beta$ signaling via a UPR in IECs, leading to the new function of mucosal wound healing in colitis models. Our data provide multiple lines of evidence to support the above notion. First, a variant of CTB-KDEL that lacks the terminal leucine residue (CTB-KDE) failed to induce cell migration in Caco2 cells (Figure 2). Second, CTB-KDEL colocalized with KDELR and remained longer than CTB within the ER of 
Caco2 cells (Figures 4 and 5). Previous studies investigating the intracellular trafficking of CT and CTB have shown that CTB's retrograde transportation is triggered upon binding to cell-surface GM1-ganglioside, which allows the proteins to reach deep endomembrane compartments and the ER [54, 135]. As shown in Figures 4 and 5, both CTB and CTB-KDEL were found in the ER, however, the latter was more consistently retained and localized within the ER, likely due to its capacity to interact with the KDEL receptor. Third, CTB-KDEL, but not CTB, increased the levels of UPR sensors by $1.5-$ 2-fold in Caco2 and mouse primary colon epithelial cells (Figures 6 and 8). Finally, genetic and chemical inhibition of IRE1-XBP1 signaling completely abolished the TGF $\beta$ upregulation and cell migration effects of CTB-KDEL in Caco2 cells (Figure 7). Combined with our previous data showing that GM1 binding is essential for CTBKDEL's wound healing effect in Caco2 cells [50], these data strongly suggest that the Cterminal KDEL sequence rendered CTB-KDEL with a unique new wound healing activity in the colon epithelial cells.

Accumulation of aberrant proteins in the ER promotes the dissociation of BiP from the three main ER transmembrane sensors, IRE1, PERK and ATF6, which triggers their dimerization and phosphorylation for the activation of sensor signal transduction leading to the UPR [116, 136, 137]. Our data demonstrated that CTB-KDEL indeed interacted with $\mathrm{BiP}$, which in turn induced the dissociation of $\mathrm{BiP}$ from IRE1 complex (Figure 6). Therefore, it is likely that the binding of CTB-KDEL to BiP plays a role in the initiation of UPR seen with CTB-KDEL treatment. IRE1 represents the most evolutionarily conserved branch of the UPR that is made up of two isoforms, IRE1 $\alpha$ and IRE1 $\beta$, of which IRE1 $\beta$ is unique to the epithelium of the digestive and respiratory tract [138]. IRE1-XBP1 signaling has been linked to TGF $\beta$ activation, wound healing, colon 
epithelial cell prosurvival signaling, and protection from DSS-induced colitis, as mentioned previously $[118,120,132,133,139]$. In this regard, CTB-KDEL significantly increased IRE1 $\alpha$ expression $6 \mathrm{~h}$ after treatment and all 3 arms of UPR sensors (ATF6, PERK, and IREI), as well as the IRE1 signal transducer XBP1, $24 \mathrm{~h}$ after treatment (Figure 6). Furthermore, inhibition of IRE1 signaling by the chemical inhibitor $4 \mu 8 \mathrm{C}$ completely blocked the wound healing activity of CTB-KDEL as well as CTB-KDELmediated TGF $\beta 1$ and TGF $\beta 2$ induction (Figure 7) indicating that CTB-KDEL's wound healing activity is mediated through IRE1 signal transduction. Of note, UPR activation is closely linked to ER stress and apoptotic pathways [138]. However, we did not observe any cytotoxic response to CTB-KDEL up to $10 \mu \mathrm{M}(0.61 \mathrm{mg} / \mathrm{mL})$ in Caco2 cells (Figure 2), suggesting that the protein did not overstimulate UPR beyond the threshold for a cell death response. In our preliminary study using polarized T84 cell monolayers, we found that the levels of UPR marker genes induced by CTB-KDEL were significantly lower than the well-known UPR inducer thapsigargin (data not shown). It is possible that the amount of cell-surface GM1 ganglioside was a limiting factor, whereby the receptor became saturated before the concentration of CTB-KDEL reached a point that overload the ER. Further investigation is necessary to understand CTB-KDEL's impacts on UPR, ER stress and survival in epithelial cells.

In the mouse DSS acute colitis model, a single oral administration of as little as 3 $\mu \mathrm{g}$ of CTB-KDEL significantly enhanced recovery from colitis (Figures 9 and 10). The therapeutic effects were characterized by enhanced epithelial regeneration, demonstrated by E-cadherin IHC and upregulated $C d h 1$ and $W n t 5 a$ levels, suggesting that the rapid resolution of epithelial injury is the primary mechanism of CTB-KDEL's efficacy in this model. It should be noted that CTB failed to show such effects and was essentially futile 
in this model. This is in sharp contrast to previous findings by Boirivant et al., which showed that oral administration of CTB can resolve TNBS (2,4,6-trinitrobenzenesulfonic acid)-induced colitis via Th1 cell inhibition [70, 77]. Although a direct comparison cannot be made due to two different colitis models, the discrepancy in CTB's efficacies could be in part explained by the different dose amounts and timings employed in those two studies; whereas Boirivant et al. administered 4 daily doses of $100 \mu \mathrm{g}$ CTB immediately after the administration of TNBS [70,77], we dosed one $3 \mu \mathrm{g}$ dose of CTBKDEL or CTB at the end of DSS exposure. This points to the possibility that the low dose of CTB was not sufficient to show a therapeutic effect in the DSS model. Conversely, whether CTB-KDEL at high dose levels exhibits T cell-inhibitory effects like CTB remains to be determined.

In DSS-induced colitis model, the epithelium receives a barrage of mucosal insults, both physical and chemical that result in the loss of the epithelial barrier and damage to the mucosa [95]. Thus, the initial step in injury repair occurs through a rapid migration response of the epithelial sheet (termed restitution) [140, 141]. The process occurs independently of proliferation and results in depolarization of IECs surrounding the wounded area [141]. This depolarization leads to an epithelial-to-mesenchymal transition (EMT) where cells adopt a migratory phenotype induced by increased TGF $\beta$ levels $[142,143]$. Previously, we showed that CTB-KDEL induces multiple TGF $\beta$ dependent EMT pathways after treating mice with CTB-KDEL [50]. However, the TGF $\beta$ expression level in the CTB-KDEL-dosed animals was not significantly high, although upregulated 3-fold, at the time of sacrifice, suggesting that the cytokine had likely passed its peak expression point. In fact, we observed a significant upregulation of Serpine1, an inhibitor of TGF $\beta$, in the CTB-KDEL-dosed group (data not shown). Additionally, we 
detected upregulated $C d h 1$ gene in CTB-KDEL-treated mice (Figure 10), which is indicative of a late-phase wound healing involving epithelial proliferation and maturation $[126,127,129,144,145]$. E-cadherin expression is inversely correlated with TGF $\beta$ levels due to TGF $\beta$-induced class switching of E-cadherin to N-cadherin which depolarizes cells and allows them to become of migratory phenotype [143, 145, 146]. Thus, the elevated $C d h 1$ levels and strong E-cadherin positive epithelial cell staining that lined the mucosa of CTB-KDEL-treated mice indicate the repolarization of the epithelial cells, increased tightening of mucosal barrier and improved mucosal barrier integrity, which are critical steps during epithelial repair [145]. In addition to EMT, TGF $\beta 1$ stimulates and increases expression of WNT5a [144]. WNT5a has been shown to induce new crypt formation and re-establish epithelial homeostasis after injury [144]. The H\&E-stained tissue sections clearly revealed the regeneration and formation of new crypts in the CTBKDEL-dosed group, corroborating the function of upregulated Wnt5a expression in the colon mucosa. Taken together, these data strongly support the notion that oral administration of CTB-KDEL can facilitate colon epithelial restitution and wound healing, at least in the conditions tested in the acute DSS colitis model.

In conclusion, the data herein reveal that CTB-KDEL exhibits unique colon mucosal would healing effects that are mediated by its localization in the ER via KDELR and subsequent activation of a UPR and TGF $\beta$ signaling in colon epithelial cells. Further investigation of the role of the UPR in TGF $\beta$ activation in colon epithelial cells may shed light on the mechanistic link to epithelial restitution and repair, facilitating the development of a new agent for the treatment of inflammatory diseases of the mucosa. 


\section{CHAPTER 4: REPEATED ORAL ADMINISTRATION OF A KDEL-TAGGED RECOMBINANT CHOLERA TOXIN B SUBUNIT EFFECTIVELY MITIGATES DSS COLITIS DESPITE A ROBUST IMMUNOGENIC RESPONSE ${ }^{4}$}

\subsection{Introduction}

The principal virulence factor of Vibrio cholerae is cholera toxin (CT), which is comprised of a toxic A subunit and a non-toxic homopentameric B subunit (CTB). Recently, we have shown that a recombinant variant of CTB containing a KDEL endoplasmic reticulum (ER) retention motif (CTB-KDEL) has the ability to induce mucosal healing, facilitate colon epithelial wound repair, and enhance recovery from an acute dextran sulfate sodium (DSS) colitis model in mice [50, 147]. These effects were attributed to the addition of the C-terminal ER retention motif, KDEL, to CTB. The KDEL sequence enabled CTB-KDEL to bind to the KDEL receptor and localize within the ER in the Caco2 human colon epithelial cell line. Upon ER localization, CTB-KDEL induced an unfolded protein response (UPR) and subsequent TGF $\beta$ signaling. In particular, the inositol-requiring enzyme 1 (IRE1)/X-box binding protein 1 (XBP1) arm of the UPR was indispensable for wound healing activity [147]. These findings were

\footnotetext{
${ }^{4}$ Modified from: Toxins (Bacterial Enterotoxins). 2019 Nov 18; 11(12), 678. https://doi.org/10.3390/toxins11120678
} 
corroborated in primary mouse colon epithelial cells, where CTB-KDEL induced an UPR, while CTB and the non-GM1 binding mutant G33D-CTB-KDEL failed to exhibit such an effect. Moreover, in an ex vivo experiment using colectomy tissue from inflammatory bowel disease (IBD) patients, CTB-KDEL, but not CTB, induced an UPR, upregulated wound healing pathways, and maintained viable crypts [147]. These findings provide implications for the potential use of CTB-KDEL in the treatment of IBD.

It is estimated that $1.3 \%$ of US adults (3.1 million) suffer from IBD, which consists of two main classes, ulcerative colitis (UC) and Crohn's disease (CD). IBD is characterized by chronic periods of remission and relapse [148]. UC mainly affects the mucosa in the rectum and colon, whereas the inflammation in CD affects all layers of the bowel wall including the muscularis and serosa. Moreover, unlike UC, inflammation in CD can occur at any part of the gastrointestinal (GI) tract [5]. Multiple studies using immunosuppressive agents have led to a general consensus that healing of the mucosal layer (i.e., mucosal healing) is the most important treatment goal of IBD $[13,14,19]$. In particular, the most critical component of the mucosal healing in UC, which does not usually involve transmural inflammation, may be epithelial healing/restitution [19]; an effect that could be attained by oral administration of CTB-KDEL. Currently, treatment strategies for UC aim to blunt the inflammatory response and establish remission by employing anti-inflammatory and immunomodulatory agents such as 5-aminosalicylic acid, steroids, and thiopurines. However, 20-40\% of patients lose response or are nonresponders to these agents, which will lead to the use of biologics (i.e., anti-TNF agents) that may cause more severe adverse reactions, or surgical resection of the colon [3]. Given that nearly half of patients fail to achieve mucosal healing with available 
medications [11], we hypothesize that CTB-KDEL may provide a novel therapeutic option to achieve the major goal in UC treatment.

Although oral administration of CTB-KDEL has shown great promise in an acute DSS colitis mouse model, it is yet to be determined whether the treatment can reverse chronic colitis after it has been established. Acute DSS-induced colitis typically only incorporates features of innate immunity and acute epithelial injury [96]. In contrast, the chronic and progressive model of colitis induced by repeated exposure to DSS represents a complex interplay between innate immune responses and compromised epithelial barrier function that drives a chronic sustained inflammatory response [96, 149, 150].

Another potential issue for the therapeutic use of CTB-KDEL is that the parent molecule, CTB, is a strong mucosal immunogen [33-35]. In fact, CTB is used as a component of the World Health Organization (WHO) pre-qualified oral cholera vaccine Dukoral ${ }^{\circledR}$ to stimulate the production of both secretory immunoglobulin A (S-IgA), produced locally in the intestines, and serum immunoglobulin $\mathrm{G}(\mathrm{IgG})$ against CT [107, 151]. CTB's ability to induce a potent $\mathrm{S}-\operatorname{Ig} \mathrm{A}$ and $\operatorname{IgG}$ response upon mucosal administration is attributed to the various cell types within the GI tract that present GM1 ganglioside on their cell surface, such as macrophages, dendritic cells (DCs), B cells, T cells, neurons, and epithelial cells [36-39]. Previously we have determined that CTBKDEL's immunogenicity is equivalent to that of CTB in mice [89]. From a drug development standpoint, the induction of anti-drug antibodies (ADAs) is generally regarded as a risk because they could provoke clinical complications that arise from altered pharmacokinetics, pharmacodynamics, and/or immunotoxicity [152, 153]. However, it is yet to be determined whether immunogenicity is a relevant concern for 
orally administered biologics, as there are very few such agents that have undergone clinical investigation on this matter.

Here, using the repeated DSS exposure mouse colitis model, we investigated whether CTB-KDEL could alleviate chronic colitis and whether the therapeutic effects of CTB-KDEL are altered by the protein's immunogenicity. 


\subsection{Results}

4.2.1 CTB-KDEL's Therapeutic Effects in a Mouse Colitis Model Exposed to Repeated DSS Cycles.

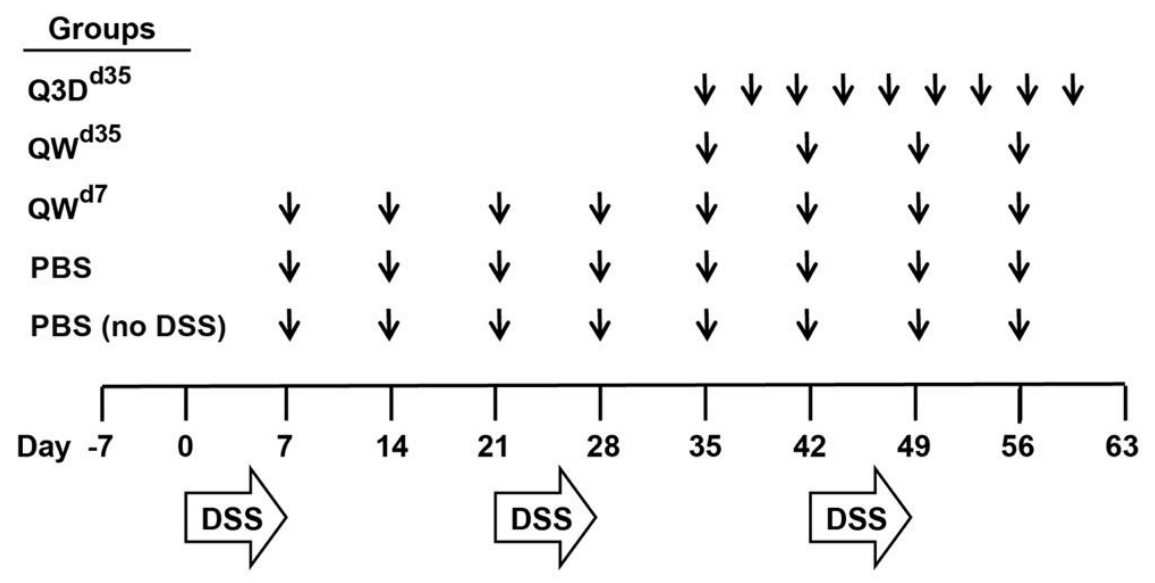

Figure 11 Dextran sodium sulfate (DSS) chronic colitis study design. Mice were exposed to three cycles of DSS that consisted of $2 \%$ DSS for seven days followed by 14 days of normal drinking water. Mice were treated orally with $3 \mu \mathrm{g}$ of cholera toxin $\mathrm{B}$ subunit (CTB)-KDEL or vehicle control; PBS, PBS + DSS, and QW ${ }^{\mathrm{d} 7}$ mice were dosed once a week starting at Day $7, \mathrm{QW}^{\mathrm{d} 35}$ mice were dosed once a week starting at Day 35, and $\mathrm{Q} 3 \mathrm{D}^{\mathrm{d} 35}$ mice were dosed every three days starting at Day 35. Disease activity index (DAI) scores were measured daily starting at Day 0. Following euthanasia on day 63, colon lengths were measured, serum was collected for fluorescein isothiocyanate (FITC)dextran analysis, and distal colon tissues were collected for cytokine quantification and histological examination.

To characterize the therapeutic effect of CTB-KDEL under chronic colitis conditions, we performed a chronic DSS colitis experiment in mice in which the animals were exposed to three cycles of seven-day DSS exposure followed by 14 days of normal 
drinking water over 63 days. In this chronic DSS-induced colitis model, mice develop chronic inflammation in the colon after the first cycle of DSS exposure [154]. Thus, mice were treated orally with $3 \mu \mathrm{g}$ of CTB-KDEL or vehicle control dosed once a week starting at Day $7\left(\mathrm{QW}^{\mathrm{d} 7}\right.$; before chronic colitis had developed), or dosed every three days or QW at Day $35\left(\mathrm{Q}^{3} \mathrm{D}^{\mathrm{d} 35}\right.$ and $\mathrm{QW}{ }^{\mathrm{d} 35}$, respectively) (Figure 11). As shown in Figure 12A, CTB-KDEL QW ${ }^{\mathrm{d} 7}$ administration significantly decreased disease activity index (DAI) scores immediately following the first dose and maintained significantly lower DAI values than the vehicle control-dosed group throughout the study despite exposure to two additional DSS cycles. Mice that were treated $\mathrm{QW}^{\mathrm{d} 35}$ or Q3D ${ }^{\mathrm{d} 35}$ (dosing started after chronic colitis was established) also showed immediate reduction in DAI scores following CTB-KDEL administration. On Day 63 mice were administered fluorescein isothiocyanate (FITC)-dextran by gavage $4 \mathrm{~h}$ prior to euthanasia to test the intestinal permeability of each group. Chronic DSS-induced colitis is known to induce barrier dysfunction followed by increased intestinal permeability that is correlated with worsening of the disease [149]. Compared to mice treated with vehicle control (PBS), those that received $\mathrm{QW}{ }^{\mathrm{d} 7}$ and $\mathrm{Q} 3 \mathrm{D}^{\mathrm{d} 35}$ CTB-KDEL treatment showed significantly lower plasma FITC-dextran levels, indicating that CTB-KDEL treatment improved intestinal barrier function (Figure 12B). QW ${ }^{\mathrm{d} 35}$ treatment also appeared to have improved the barrier function; however, the decrease in FITC-dextran plasma levels was not statistically significant. Likewise, with $\mathrm{QW}^{\mathrm{d} 7}$ and $\mathrm{Q} 3 \mathrm{D}^{\mathrm{d} 35}$, but not with $\mathrm{QW}^{\mathrm{d} 35}$ dosing, CTB-KDEL significantly prevented colon shrinkage induced by DSS insult (Figure 12C). 

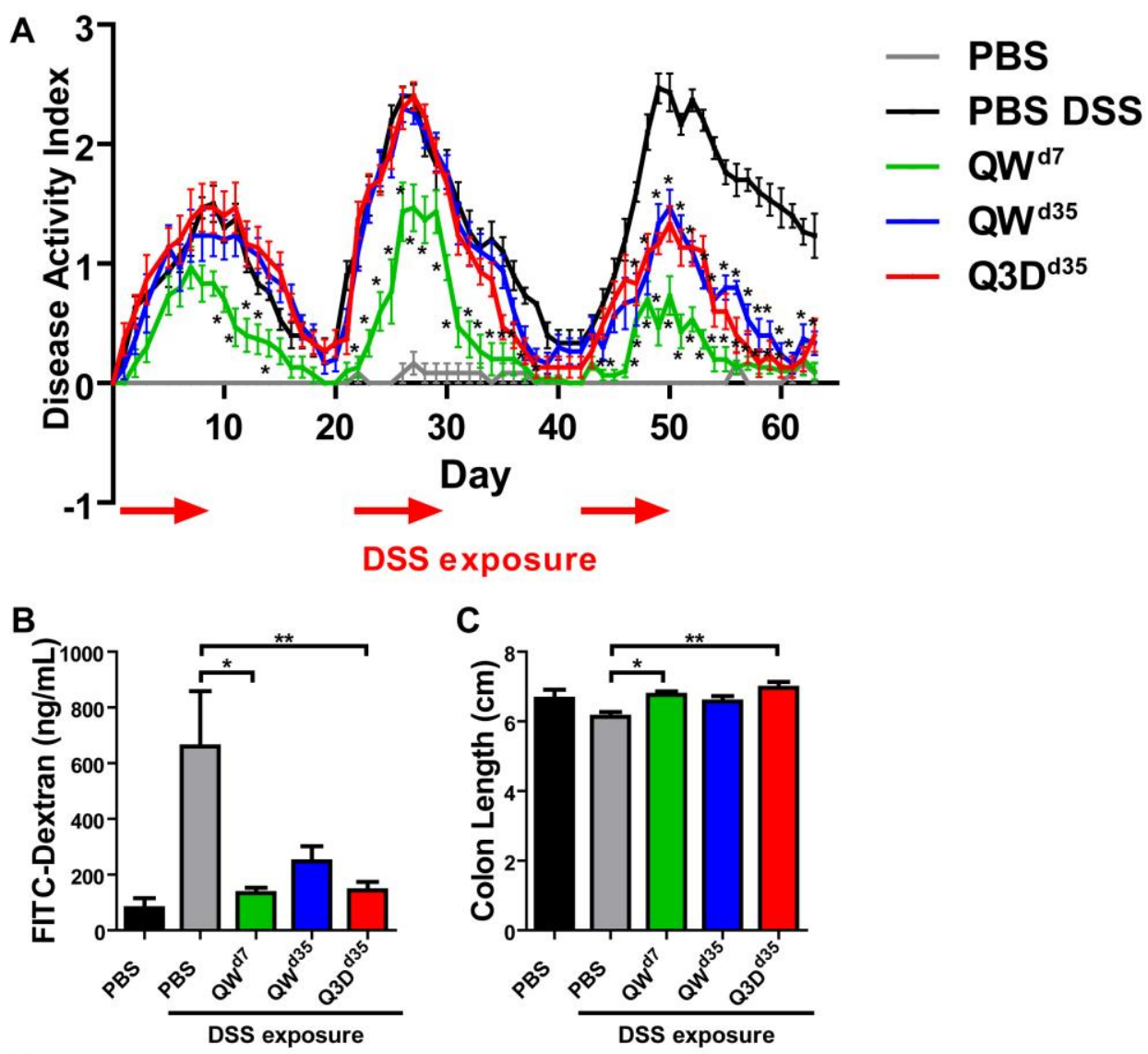

C
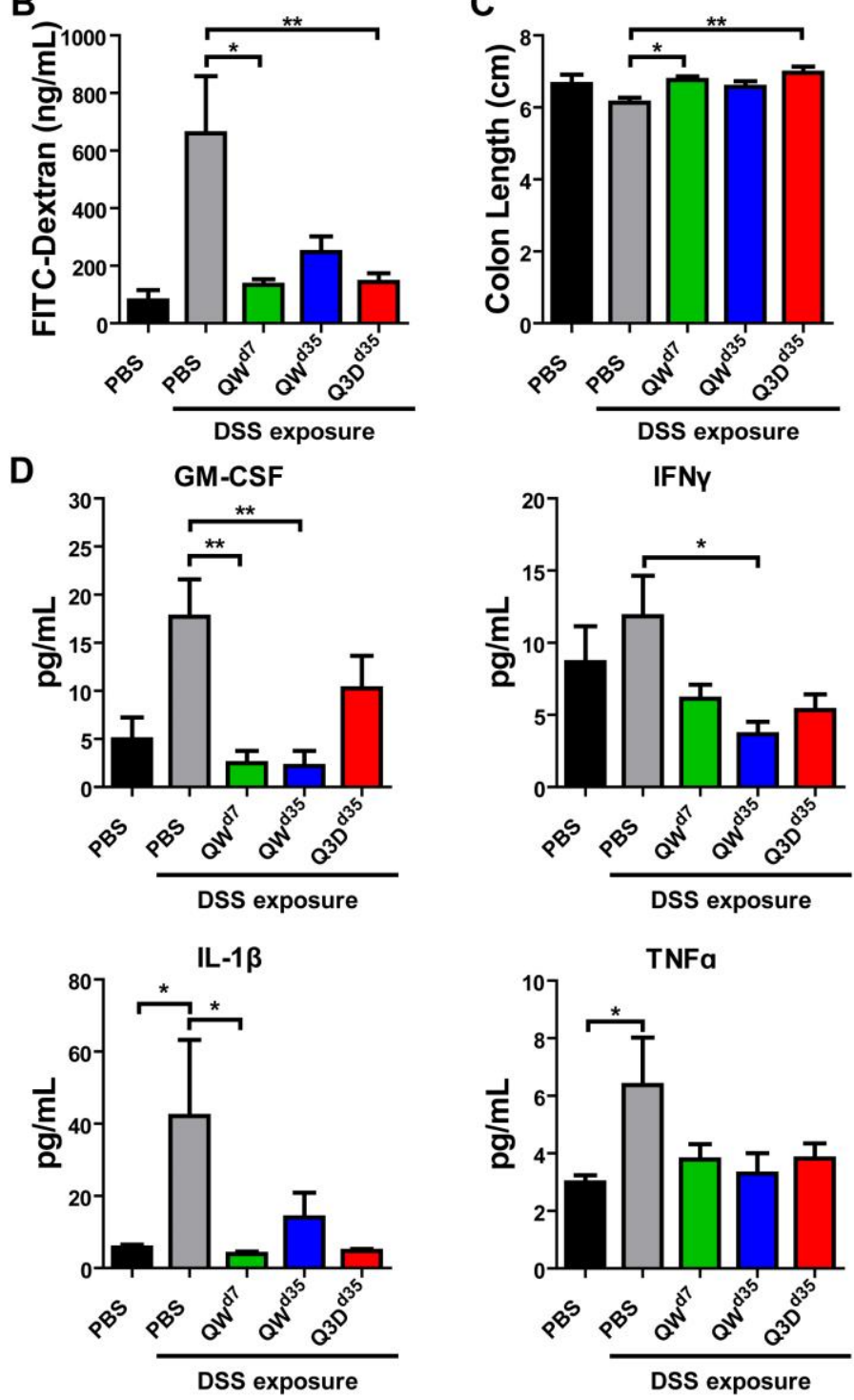


\section{Figure 12. CTB-KDEL mitigates DSS-induced injury and inflammation in a DSS}

chronic colitis study. C57BL/6 mice $(n=10)$ were exposed to three cycles of seven-day 2\% DSS and 14-day normal drinking water over 63 days and treated with $3 \mu \mathrm{g}$ CTBKDEL or PBS dosed orally every week starting at Day $7\left(\mathrm{QW}^{\mathrm{D} 7}\right)$ or starting at Day 35 dosed once a week $\left(\mathrm{QW}^{\mathrm{D} 35}\right)$ or every three days $\left(\mathrm{Q}^{\mathrm{D}} \mathrm{D}^{\mathrm{D} 35}\right)$. (A) The time course of disease activity index (DAI) values (mean \pm S.E.M.) in each group measured from Days 0-63. *p $<0.05$; two-way repeated measures ANOVA with Bonferroni's multiple comparison tests. (B) FITC-dextran concentrations found in mice serum $4 \mathrm{~h}$ after administration on Day 63. (C) Colon length measured after Day 63 sacrifice. (D) Proinflammatory cytokines in distal colon tissues were measured by Luminex after Day 63 sacrifice. (B, C, D) $* p<0.05, * * p<0.01$; one-way repeated measures ANOVA with Bonferroni's multiple comparison tests.

To dissect the therapeutic effects of CTB-KDEL, we measured pro-inflammatory cytokine levels in the distal colon tissue lysates at the time of euthanasia (Day 63). Consistent with other reports of chronic colitis $[155,156]$, two key markers of chronic colitis IL-1 $\beta$ and TNF $\alpha$ were significantly elevated in PBS-treated mice when compared to healthy animals (Figure 12D). In contrast, none of the CTB-KDEL treatment groups demonstrated an elevation of the pro-inflammatory cytokines tested; IL-1 $\beta$, TNF $\alpha$, GMCSF, and IFN $\gamma$, suggesting an overall suppression of inflammation by CTB-KDEL treatment. In particular, $\mathrm{QW}{ }^{\mathrm{d} 7}$ treatment showed significantly lower GM-CSF and IL-1 $\beta$ levels compared to mice treated with PBS + DSS, while QW ${ }^{\mathrm{d} 35}$ mice had significantly reduced GM-CSF and IFN $\gamma$ cytokine levels compared to mice treated with PBS + DSS. 
Although Q3 $\mathrm{D}^{\mathrm{d} 35}$ dosing did seem to reduce pro-inflammatory cytokine levels, these reductions were not statistically significant.

To further corroborate the above results, we performed a histopathological examination and evaluated the presence of active chronic colitis markers, particularly crypt structural alterations and expansion of the lamina propria, in hematoxylin and eosin (H\&E)-stained distal colon tissues. By these metrics, CTB-KDEL-treated mice showed increased recovery from chronic DSS-induced colitis compared to vehicle control. QW ${ }^{\mathrm{d} 7}$ and $\mathrm{Q} 3 \mathrm{D}^{\mathrm{d} 35}$ dosing demonstrated similar but noteworthy recovery, exhibiting fewer instances of crypt branching, crypt distortion, and expansion of the lamina propria than $\mathrm{QW}^{\mathrm{d} 35}$ dosing (Figure 13A). It was indeterminable whether $\mathrm{QW}^{\mathrm{d} 7}$ or $\mathrm{Q} 3 \mathrm{D}^{\mathrm{d} 35}$ dosing had the greatest impact on recovery from chronic colitis. $\mathrm{QW}^{\mathrm{d} 35}$ dosing, however, still showed signs of recovery compared to vehicle control, as the latter displayed the most severe expansion of the lamina propria and crypt alterations (Figure 13A). These histopathological findings were quantified by a crypt score rating of the H\&E-stained distal colon tissues. As shown in Figure 13B, all CTB-KDEL-treated groups had a significantly reduced crypt score, which was characterized by a reduction in the shortening or loss of basal crypts and epithelial surface retention in all CTB-KDELtreated mice, in contrast to PBS treatment (Figure 13B). 
A
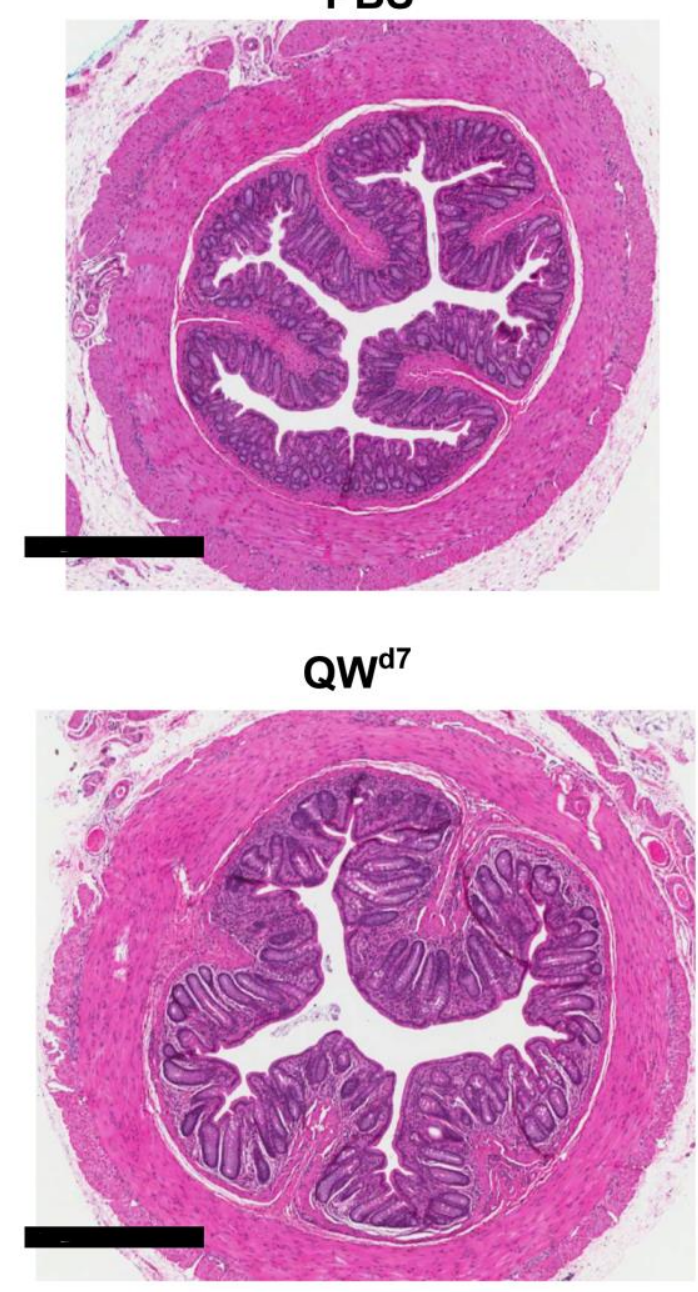

Q3D ${ }^{\mathrm{d} 35}$

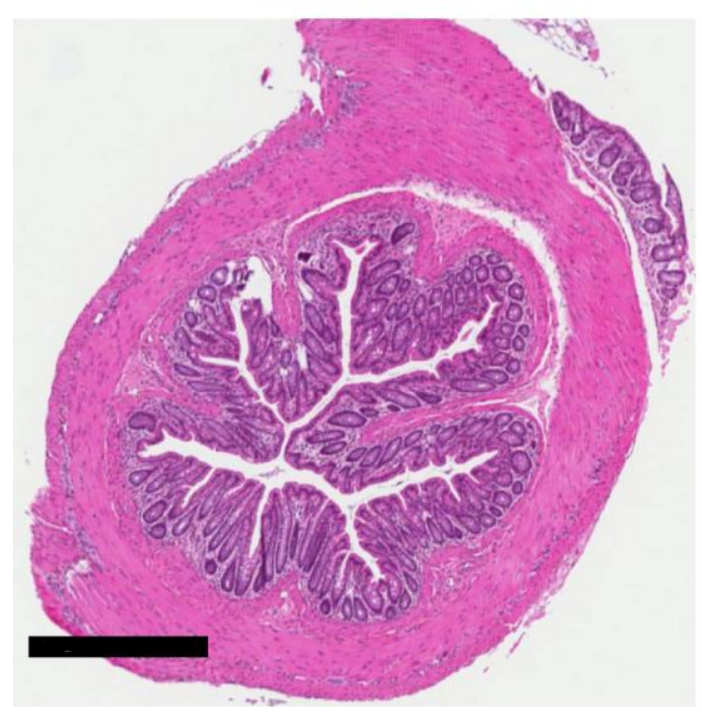

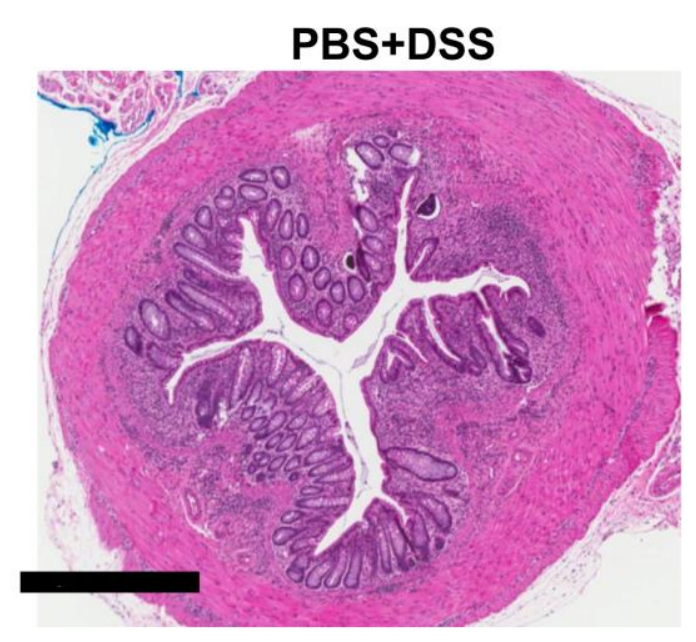
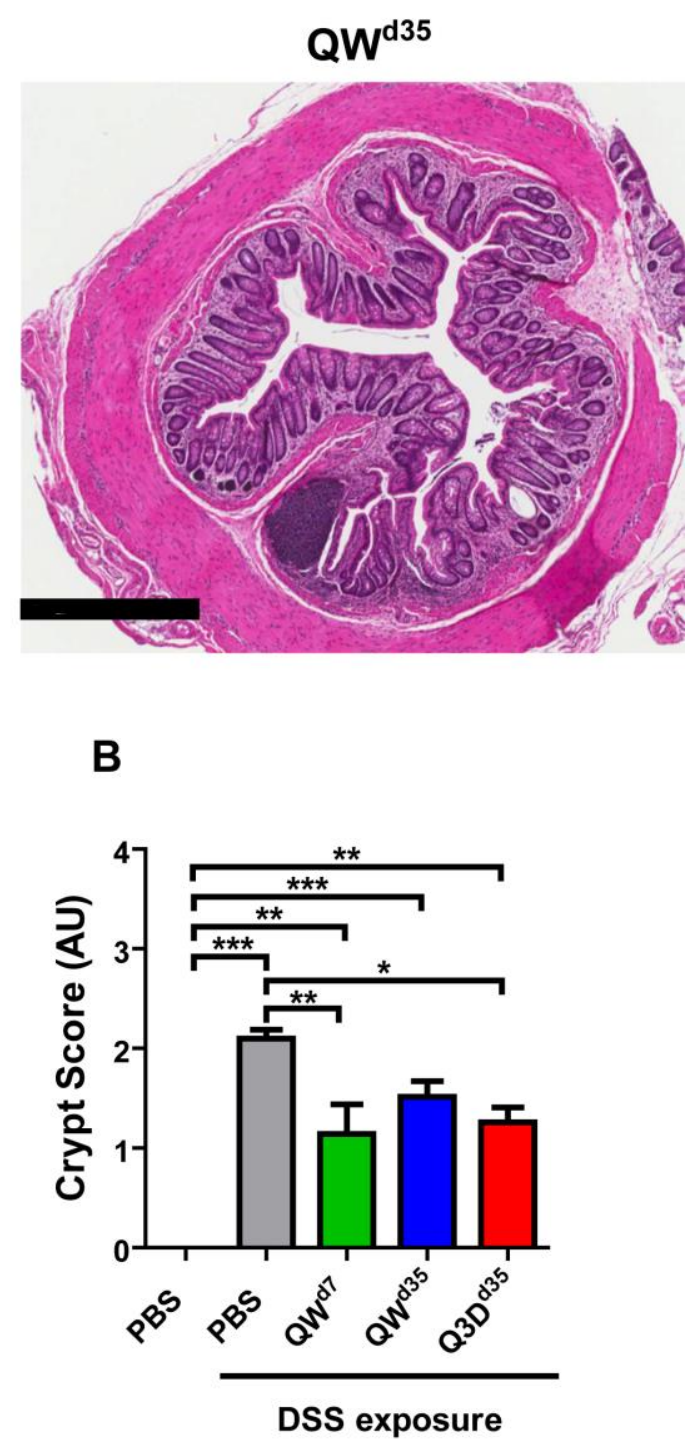


\section{Figure 13. Histological findings from the DSS chronic colitis model. CTB-KDEL}

treatment protected mice from inflammation and loss of colonic epithelial surface and crypts. (A) Representative $4 \times$ photomicrographs of hematoxylin and eosin (H\&E)-stained distal colon tissues from mice treated with $3 \mu \mathrm{g}$ CTB-KDEL or PBS dosed orally every week starting at Day $7\left(\mathrm{QW}^{\mathrm{D} 7}\right)$ or starting at Day 35 dosed once a week $\left(\mathrm{QW}^{\mathrm{D} 35}\right)$ or every three days $\left(\mathrm{Q}^{\mathrm{D}} \mathrm{D}^{\mathrm{D} 35}\right)$ starting at Day 35. Scale bars $=600 \mu \mathrm{M}$. (B) Colon crypt scoring from paraffin-embedded tissue sections were scored after H\&E staining. Scoring was based on a $0-4$ scale. ${ }^{*} \mathrm{p}<0.05,{ }^{* *} \mathrm{p}<0.01, * * * \mathrm{p}<0.001$; one-way repeated measures ANOVA with Bonferroni's multiple comparison tests.

4.2.2 The Impact of CTB-KDEL's Immunogenicity on its Therapeutic Effect in Acute DSS Colitis Models.

Previously, we have shown that oral administration of 3,10 , or $30 \mu \mathrm{g}$ CTB-KDEL twice at a two-week interval induced anti-toxin serum IgG and fecal IgA antibodies with high titers [89]. Notwithstanding, the results in the chronic DSS colitis study shown in Figures 2 and 3 demonstrate that repeated dosing with $3 \mu \mathrm{g}$ of CTB-KDEL (eight doses total) did not lose efficacy over the course of the study, raising an interesting question about the consequences of an immune response to the protein. Thus, to address this immunogenicity question, specifically, the induction of ADAs, we used a mouse model of acute DSS colitis in which mice were orally administered with $30 \mu \mathrm{g}$ of CTB-KDEL twice prior to $3 \%$ DSS exposure to induce an immunogenic response to the protein (the aforementioned vaccine dosing regimen [89]). At the end of DSS exposure, mice were orally administered $3 \mu \mathrm{g}$ of CTB-KDEL; this single therapeutic dose has proven to be 
efficacious in C57BLl/6J mice [89, 147]. The dosing regimens and groups are depicted in Figure 14.

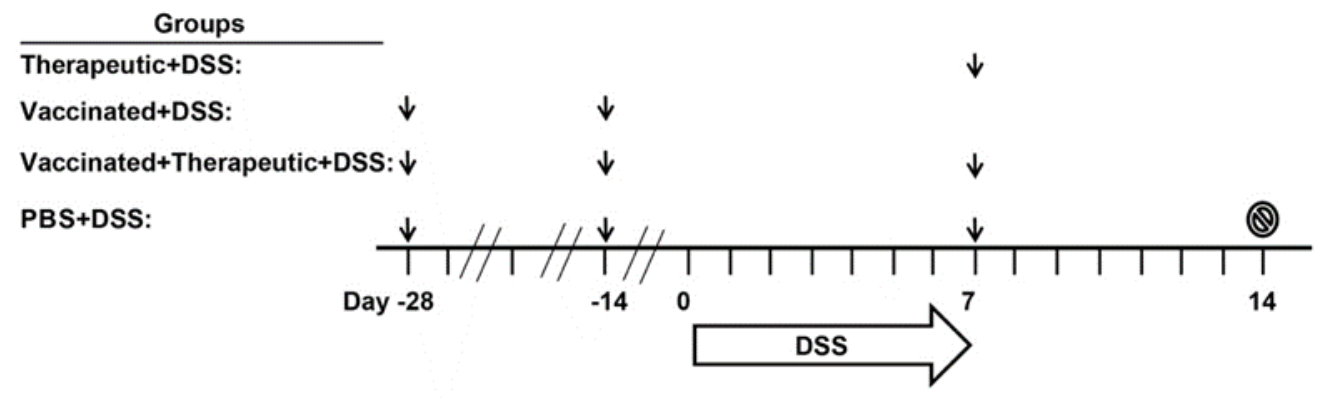

Figure 14. Immunogenicity and acute DSS colitis study design. Four groups of mice were studied; PBS (healthy vehicle control), PBS + DSS (vehicle control in diseased mice), vaccinated + DSS, therapeutic + DSS, and vaccinated + therapeutic+DSS. All mice except for Therapeutic + DSS mice were vaccinated with $30 \mu \mathrm{g}$ CTB-KDEL or PBS 28 and 14 days (Days -28 and -14 ) prior to DSS administration. On Day 0, mice were given $3 \%$ DSS in drinking water for seven consecutive days. On the seventh day of DSS exposure, DSS was removed from the drinking water and then PBS, PBS + DSS, therapeutic + DSS, and vaccinated + therapeutic + DSS mice were administered with 3 $\mu \mathrm{g}$ CTB-KDEL or a vehicle control (PBS) in a volume of $100 \mu \mathrm{L}$ after sodium bicarbonate (200 $\mu \mathrm{L}$ of $30 \mathrm{mg} / \mathrm{mL}$ solution) administration. Seven days later, on Day 14, mice were euthanized (stop sign) and colon lengths and a disease activity index scores were measured. Body weights were measured daily and feces were collected on Days 7 and 14 from each mouse. Black arrows represent the timings of oral administration of CTB-KDEL or PBS. 
As shown in Figure 15A, after DSS exposure was halted all three CTB-KDEL dosing regimens showed a significantly more rapid recovery from DSS-induced weight loss when compared to PBS-treated mice. Vaccinated + therapeutic + DSS mice showed a significant improvement one day after therapeutic dosing (Day 8), while therapeutic + DSS and vaccinated + DSS mice showed a significant improvement in weight recovery starting at Day 10 and continued throughout the study. These results were confirmed by colon length and DAI scores that were examined upon euthanasia at Day 14. The colon lengths of both groups therapeutically treated with CTB-KDEL on Day 7 were significantly longer than those of the PBS + DSS group (Figure 15B). Additionally, all CTB-KDEL treated mice showed similar DAI scores, which were significantly lower than those of PBS+DSS mice (Figure 15C). To determine the presence of ADAs in the GI tract of these mice, we performed an ELISA detecting CTB-binding IgAs in feces collected before (Day 7) and after (Day 14) the therapeutic dose of CTB-KDEL was administered. Both vaccinated + DSS and vaccinated + therapeutic + DSS mice had similar and high levels of anti-CTB-KDEL IgA antibodies in the intestine on both days, 
indicating that ADAs were present when CTB-KDEL was administered for the treatment of colitis (Figure 15D).
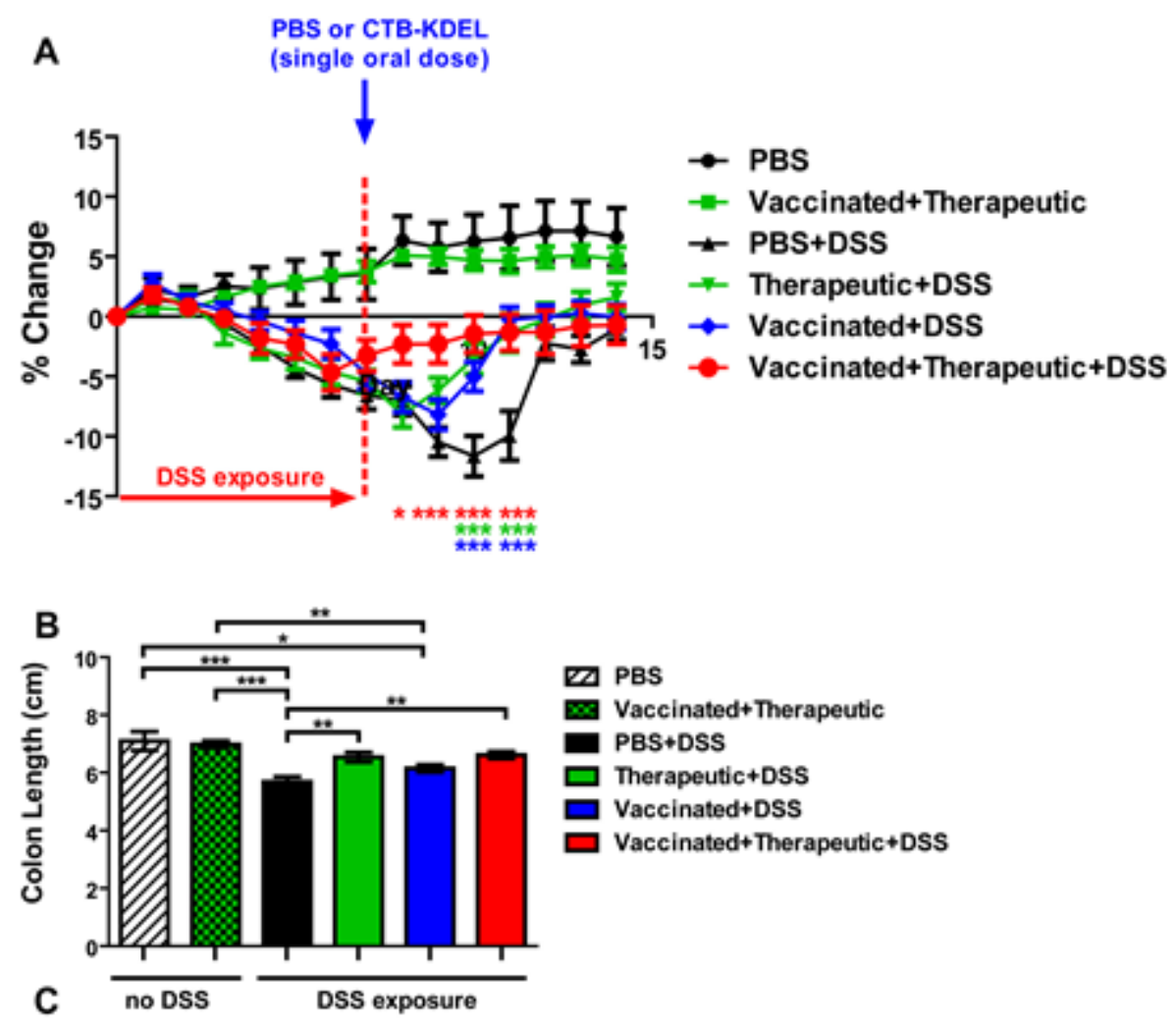

ED PBS

Vaccinated + Therapeutic

aBs+DSS

$\square$ Therapeutic+DSS

$\square$ Vaccinated+DSS

$\square$ Vaccinated + Therapeutic + DSS

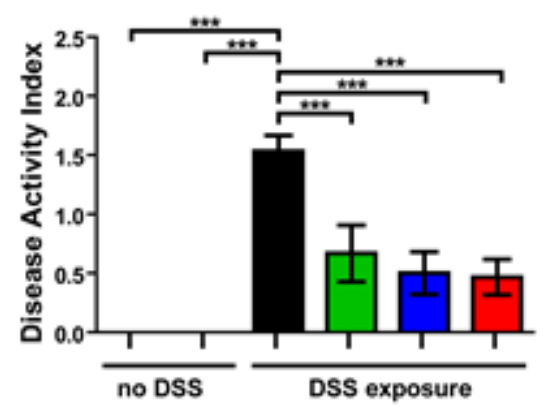

20 PBS

Eaccinated + Therapeutic

PBS+DSS

$\square$ Therapeutic+DSS

Vaccinated+DSS

$\square$ Vaccinated+Therapeutic+DSS

D

Anti-CTB-KDEL IgA Titers: Day 7

Anti-CTB-KDEL IgA Titers: Day 14
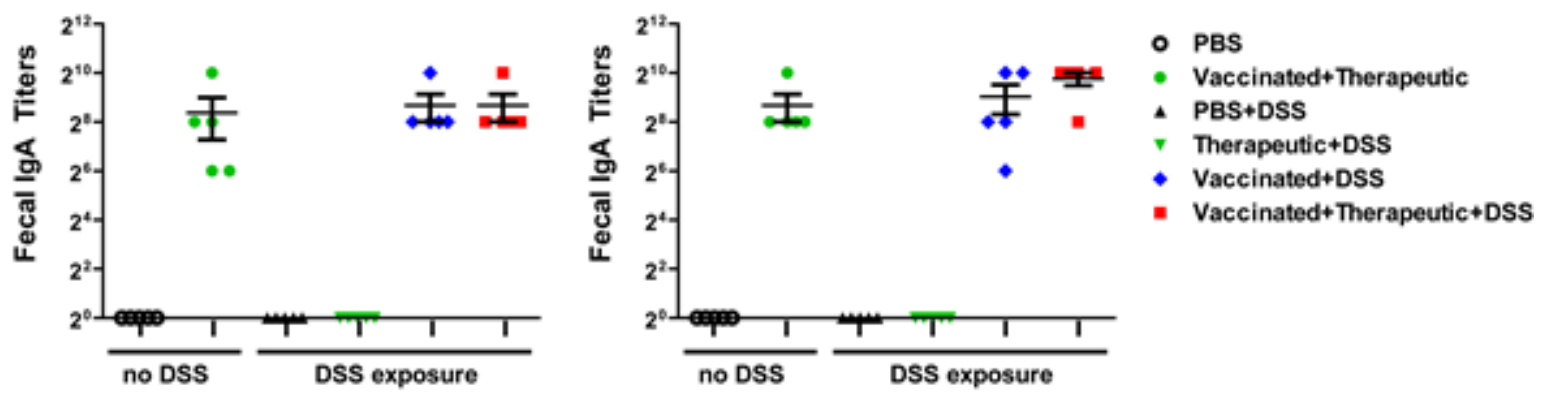
Figure 15. The impact of a pre-existing immune response to CTB-KDEL on its therapeutic efficacy against DSS acute colitis in C57BL/6 mice. C57BL/6 mice were pre-dosed twice orally with $30 \mu \mathrm{g}$ CTB-KDEL or PBS on Day -28 and Day -14 and then exposed to DSS on Days 0-7. A therapeutic dose of CTB-KDEL (3 $\mu \mathrm{g})$ was dosed orally on Day 7. (A) Percent body weight change was monitored daily. Two-way ANOVA with Bonferroni's multiple comparison tests were used to compare groups. ${ }^{*} p<0.05,{ }^{*} p<$ $0.01, * * * \mathrm{p}<0.001$, compared to PBS + DSS group. (B) Colon length measured at sacrifice on Day 14. (C) Disease activity index (DAI) at sacrifice on Day 14. *p $<0.05$, $* * \mathrm{p}<0.01, * * * \mathrm{p}<0.001$. One-way ANOVA with Bonferroni's multiple comparison tests. (D) Fecal anti-CTB-KDEL IgA titers were determined by ELISA on Days 7 (left) and 14 (right).

To further reveal the impacts of ADAs, we performed the same acute DSS colitis experiment in $\mathrm{C} 3 \mathrm{H} / \mathrm{HeJ}$ mice which, unlike the $\mathrm{C} 57 \mathrm{BL} / 6$ strain, are poor responders to CTB immunization due to the lack of an MHC haplotype reactive to CTB epitopes [157, 158]. The experiment was performed exactly as described above (Figure 14) and the same dosing groups were implemented. Similar to the results obtained in C57BL/6 mice, all three CTB-KDEL dosing regimens proved to be efficacious against DSS colitis in contrast to PBS-treated mice (Figure 16). A significant prevention of body weight loss was noted in vaccinated + DSS as early as Day 6, while vaccinated + therapeutic + DSS and therapeutic + DSS mice demonstrated a significant recovery from body weight loss starting on Day 8 (Figure 16A). Furthermore, all CTB-KDEL-treated mice showed significantly reduced colon shrinkage and lower DAI scores compared to PBS + DSS 
mice and were comparable between all CTB-KDEL treatment groups (Figure 16B-C).

Unlike C57BL/6 mice, however, $\mathrm{CH} 3 / \mathrm{HeJ}$ mice had little to no anti-CTB IgA response to 
the vaccination with CTB-KDEL (Figure 16D). Thus, CTB-KDEL's mucosal therapeutic effects in this model are unlikely to be dependent on $\operatorname{Ig} \mathrm{A}$ induction in the intestine.

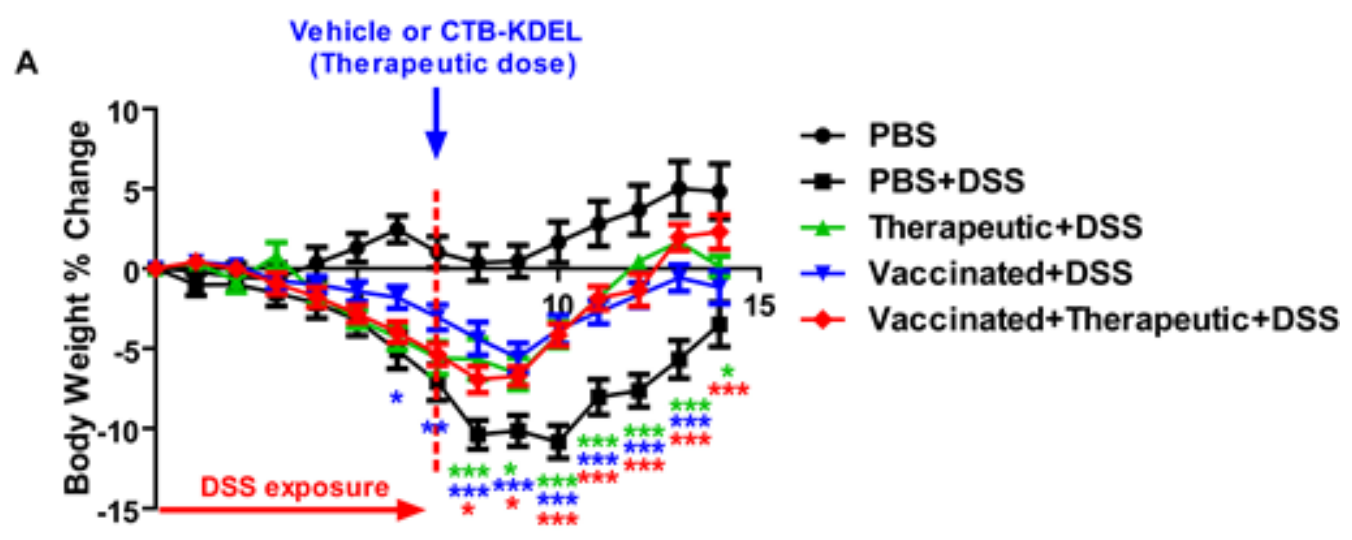

B

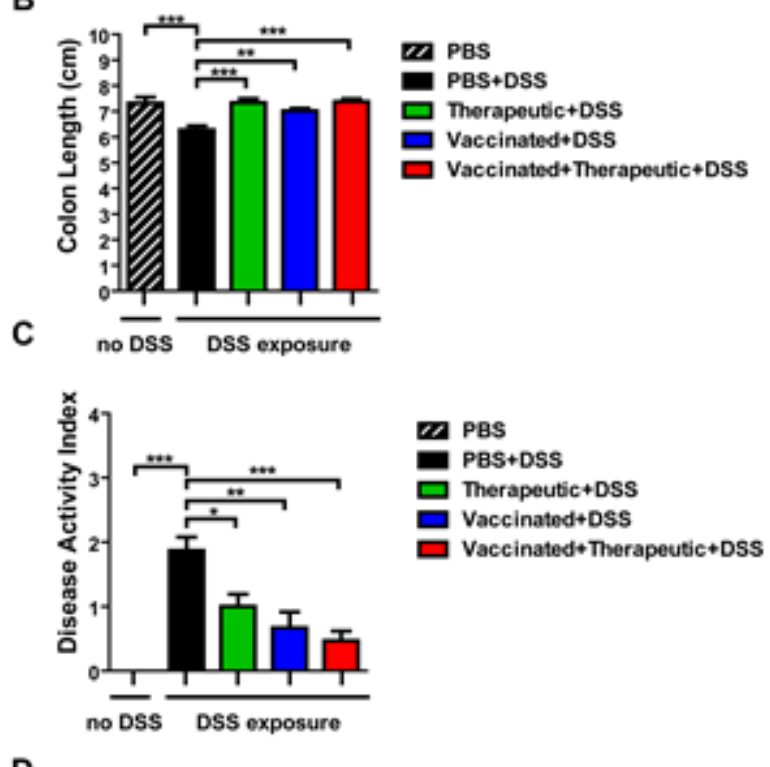

D

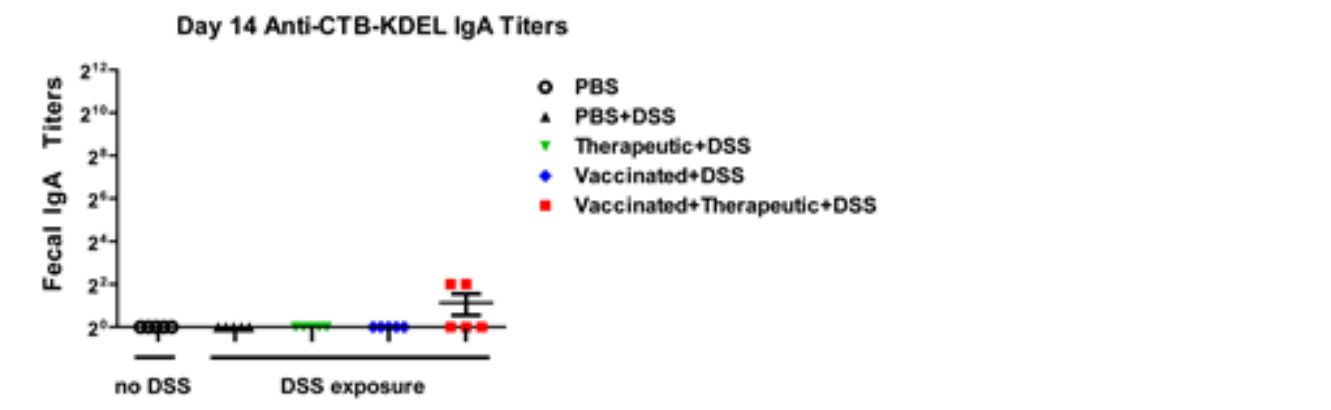

Figure 16. The impact of CTB-KDEL predosing on its therapeutic efficacy against

DSS acute colitis in $\mathbf{C 3 H} / \mathrm{HeJ}$ mice. $\mathrm{C} 3 \mathrm{H} / \mathrm{HeJ}$ mice were pre-dosed twice orally with 30 
$\mu \mathrm{g}$ CTB-KDEL or PBS on Day -28 and Day -14 and then exposed to DSS on Days 0-7. A therapeutic dose of CTB-KDEL ( $3 \mu$ g) was dosed orally on Day 7. (A) Percent body weight change was monitored daily. Two-way ANOVA with Bonferroni’s multiple comparison tests were used to compare groups. ${ }^{*} \mathrm{p}<0.05$, $* * \mathrm{p}<0.01$, *** $\mathrm{p}<0.001$, compared to PBS + DSS group. (B) Disease activity index (DAI) at sacrifice on Day 14. (C) Colon length measured at sacrifice on Day 14. ${ }^{*} \mathrm{p}<0.05$, $* * \mathrm{p}<0.01,{ }^{* * *} \mathrm{p}<0.001$. One-way ANOVA with Bonferroni's multiple comparison tests. (D) Fecal anti-CTBKDEL IgA titers were determined by ELISA on Day 14.

Finally, to test if the adaptive immune system is dispensable in CTB-KDEL's therapeutic benefit in the mitigation of DSS colitis we tested the effects of CTB-KDEL in Ragl ${ }^{-/}$mice, which are deficient in mature B or $\mathrm{T}$ cells[159], with the same experimental design as performed in $\mathrm{C} 57 \mathrm{BL} / 6$ and $\mathrm{C} 3 \mathrm{H} / \mathrm{HeJ}$ mice (Figures 14, 15, and 16). In contrast to $\mathrm{C} 57 / \mathrm{BL} 6$ and $\mathrm{C} 3 \mathrm{H} / \mathrm{HeJ}$ mice, $\mathrm{Rag} 1^{-/-}$mice did not show a robust effect in body weight by CTB-KDEL treatment (Figure 17). Only Vaccinated+Therapeutic+DSS group showed a significant recovery on Day 10 (Figure 17A). Upon sacrifice on Day 14, there was no significant difference between the colon lengths of CTB-KDEL treated mice and vehicle control group. However, DAI scores of therapeutic+DSS and vaccinated+therapeutic+DSS mice were significantly lower than those of PBS+DSS treated mice (Figure 17B). When DAI scores were decomposed into individual variables, a significant decrease in stool consistency scores was noted in the groups therapeutically treated with CTB-KDEL (vaccinated+therapeutic+DSS and Therapeutic+DSS), an indicator of decreased intestinal permeability caused by DSS-injury (Figure 17D). There 
was no significant difference in blood in stool between CTB-KDEL treated and PBS+DSS treated mice. These results confirm CTB-KDEL's therapeutic effect is not dependent on a host IgA response; however, further research is needed to determine why CTB-KDEL's effect is not as robust in Rag1-/- mice as C57BL/6 and C3H/HeJ mice.
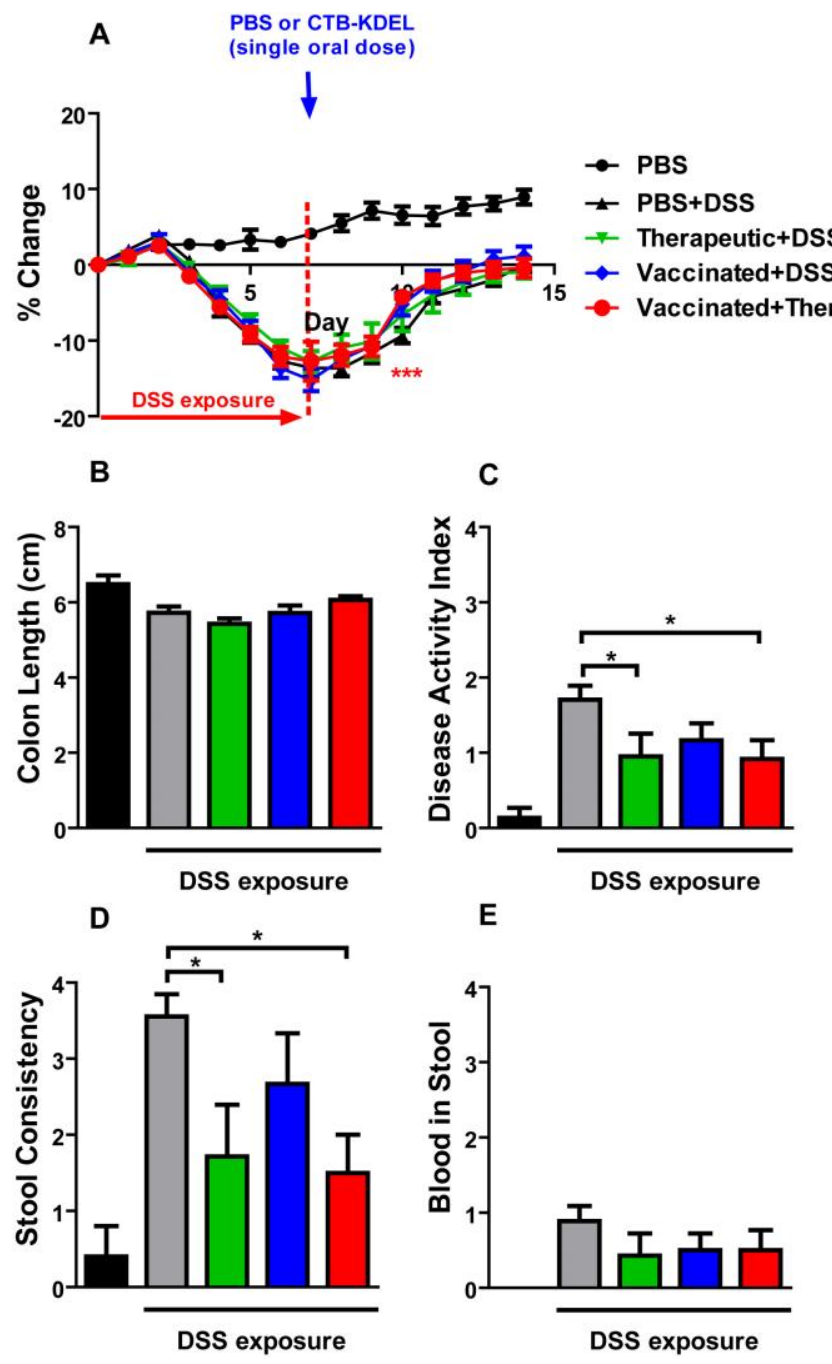

F Day 14 Anti-CTB-KDEL IgA Titers

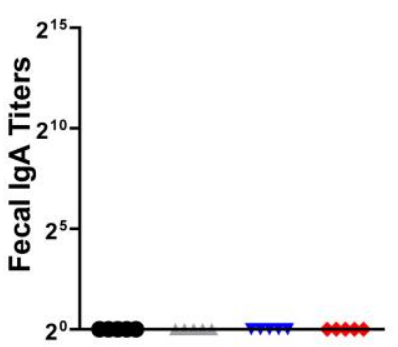

PBS

$\square$ Therapeutic+DSS

$\square$ Vaccinated+DSS

$\square$ Vaccinated+Therapeutic+DSS

Figure 17. Analysis of CTB-KDEL's therapeutic efficacy against DSS acute colitis in immunodeficient mice. Rag1-/- mice were pre-dosed twice orally with $30 \mu \mathrm{g}$ CTB-

KDEL or PBS on Day -28 and Day -14 and then exposed to DSS on Days $0-7$. A 
therapeutic dose of CTB-KDEL ( $3 \mu \mathrm{g}$ ) was dosed orally on Day 7. (A) Percent body weight change was monitored daily. Two-way ANOVA with Bonferroni's multiple comparison tests were used to compare groups. ${ }^{*} \mathrm{P}<0.05$, $* * \mathrm{P}<0.01$, *** $\mathrm{P}<0.001$, compared to PBS + DSS group. (B) Disease activity index (DAI) at sacrifice on Day 14 . (C) Colon length measured at sacrifice on Day 14. (D) Stool Consistency measured at sacrifice on Day 14. (E) Blood in stool measured at sacrifice on Day 14. *P $<0.05$, **P $<0.01, * * * \mathrm{P}<0.001$. One-way ANOVA with Bonferroni's multiple comparison tests. (F) Fecal anti-CTB-KDEL IgA titers were determined by ELISA on 14. 


\subsection{Discussion}

We have previously demonstrated CTB-KDEL's mucosal healing effects in an acute DSS colitis model in C57BL/6 mice and in colonic explants isolated from IBD patients, as well as the prevention of tumor development in an azoxymethane $(\mathrm{AOM}) / \mathrm{DSS}$ mouse model of colitis-associated cancer. However, its development as a biotherapeutic will need to address unique issues associated with its use. Here we aimed to uncover two key questions surrounding CTB-KDEL's ability to treat chronic symptoms of UC. Specifically, we addressed whether CTB-KDEL could resolve colitis after the formation of chronic inflammatory conditions in a more clinically relevant model based on repeated DSS exposure. Additionally, we determined the impact of immunogenicity on CTB-KDEL's therapeutic efficacy, as the protein is a potent inducer of mucosal and systemic antibodies.

Acute DSS-induced colitis typically only incorporates features of innate immunity and acute epithelial injury [160]. In contrast, the chronic and progressive model of DSS colitis presented in this manuscript represents a complex interplay between innate immune responses and compromised epithelial barrier function that drives a chronic sustained inflammatory response [96]. The inflammatory response is driven mainly through the paracellular route created by DSS exposure that is exacerbated by proinflammatory cytokines, such as TNF $\alpha$ and INF $\gamma[161,162]$. After repeated DSS cycles, this proinflammatory response leads to a positive feedback loop of increased intestinal permeability, initially driven by DSS, and increased production of inflammatory cytokines in response to bacteria that cross the intestinal barrier and 
inflammatory cytokines that also cause direct damage to the intestinal epithelium $[160$, 163, 164]. Apoptosis of intestinal epithelial cells and subsequent damage to intestinal mucosa magnifies an increase in intestinal permeability, further adding to the positive feedback loop between increased intestinal permeability, enhanced production of inflammatory cytokines, and damaged intestinal epithelium. For these reasons, we initiated oral CTB-KDEL treatment at two different timepoints during the nine-week chronic DSS colitis experiment. One group received the first CTB-KDEL dose at the end of the first DSS cycle (i.e., Day 7) when the DSS--induced injury was in the acute phase, whereas two other groups initiated CTB-KDEL treatment after the second cycle of DSS (i.e., Day 35) when more severe colitis had started to develop. The chronic inflammation and histopathogical findings seen in the vehicle control group are similar to the features of those seen in human UC [10,35], demonstrating the relevance of this model for the purpose of the present study. The data revealed that $\mathrm{QW}^{\mathrm{d} 7}$ and $\mathrm{Q} 3 \mathrm{D}^{\mathrm{d} 35}$ dosing of $3 \mu \mathrm{g}$ CTB-KDEL were more efficacious than $\mathrm{QW}^{\mathrm{d} 35}$ dosing (Figure 12). Although least effective among the three CTB-KDEL treatment groups, the $\mathrm{QW}^{\mathrm{d} 35}$ dosing group still showed efficacy, as evident from significantly low DAI values compared to the untreated group throughout the last DSS exposure cycle. Meanwhile, dosing every two weeks was not as effective as QW dosing regardless of the timing of initial treatment (data not shown). The therapeutic efficacy of CTB-KDEL against chronic colitis was corroborated by histological evidence for significant remission and healing in mice treated with the protein. In addition, it was also noted that there was minimal neutrophil infiltration in tissues of CTB-KDEL-treated mice in contrast to vehicle control (Figure 13). The chronic inflammation and histopathological findings seen in our vehicle control group are similar to the features of those seen in human UC [96, 165]. Furthermore, these results 
add to the clinical relevance of the present study because histologic remission and healing is an area of increased research focus and holds the promise of being an important marker of treatment efficacy in UC $[166,167]$. Of note, all CTB-KDEL-dosed groups showed reduction in intestinal permeability (serum FITC-dextran levels), downregulation of proinflammatory cytokine levels and decreased histological damage in the colon, all three of the main hallmarks of IBD $[155,160,168]$.

The significance of the above results is twofold. First, the results indicate that the animals continued to respond to the medication over time. Conversely, CTB-KDEL did not lose its efficacy with repeated dosing (eight doses total for $\mathrm{QW}^{\mathrm{d} 7}$, four doses for $\mathrm{QW}^{\mathrm{d} 35}$, and nine doses for $\mathrm{Q}^{\mathrm{D}} \mathrm{D}^{\mathrm{d} 35}$ ). Second, as the repeated DSS model usually causes chronic inflammation in the colon after the first cycle of DSS exposure [154], the positive results in the delayed dosing groups indicate that CTB-KDEL is effective against chronic colitis. Collectively, these data provide a basis for both QW and Q3 ${ }^{\mathrm{d} 35}$ dosing to be used in the treatment of chronic colitis; QW dosing may be sufficient as a maintenance dose to prevent relapse of disease, while Q3D dosing may be better suited for treatment of active disease.

A key pharmacological and toxicological question for biotherapeutic development is immunogenicity; specifically, ADA induction. A major disadvantage of therapeutic proteins is that almost all induce an antibody response [169]. ADAs constitute a theoretical risk because they may lead to a loss of efficacy, altered pharmacokinetics, and potentially cause immunotoxicity $[152,153,170,171]$. As CTB-KDEL is a derivative of the cholera vaccine antigen CTB, it is of critical importance to assess the consequences of an immune response to CTB-KDEL on its pharmacological and/or toxicological 
parameters for its development as a drug for chronic diseases like UC, which would likely need repeated and long-term medication.

Typically, nonclinical models of immunogenicity are not always predictive of immune responses in humans [171]. However, in most cases, the studies are performed to identify the possibility of ADA induction and determine the capacity of ADAs, if elicited, to affect the pharmacokinetics and pharmacodynamics of the drug. It is already known that CTB is immunogenic in humans as well as in rodents [107]. Hence, we used multiple strains of mice to reveal the dynamics of CTB-KDEL's immunogenicity on its therapeutic effects in colitis. To that extent, the study using C57BL/6 mice represented a scenario of high $\mathrm{ADA}$ induction, while $\mathrm{C} 3 \mathrm{H} / \mathrm{HeJ}$ mice provided a scenario of minimal ADA induction. Both scenarios were deemed important for the implications of CTBKDELs intestinal therapeutic effect. Nevertheless, CTB-KDEL was efficacious in both species of mice indicating the consequences of immunogenicity on its ability to treat UC are negligible.

In the acute DSS colitis experiment in which C57BL/6 mice were pre-dosed twice with CTB-KDEL (i.e., "vaccinated"), we indeed observed significant levels of anti-CTBKDEL IgA antibodies in the intestine before DSS exposure and throughout the rest of the study (Figure 15D). Although we did not directly analyze the capacity of the ADAs to neutralize CTB-KDEL's effect, the therapeutic efficacy observed in Vaccinated+Therapeutic+DSS group was not inferior to that of Therapeutic+DSS group, suggesting that intestinal ADAs arising from repeated CTB-KDEL administrations have limited impacts, if any, on the protein's mucosal healing efficacy. However, 
interpretation of the results from this experiment is complicated by the fact that the CTBKDEL vaccination also showed marked prophylactic effects against DSS colitis. Our preliminary study show that CTB-KDEL is only detectable in the colon up to 48 hours after a single oral administration in CTB-KDEL naïve mice (data not shown). Thus, the prophylactic effect of CTB-KDEL seen in Figure 15 could be due to the ADAs extending the bioavailability of the protein in the colon. Alternatively, the induction of ADAs itself might have played a beneficial role in mitigating inflammation because IgA is known to help maintain mucosal homeostasis and/or mediate anti-inflammatory functions $[73,74,172-174]$. In fact, this was previously demonstrated in CTB's IgAdependent reduction of experimental asthma [75]. However, in this present study, the beneficial effects of CTB-KDEL wound not be due to the induction of $\operatorname{IgA}$ as demonstrated in the DSS-colitis model using $\mathrm{C} 3 \mathrm{H} / \mathrm{HeJ}$ mice Figure 16. $\mathrm{C} 3 \mathrm{H} / \mathrm{HeJ}$ mice have an intact adaptive immune system, however, lack the proper MHC II haplotype to bind CTB and consequently CTB-KDEL [158]. It has previously been determined that CTB peptide 89-100 is the major T cell epitope of CTB and that $\mathrm{T}$ cell recognition of CTB is dependent on the MHC II context of the host T cells. Therefore, $\mathrm{C} 3 \mathrm{H} / \mathrm{HeJ}$ mice do not produce ADAs or IgA when exposed to CTB or CTB-KDEL (Figure 6). Thus, how predosing of CTB-KDEL was just as effective as one therapeutic dose immediately after DSS exposure remains unknown.

While we know CTB-KDEL induces epithelial restitution in the colon and this is the major contributing function of the proteins therapeutic effect, we cannot discount the role of the immune system in CTB-KDEL's prophylactic effects. It has been demonstrated previously that $\mathrm{CTB}$ can have direct effects on immune system through the binding of GM1 ganglioside on immune cells which results in a immunosuppression [81, 
107], such as Rouqeute-Jazdeanian et al. who showed that the binding of CTB directly to GM1 ganglioside prevented CD4+ T cells activation and proliferation. Additionally, others have shown that non-GM1 binding CTB mutants do not modulate immune function [79]. In support of these results, Coccia et al. determined that daily oral administration of $100 \mu \mathrm{g}$ CTB over a four-day period inhibited mucosal Th1 cell signaling and Th1 cytokine production that resulted in the mitigation of 2,4,6trinitrobenzene sulfonic acid (TNBS)-induced mouse model of Crohn's disease [77]. These findings were corroborated in a in a small multicenter, open-label, and nonrandomized clinical trial in patients with active $C D$, where 7 out 12 patients responded to six $5 \mathrm{mg}$ oral doses of CTB treatment [78] Furthermore, D’Ambrosia et al demonstrated CTB promotes Treg cells by preventing monocyte-derived DC maturation [80] We have also shown that two weeks after two vaccinations with $30 \mu \mathrm{g}$ of CTBKDEL, as performed in figures 4-6, increased Treg cells in the lamina propria while also reducing DSS-induced injury [50]. These effects may not necessarily be a direct effect of CTB-KDEL on immune cells but rather an indirect effect from IECs. Nonetheless, a similar phenomenon has also been reported by others, like Zang et al, who has shown intraperitoneal administration of CTB to rats increased Treg cells in the peripheral blood 24-72 $\mathrm{h}$ after ischemia and decreased neurological dysfunctions and mortality rate [16, 54-58]. Resident Treg cells are known inducers of tissue homeostasis and wound repair, functions that could significantly impact disease parameters in DSS-colitis [175]. It remains to be determined if CTB-KDEL's prophylactic effect is mediated by immune cells or indirectly through IECs remains. Meanwhile, mice vaccinated with CTB-KDEL were greatly protected from DSS induced injury in C57BL/6 and C3H/HeJ mice unlike $\operatorname{Rag}^{-/-}$mice (lacking both T and B cells [159]), where CTB-KDEL demonstrated a 
relatively mild effect [159] (Figure 17). These data suggest an alternative function of CTB-KDEL in the colon mucosa, perhaps related to the adaptive immune system. Nonetheless, how CTB-KDEL's effect persists two weeks after administration remains unknown. Future studies will need to address the impact of CTB-KDEL (and CTB) on the gut immune system of $\mathrm{C} 3 \mathrm{H} / \mathrm{HeJ}$ mice to uncover any alternative immunomodulatory functions of the molecule.

In conclusion, the present study provides evidence for CTB-KDEL's ability to treat chronic colitis. Additionally, we argue that immunogenicity is not a major risk for the mucosal healing activity of orally administered CTB-KDEL. Further research is needed to determine why ADAs did not neutralize or prevent CTB-KDEL's activity and to determine whether this phenomenon is unique to CTB-KDEL or applies to other GItargeted biologics. Nevertheless, collectively these results support the development of CTB-KDEL for UC therapy. 


\section{CHAPTER 5: CTB-KDEL INDUCES A WOUND HEALING RESPONSE IN HUMAN IBD COLON TISSUES 5}

\subsection{Introduction}

As discussed in Chapter 1, CTB-KDEL is a plant-produced recombinant protein derived from cholera toxin B subunit (CTB) [89]. CTB is a non-toxic, pentameric, ganglioside GM1-binding subunit of cholera holotoxin. Recombinantly produced CTB is currently used as an active component in the internationally licensed oral cholera vaccine Dukoral ${ }^{\circledR}$ [106]. Additionally, CTB was shown to exhibit anti-inflammatory activities under various immunopathological conditions $[69,107]$. For example, the airway administration of CTB ameliorated inflammation in an experimental asthma mouse model [75]. In a Phase I/II clinical trial, oral administration of CTB, chemically crosslinked to a peptide from the human $60 \mathrm{kDa}$ heat shock protein, alleviated uveitis of Behcet's disease [49]. Furthermore, oral administration of CTB blunted the intestinal inflammation of CD in a mouse model [77]. In a Phase I clinical trial, approximately $40 \%$ of patients with mild to moderate $\mathrm{CD}$, who received thrice weekly oral administrations of $5 \mathrm{mg}$ of CTB for 2 weeks, showed significantly reduced disease activity indices [78]. These multiple lines of preclinical and clinical in vivo evidence, coupled with the history

\footnotetext{
${ }^{5}$ Modified from: FASEB J. 2019 Sep 27:fj201901255R. doi: 10.1096/fj.201901255R.
} 
of use as an oral cholera vaccine, strongly highlight the potential of CTB and its derivatives as topical biotherapeutics. As mentioned in Chapter 3, the unique active CTB-KDEL in the aforementioned studies are unique to the plant-made variant, as it has a mutation at amino acid position 4 and an ER retention signal sequence at the $\mathrm{C}$ terminus (N4S-CTB-SEKDEL; [89]). The ER-retention sequence was added to CTBKDEL to improve production in planta, while Asn $4 \rightarrow$ Ser mutation was introduced to avoid $N$-glycosylation $[42,89]$. The addition of the KDEL sequence to N4S-CTB significantly reduced ER stress that otherwise caused poor production yield. This modification was critical to achieve high-level accumulation in leaf tissue [41, 42]. Despite the modification, the plant-made CTB variant, CTB-KDEL, is virtually identical to $\mathrm{CTB}$ in regard to GM1-binding affinity, molecular stability and vaccine efficacy to induce anti-toxin IgG and $\operatorname{IgA}$ antibodies upon oral immunization in mice [89]. The protein ER retention mechanism involving the KDEL receptor is highly conserved among eukaryotic organisms [102]. Chapter 3 revealed, CTB-KDEL exhibits unique colon mucosal would healing effects that are mediated by its localization in the ER via KDELR and subsequent activation of a UPR and TGF $\beta$ signaling in colon epithelial cells.

Despite the striking finding described in chapters 3 and 4 the data presented were derived from immortalized cell culture systems and/or mouse models of colitis. The usefulness of nonclinical models is constantly questioned because of the homologous controlled conditions and poor recapitulation of human conditions [176]. Thus, it is imperative to evaluate CTB-KDEL's efficacy in a more clinically relevant model to support its use for IBD therapy. Thus, here we investigated CTB-KDEL's effect on 
inflammation and tissue repair responses in colectomy tissue samples obtained from IBD patients. 


\subsection{Results}

To reveal if the UPR-mediated epithelial cell migration effects in Caco2 cells and epithelial recover activity in mice of CTB-KDEL could translate into a therapeutic effect in human colon tissues ex vivo. A sigmoid colon tissue of a 57-year-old male ulcerative colitis (UC) patient who underwent total colectomy was sectioned into 9 pieces, which were cultured with PBS, CTB-KDEL or CTB ( $n=3$ for each treatment) for $24 \mathrm{~h}$, and then UPR, TGF $\beta$ and wound healing signaling were analyzed by pathway-focused RT-qPCR arrays. Consistent with results observed in Caco2 cells (Figure 6), CTB-KDEL-treated tissue showed a significant upregulation of the IRE1 gene ERN1, PERK gene EIF2AK3 and TGFB1, while there was no significant difference between PBS- and CTB-treated tissues (Figure 18A). Additionally, CTB-KDEL significantly upregulated 21 out of 84 wound healing-related genes in the RT-qPCR array by $>2$ fold compared to PBS, while CTB significantly enhanced only 1 gene (MAPK1; Figure 18B). Of note, one of the 21 genes significantly increased by CTB-KDEL was the E-cadherin gene $C D H 1$, which was also upregulated in the acute DSS model (Figure 10B). Congruent with the RT-qPCR results, histopathological analysis showed that CTB-KDEL-treated tissues $(n=3)$ had early stage crypt formations and relatively low neutrophil infiltration in the mucosa, while PBS- and CTB-treated tissues did not have such distinct histological features (Figure 19C). Since CTB-KDEL-treated tissue was cut adjacent from PBS or CTBtreated ones, these observations likely reflect the effects of treatment. 

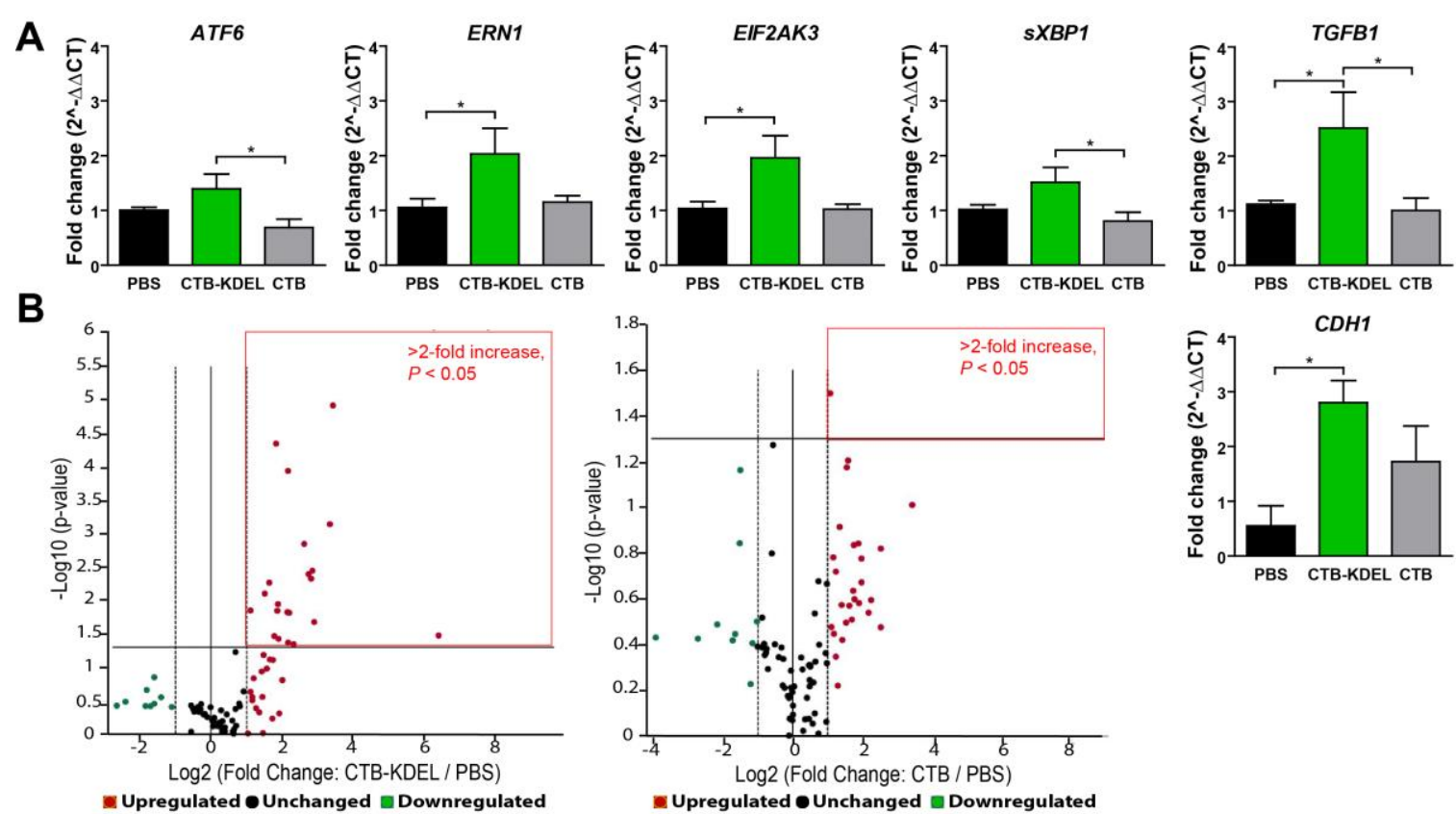

C

Upregulated $\bullet$ Unchanged $₫$ Downregulated
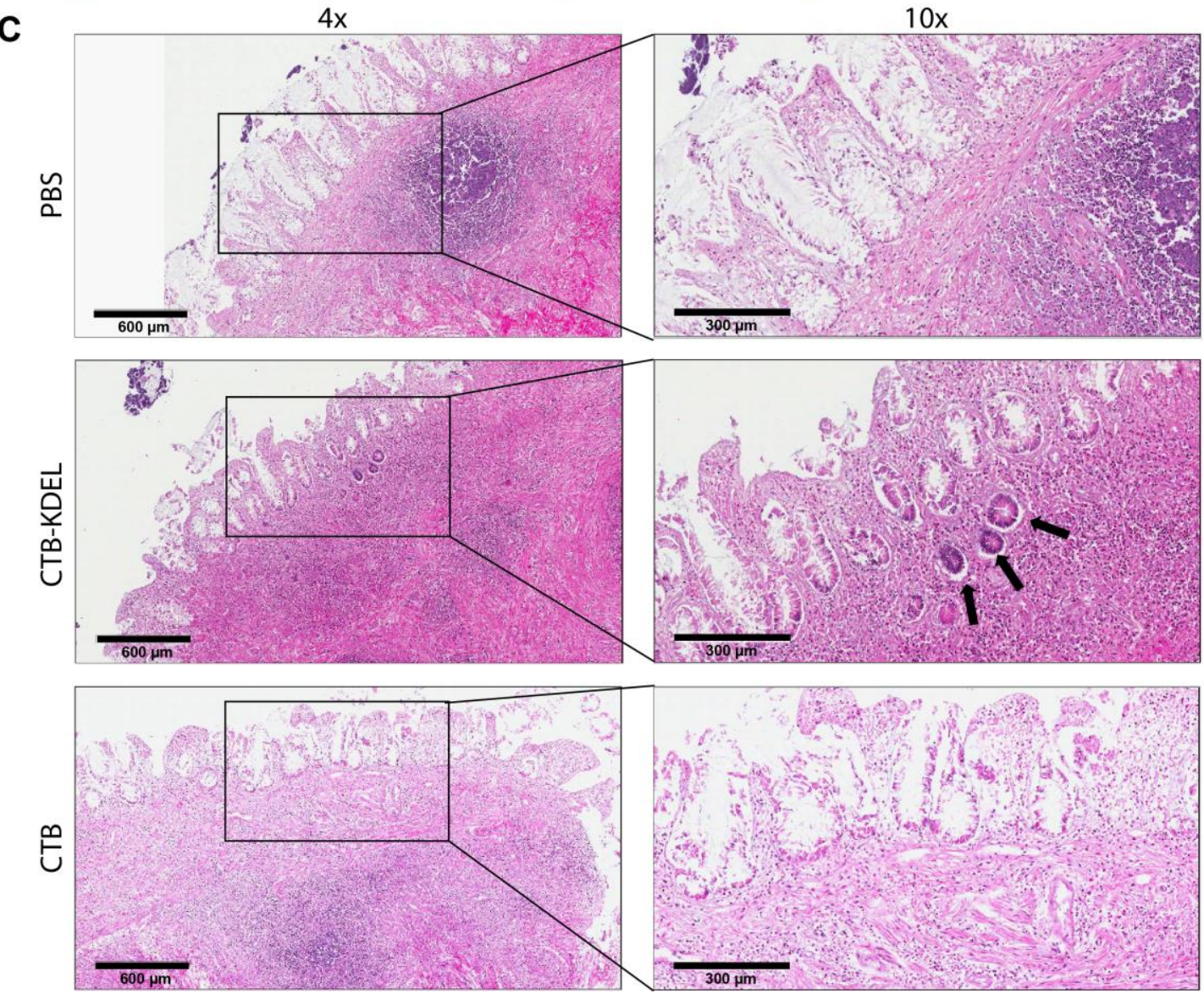

Figure 18. Effects of CTB-KDEL and CTB in human colon tissue isolated from a 57year-old male UC patient who underwent colectomy. CTB-KDEL induces UPR, 
TGF $\beta$ and wound healing signaling in human colon colectomy tissue. The sigmoid colon tissue was divided into nine adjacent sections and cultured with PBS, or $1 \mu \mathrm{M}$ of CTBKDEL or CTB for $24 \mathrm{~h}$. (A) RT-qPCR analysis of UPR and wound healing gene expression. One-way ANOVA with Bonferroni's multiple comparison tests was used to compare all pairs of groups. Mean \pm S.D. is shown for each group $(N=3)$. (B) Wound healing pathway-focused qRT-PCR analysis of gene expression in human UC patient colectomy tissue. Volcano plots show $\mathrm{P}$ value (y axis) and fold-change (x axis) for all 84 genes in the wound healing pathway upon treatment with PBS vs. CTB-KDEL (left) and PBS vs. CTB (right). Dots represent $\geq 2$-fold upregulation (red), $\geq 2$-fold downregulation (green), or $<2$-fold change (black). The area corresponds to $>2$-fold increase with $\mathrm{P}$ values $<0.05$ is highlighted with a red box. Values were calculated by the data analysis web portal at http://www.qiagen.com/geneglobe ( $\mathrm{N}=3$ per treatment). (C) Human UC patient colectomy tissue H\&E stain. Representative 4x (left) and 10x (right) photomicrographs of H\&E stained sigmoid colon tissues from each group. Arrows point to crypt formations.

CTB-KDEL's wound healing effects were further validated in additional IBD colon tissues. Altogether, colectomy tissues from 5 patients were treated with CTBKDEL or vehicle control (PBS) ranging from 20 to 72 years old, including two Crohn's Disease patients (a 57-year-old male and a 60-year-old female). Albeit to varying degrees, CTB-KDEL significantly upregulated multiple wound healing-associated genes by over two-fold in all 5 patients' tissues (Figure 19A). In contrast, there was no gene in the wound healing pathway analyzed that was significantly downregulated by CTBKDEL in any of the tissues (data not shown). The most notable effect was observed in a 
sigmoid colon tissue from a 20-year-old male UC patient, in which CTB-KDEL significantly enhanced as many as 79 out of 84 wound healing-associated genes analyzed (Figure 19A). As observed in the mouse DSS model (Figure 10B), CTB-KDEL significantly increased $C D H 1, T G F B 1$ and WNT5A (Figure 19B). The robust upregulation of wound healing genes was accompanied with distinct crypt formations and relatively low neutrophil infiltration in the mucosa of CTB-KDEL-treated tissues in contrast to those treated with PBS. Ki67 IHC analysis corroborated the histopathological observation, which depicts live, proliferating crypt formation in CTB-KDEL treat tissues $(\mathrm{n}=3)$ (Figure 19C). Taken together, the above results provide evidence that CTB-KDEL can exhibit wound healing activity in human IBD colon tissues, highlighting the protein's therapeutic potential. 


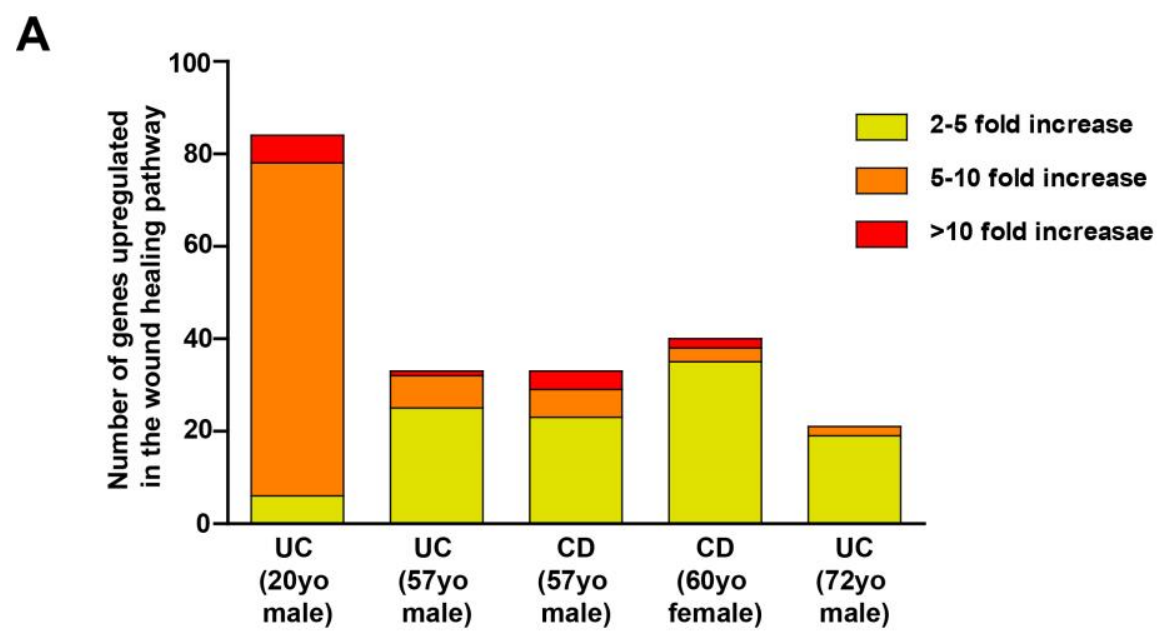

B
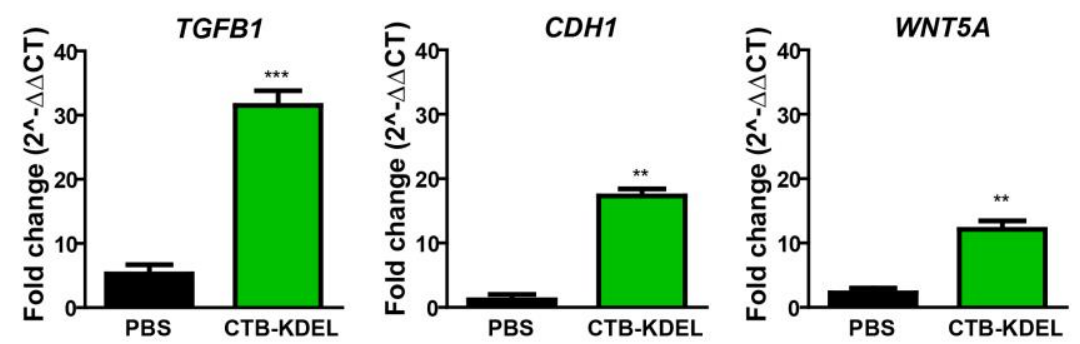

C
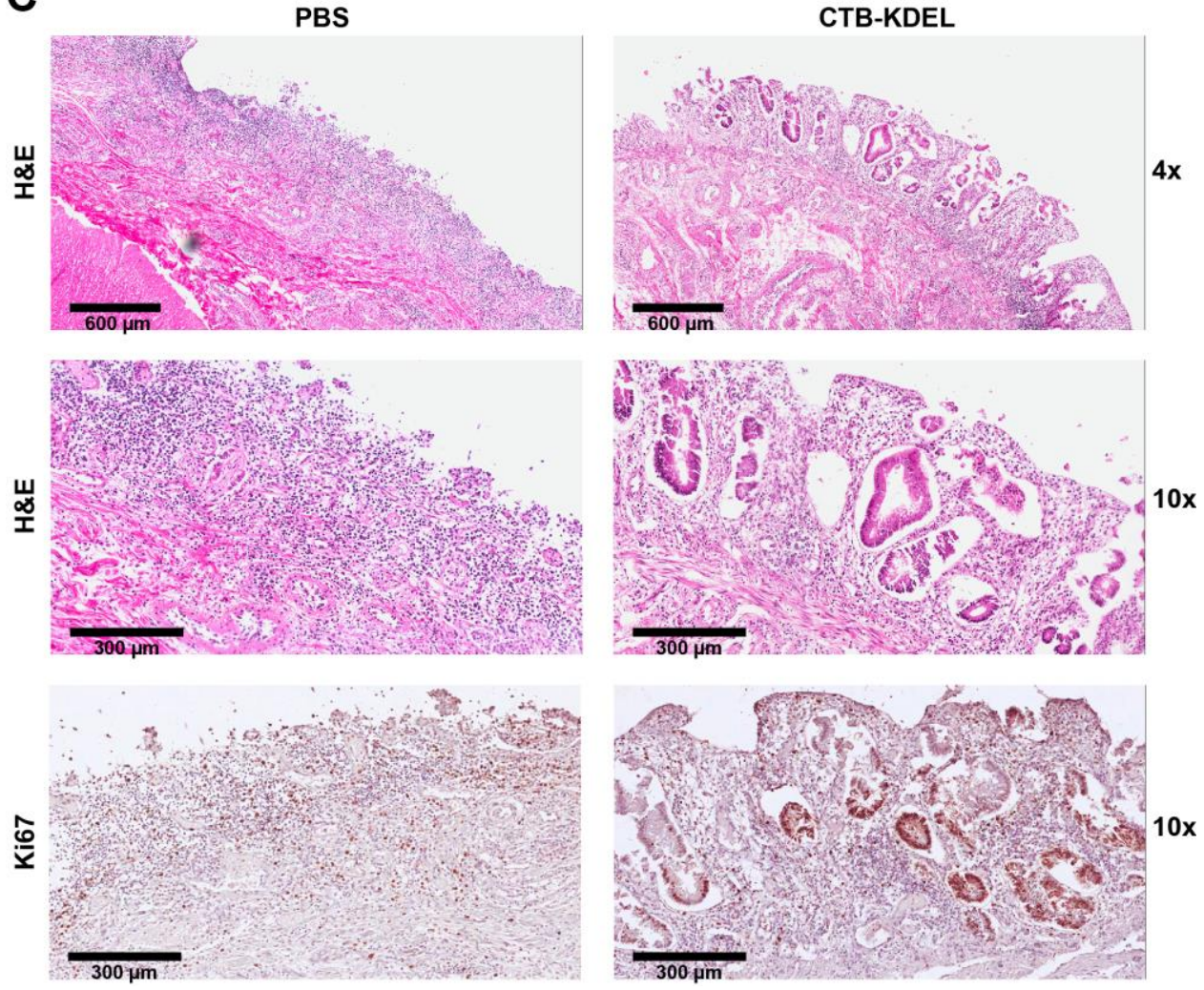
Figure 19. Effects of CTB-KDEL and CTB in human colon tissue from IBD patients who underwent colectomy. (A) Wound healing pathway-focused qRT-PCR analysis of the number of genes upregulated in treated human UC or Crohn's disease (CD) patient colectomy tissues by CTB-KDEL compared to the PBS vehicle control. The graph shows number of gene $\geq 2$-fold upregulated. Values were calculated by the data analysis web portal at http://www.qiagen.com/geneglobe $(\mathrm{N}=3 /$ patient). (B) RT-qPCR analysis of TGFB1, CDH1 and WNT5A gene expression $24 \mathrm{~h}$ after treatment in the tissue from the 20-year-old (20yo) male UC patient. Mean \pm S.D. is shown for each treatment $(\mathrm{N}=3)$. Two-tailed unpaired t-test was used to compare groups. $* *, * * * \mathrm{P}<0.01,0.001$. (C) Representative 4x (left) and 10x (right) H\&E-stained and Ki67 immunohistochemistry photomicrographs of the 20yo male UC patient colectomy tissue. 


\subsection{Discussion}

The data generated from the murine models of DSS colitis presented in Chapters 3 and 4 strongly support the notion that oral administration of CTB-KDEL can facilitate colon epithelial restitution and wound healing, at least in the conditions tested. In corroboration of the acute and chronic DSS models, here we demonstrated the mucosal healing potential of CTB-KDEL in an explant culture model using human IBD patient colectomy tissues (Figure 18 and Figure 19). Although the number of patient colon tissues tested in this study is limited, all five patient specimens showed upregulation of wound healing-related genes in response to CTB-KDEL treatment, in contrast to PBS or CTB. Of note is that, consistent with the findings in the DSS model, CTB-KDEL significantly activated UPR, TGF $\beta$ signaling pathways, $C D H 1$ and WNT5a (Figure 18). Additionally, new crypt formations were observed in CTB-KDEL-treated tissues (Figure 18 and Figure 19). We acknowledge, however, that the wound healing response induced by CTB-KDEL substantially varied among the different patients' tissues tested, for which the underlying mechanism remains unknown. The least wound healing response was observed with the 72-year-old male UC patient, which may be explained by an age effect; recently, it has been reported that aging individuals experience delayed and impaired healing of the gastric mucosa due to reduced angiogenic capacity [177]. Nonetheless, these results overall support the premise that CTB-KDEL could induce mucosal healing in IBD patients, warranting a further study using additional tissue specimens. As there is no cure available for IBD, mucosal healing is currently regarded as the standard treatment goal in IBD therapy $[4,6-8]$. Current therapeutic options can indirectly achieve mucosal healing in only $\sim 50 \%$ of patients and their direct effect on epithelial repair remains elusive $[15,16,178]$. Thus, the results presented herein provide implications for the 
unique therapeutic potential of CTB-KDEL that may address a significant unmet need in UC treatment.

In conclusion, the data herein reveal that the mechanism of action of CTB-KDEL demonstrated in nonclinical studies also induces mucosal wound healing pathways and observable histological improvements in the colectomy tissues of IBD patients treated ex vivo. These data provide the proof of concept of CTB-KDEL's remarkable efficacy and provide justification for its use in a first-in-human Phase I trial to support its development as a new agent for the treatment of inflammatory bowel disease. 


\section{CHAPTER 6: SUMMARY AND IMPLICATIONS FOR FUTURE DIRECTIONS}

\subsection{Summary}

Although there is currently no curative therapy available for UC, the recent advent of anti-TNF $\alpha$ agents has revealed that mucosal healing provides an important clinical endpoint in UC treatment, as it is closely associated with sustained clinical remission, improved quality of life and fewer surgical operations and cancer incidence [4-9]. The development of new drug candidates for mucosal healing represent a significant unmet need in UC management. However, currently there is no mucosal wound repair product indicated for UC available or in clinical trials (ClinicalTrials.gov, as of August 28, 2019). Previously, we have proposed that a modified CTB containing an artificial ER retention motif (CTB-KDEL) could be used to facilitate mucosal healing in the management of UC. Here, we determined the mechanisms by which CTB-KDEL induces colon epithelial mucosal healing. This effect was revealed in a series of experiments where we first determined in a Caco2 cell wound-healing model that variants of CTB-KDEL that lack the terminal KDEL sequence (CTB and CTB-KDE) failed to induce cell migration in Caco2 cells Figure 2. It was further determined that CTB-KDEL colocalized with KDELR and remained longer than CTB within the ER of Caco2 cells (Figure 4 and Figure 5). This prolonged retention of CTB-KDEL increased the levels of UPR sensors by 1.5 - 2-fold in Caco 2 and mouse primary colon epithelial cells, as well 
as in a human UC colon tissue (Figure 6, Figure 8, and Figure 18) Subsequently, genetic and chemical inhibition of IRE1-XBP1 signaling completely abolished the TGF $\beta$ upregulation and cell migration effects of CTB-KDEL in Caco2 cells (Figure 7). Combined with our previous data showing that GM1 binding is essential for CTBKDEL's wound healing effect in Caco2 cells [50], these data strongly suggest that the Cterminal KDEL sequence rendered CTB-KDEL with a unique new wound healing activity in the colon epithelial cells via localization to the ER, UPR activation and subsequent TGF $\beta$ signaling.

CTB-KDEL's capacity to induce a UPR and epithelial restitution/wound healing was corroborated in a DSS-induced acute colitis mouse model where a single oral administration of as little as $3 \mu \mathrm{g}$ of CTB-KDEL significantly enhanced recovery from colitis (Figure 9 and Figure 10). The therapeutic effects were characterized by enhanced epithelial regeneration, demonstrated by E-cadherin IHC and upregulated $C d h 1$ and Wnt5a levels, suggesting that the rapid resolution of epithelial injury is the primary mechanism of CTB-KDEL's efficacy in this model Figure 10. Due to the fact that acute DSS-induced colitis typically only incorporates features of innate immunity and acute epithelial injury, Chapter 4 addressed the therapeutic potential of CTB-KDEL in a chronic progressive model of colitis. We implemented an established model of chronic colitis (Figure 11) by giving repeated DSS exposures to mice that is known to create a complex interplay between innate immune responses and compromised epithelial barrier function that drives a chronic sustained inflammatory response $[96,149,150]$. Subsequently, we found that weekly oral administration of CTB-KDEL dosed before or after the onset of chronic conditions induced by repeated DSS exposure, could significantly reduce DAI scores, intestinal permeability and pro-inflammatory cytokines 
(Figure 12). The therapeutic efficacy of CTB-KDEL against chronic colitis was corroborated by histological evidence for significant remission and healing in CTBKDEL treated mice. In addition, it was also noted that there was minimal neutrophil infiltration in tissues of CTB-KDEL treated mice in contrast to vehicle control (Figure 13). The data also revealed that $\mathrm{QW}^{\mathrm{d} 7}$ and $\mathrm{Q} 3 \mathrm{D}^{\mathrm{d} 35}$ dosing of CTB-KDEL were more efficacious than $\mathrm{QW} \mathrm{W}^{\mathrm{d} 35}$ dosing (Figure 12). This provides a basis for both $\mathrm{QW}$ and

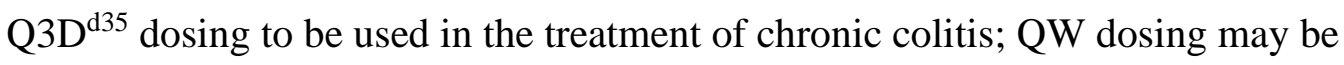
sufficient as a maintenance dose to prevent relapse of disease, while Q3D ${ }^{\mathrm{d} 35}$ dosing may be better suited for treatment of active disease.

Moreover, we have previously shown that oral administrations of 3, 10, or $30 \mu \mathrm{g}$ CTB-KDEL twice at a two-week interval induced anti-CTB-KDEL neutralizing antibodies [89]. However, Figure 12 and Figure 13 demonstrate that CTB-KDEL did not lose its efficacy with repeated dosing (8 doses total $\left.\mathrm{QW}^{\mathrm{d} 7}\right)$. A key pharmacology and toxicology question for biotherapeutic development is immunogenicity; specifically, ADAs. A major disadvantage of therapeutic proteins is that almost all induce an antibody response [169]. ADAs constitute a theoretical risk because they may lead to a loss of efficacy, altered pharmacokinetics (PK), and potentially cause immunotoxicity $[152,153$, 170, 171]. Thus, to address the consequences of immunogenicity, mice (C57BL/6, C3H, or $\operatorname{Rag} 1^{-/-}$strains) were pre-exposed to CTB-KDEL, and then subjected to DSS colitis and CTB-KDEL treatment (Figure 14). While the pre-dosing of CTB-KDEL indeed elicited high-titer anti-drug antibodies (ADAs) of the IgA isotype in the intestine of C57BL/6 mice, the therapeutic effects of CTB-KDEL were similar and better than those observed in $\mathrm{C} 3 \mathrm{H}$ and $\operatorname{Ragl}^{-/-}$mice, respectively, both of which showed few ADAs under the same experimental conditions (Figure 15, Figure 16, and Figure 17). Thus, the 
immunogenicity of CTB-KDEL does not seem to impede the protein's mucosal healing efficacy. These data argue that immunogenicity is not a major risk for the mucosal healing activity of orally administered CTB-KDEL. However, further research is needed to determine why ADAs do not neutralize or prevent CTB-KDEL's activity and to determine if this phenomenon is unique to CTB-KDEL or applies to other GI-targeted biologics.

Lastly, to address if CTB-KDEL could induce mucosal healing in IBD patients, we determined the mucosal healing potential of CTB-KDEL in an explant culture model using human IBD patient colectomy tissues. In these studies, CTB-KDEL induced an UPR, upregulated wound healing pathways and maintained viable crypts in colon explants from IBD patients (Figure 18 and Figure 19). These data revealed that the mechanism of action of CTB-KDEL demonstrated in nonclinical studies also induces mucosal wound healing pathways in the colectomy tissues of IBD patients treated $e x$ vivo. These data provide the proof of concept of CTB-KDEL's efficacy and provide justification for its use in a first-in-human Phase I trial to support its development as a new agent for the treatment of IBD.

\subsection{Future Directions}

Herein our in vivo and ex vivo studies revealed how CTB-KDEL elicits its unique therapeutic effects in colitis. [36-39, 51, 52] It was determined that there are at least three necessary components of CTB-KDEL's mechanism of action (MOA) that are essential for CTB-KDEL's TGF $\beta$-mediated would healing effect [50]. First, endocytosis into cells via GM1 ganglioside binding; second, induction of an UPR mediated by the prolonged retention of CTB-KDEL in the ER by the KDELR; and lastly, induction of TGF $\beta$ 
mediated by IRE1/XBP1 signaling. While we have identified these three essential checkpoints in CTB-KDEL's MOA, there remains unanswered questions around each molecular phenomenon.

First, CTB-KDEL MOA is initiated by the binding of GM1 ganglioside that allows CTB-KDEL to be endocytosed into the cell. While this is not the only route of CTB into cells, we have proved it is essential for CTB-KDEL's therapeutic effect (Figure 20). One might speculate that other KDEL tagged proteins could also induce a similar effect. However, the data presented in Figure 20 and Figure 21 suggest that GM1 may critical roles in epithelial repair induced by CTB-KDEL (discussed below).

CTB is known to bind to glucosylated proteins, with a superior affinity for the branched pentasaccharide moiety of ganglioside GM1 [179]. Previous studies have shown that CTB can bind more than just GM1 as demonstrated by fluorescence-activated cell sorting of mouse embryonic neuroepithelial cells treated with CTB that revealed CTB binding did not coincide with GM1 expression levels, suggesting alternative receptors are present [180]. Alternative receptors with affinity for CTB, although much lower than GM1, have been identified, many of which are GM1 ganglioside-like receptors such as GM2 and GD1a or glycoprotein type receptors [37, 38, 181, 182]. In line with these findings we found that CTB-KDEL and the non-GM1 binding G33D-CTBKDEL (Figure 20) both bind to Caco2-cells, albeit G33D-CTB-KDEL's binding to Caco-2-cells was much weaker than CTB-KDEL's, and co-localized with the KDELR in Caco2-cells (Figure 21). However, only CTB-KDEL induced an UPR, increased TGF $\beta$ levels, and subsequent wound healing (Figure 6, Figure 8, \& Figure 20). These data suggest that GM1-ganslioside plays more of a role in CTB-KDEL MOA other than just cell entry. We speculate that the cell-surface GM1-ganglioside that is brought to the ER 
as a complex with CTB-KDEL may be primarily responsible for UPR activation.

Previously, Tessitore et al demonstrated that accumulation of GM1-ganglioside in the ER resulted in an UPR that was characterized by an upregulation of ER chaperones, such as BiP, and XBP1 activation [183]. These effects were attributed to GM1-induced calcium depletion in the ER because of a reduction in the sarcoplasmic/ER calcium ATPase activity, which is responsible for calcium uptake into the ER [184]. These results were later corroborated by Sano et al. who revealed that GM1-ganglioside accumulates in a glycosphingolipid-enriched microdomain of the mitochondria-associated ER membranes [185]. In these studies, excess GM1-accumulation in the ER was shown to induce $\mathrm{Ca}^{2+}$ flux from the ER to mitochondria and a UPR, which resulted in ER stress- and mitochondria-mediated apoptosis of neuronal cells. However, CTB-KDEL may not cause such excess accumulation of the ganglioside in the ER and a cytotoxic UPR response, as we did not observe any cytotoxic response to CTB-KDEL in Caco2 cells (Figure 2). In our preliminary study using polarized T84 cell monolayers, we found that the levels of UPR marker genes induced by CTB-KDEL were significantly lower than the well-known UPR inducer thapsigargin (data not shown). We postulate that the amount of cell-surface GM1 ganglioside sequested by CTB-KDEL was a limiting factor. Alternatively, the availability of KDELR was limited, whereby the receptor became saturated before the concentration of CTB-KDEL reached a point that overload GM1 in the ER.

Meanwhile, the data in Figure 6 showed that CTB-KDEL can directly interact with BiP. Therefore, a question remains whether the accumulation of GM1 (via complex with CTB-KDEL) in the ER is sufficient to induce IRE1-XBP1 signaling or the binding of CTB-KDEL to BiP also plays a role in the initiation of UPR. Additionally, it remains 
to be determined if CTB-KDEL also directly interacts with IRE1 to displace BiP since IRE1 signaling can be activated by the binding of proteins (unfolded) to its peptide binding groove after the release of BiP [186-189]. Future research should determine the relative contributions of GM1 and CTB-KDEL to epithelial repair activity and how exactly the UPR is activated for subsequent TGF $\beta$ upregulation. 


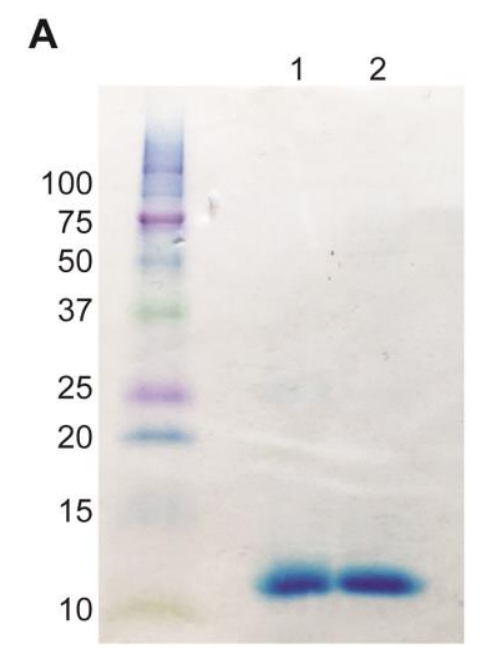

B
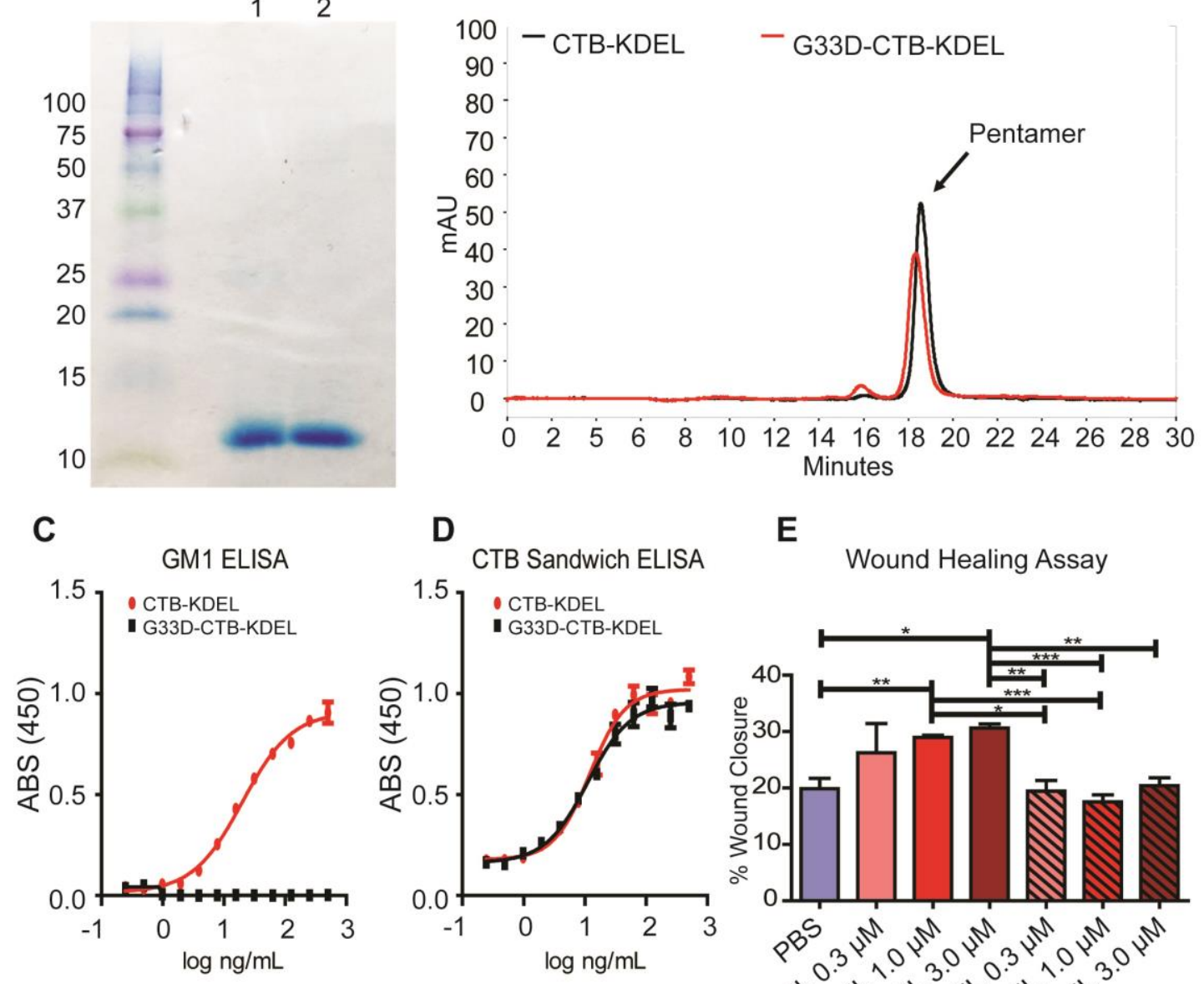

E Wound Healing Assay
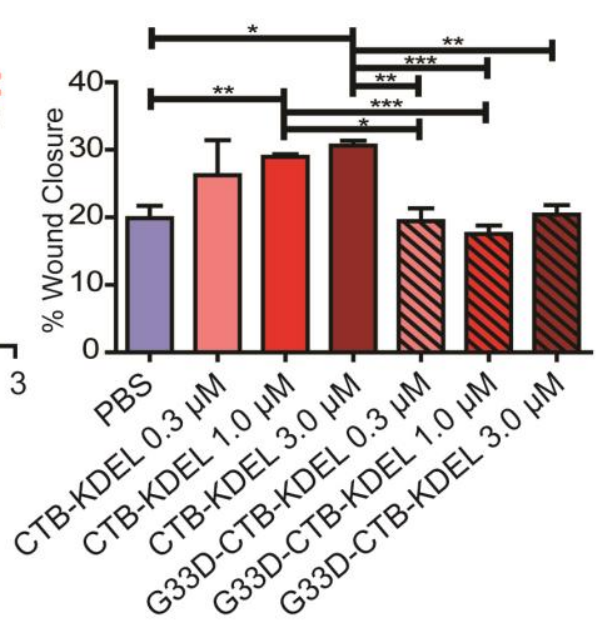

Figure 20. Characterization of G33D-CTB-KDEL. Nucleotide G at position 161 was mutated to an A (for Gly33 to Asp CTBp), creating pNM175. G33D-CTB-KDEL was expressed and purified as previously described (10). (a) SDS PAGE analysis. $10 \mathrm{ug}$ of purified CTB-KDEL (lane 2) and G33D-CTB-KDEL (lane 1) appeared as monomers under heat denaturing conditions. (b) SF-HPLC analysis. Chromatography was performed on a Beckman Coulter System Gold HPLC. CTBp or G33D-CTB-KDEL was applied to an SEC column and elution was monitored by absorbance at $280 \mathrm{~nm}$. The large 
peak corresponds to pentamers of CTB purified to $>95 \%$ homogeneity; the minor peak is likely decameric [Yasuda et al. 1998]. (c) GM1 ganglioside ELISA was performed as previously described [10]. G33D-CTBp showed no affinity towards GM1 ganglioside, samples were ran in duplicate and analyzed by GraphPad Prism 5.0. (d) CTB sandwich ELISA where CTBp and G33D-CTBp were captured by rat anti-CTB mAb and detected by goat anti-CTB. No significant difference was observed between either CTB protein in the binding affinities of anti-CTB antibodies, samples were run in duplicate and analyzed by GraphPad Prism 5.0. (e) Wound Healing Assay. Analysis of in vitro wound closure after $24 \mathrm{~h}$ by wound area measurement. G33D-CTB-KDEL does not enhance wound healing. Means \pm SEM of four independent experiments are shown. $* \mathrm{P}<0.05$, $* * \mathrm{P}<$ 0.01, and ***P, 0.001; one-way ANOVA with Bonferroni's multiple comparison tests. (Baldauf and Royal et al. Muco Imm 2017)

The second essential component of CTB-KDEL's MOA is the ability to bind to the KDELR. In a Caco2 cell wound-healing model, CTB-KDEL, but not CTB or CTBKDE, facilitated cell migration via interaction with the KDELR, localization in the ER, UPR activation and subsequent TGF $\beta$ signaling. Since native CTB lacking an ER retention motif also has the ability to complex with GM1 and traverse through the endomembrane system, it is clear that KDELR plays a critical role that allows CTBKDEL and/or GM1 to trigger an UPR in the ER for a subsequent epithelial repair response. Inhibition of the inositol-requiring enzyme 1 (IRE1)/X-box binding protein 1 (XBP1) arm of UPR abolished the cell migration effect of CTB-KDEL, indicating that the pathway is indispensable for the activity. Here the question remains how CTB-KDEL 
induces IRE1/XBP1 pathway. The data in Figure 6 suggests that the binding of CTBKDEL to BiP plays a role in the initiation of UPR seen with CTB-KDEL treatment.

What is unknown is if CTB-KDEL also directly interacts with IRE1 to displace BiP or if the binding of the KDELR alone is sufficient to induce BiP dissociated from IRE1 and subsequent downstream signaling. There is also a possibility that the UPR response is in part attributed to competition between CTB-KDEL and BiP for KDELR, as BiP relies on KDELR for retrieval back to the ER [121, 190-192]. In this regard, the role of KDELR in CTB-KDEL-induced epithelial repair activity warrants further investigation.

A

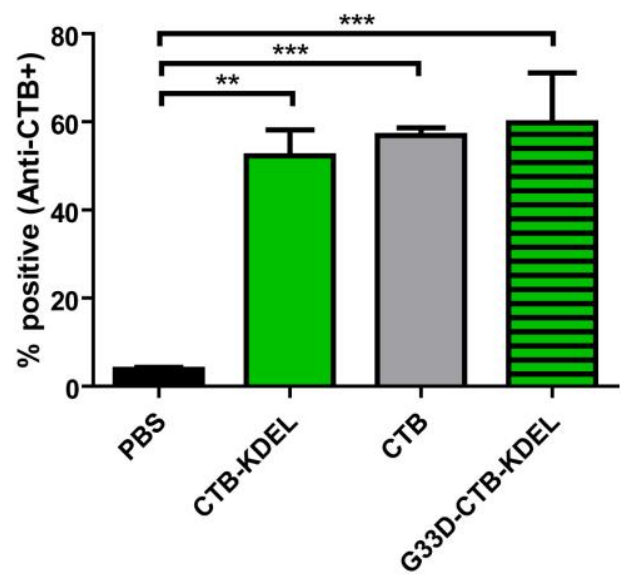

C

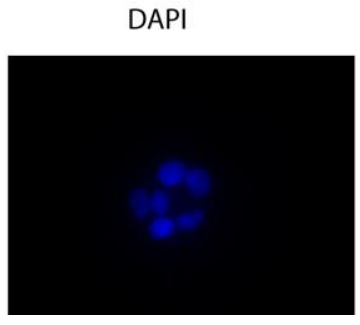

Anti-KDELR

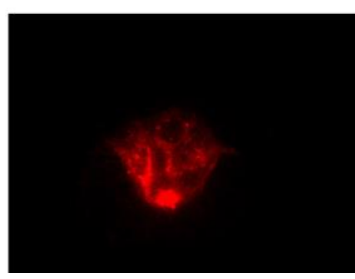

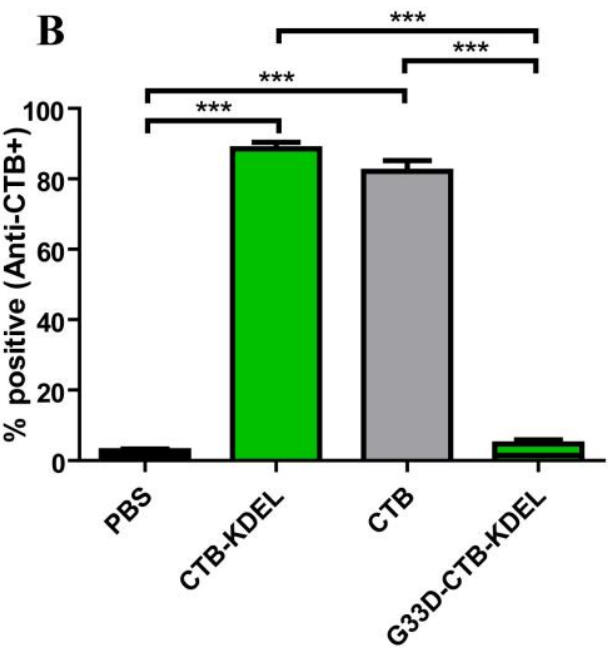

Anti-CTB

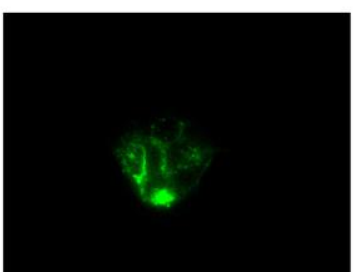

Merge

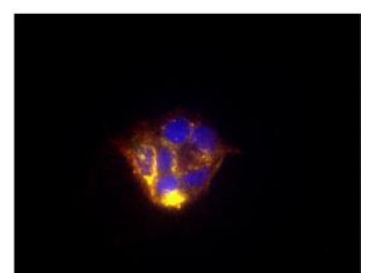

Figure 21. G33D-CTB-KDEL loosely binds to Caco2 cells and localizes with the

KDEL receptor (KDELR). Flow cytometry analysis of Caco2 cells incubated with CTB-KDEL, CTB, or G33D-CTB-KDEL at room temperature (A) or (B) on ice. (A) 
Caco2 cells were treated with $1 \mu \mathrm{M}$ CTB-KDEL, CTB, or G33D-CTB-KDEL at room temperature for 15 minutes then fixed and stained with anti-CTB labeled Alexa Fluor 488 antibody then analyzed by Flow cytometry $(\mathrm{n}=4)$. (B) Caco2 cells were treated with $1 \mu \mathrm{M}$ CTB-KDEL, CTB, or G33D-CTB-KDEL on ice for 15 minutes then fixed and stained with anti-CTB labeled Alexa Fluor 488 antibody then analyzed by Flow cytometry ( $\mathrm{n}=$ 4). (C) Immunofluorescence analysis of G33D-CTB-KDEL intracellular localization within the ER. Caco2 Cells were treated with $1 \mu \mathrm{M}$ of G33D-CTB-KDEL, or a vehicle control (PBS) for $24 \mathrm{~h}$. Cells were fixed/permeabilized and stained with an anti-CTB monoclonal antibody detected by Alexa FluorTM 488 (green), Anti-KDEL polyclonal antibodies (red), and DAPI (blue). See Figure 5 for vehicle control, CTB, and CTBKDEL corresponding data.

The third essential component of CTB-KDEL's MOA is the induction of TGF $\beta$. CTB-KDEL's epithelial repair activity seems to be mediated by TGF $\beta$, as an anti-TGF $\beta$ antibody and small molecule inhibitor of TGF $\beta$ intracellular signaling (SB-431542 [193]; data not shown) completely inhibited the CTB-KDEL's cell migration effect in Caco2 cells [50]. CTB-KDEL's ability to regulate TGF $\beta$ is mediated through IRE1/XBP1 signaling, as demonstrated in Figure 7. However, how IRE1/XBP1 signaling leads to TGF $\beta$ upregulation is still unknown. This phenomenon has been studied by Chusri et al. in the context of increased TGF $\beta$ levels in hepatitis C virus (HCV) infected hepatocytes [132]. The authors demonstrated that all three arms of the UPR were activated in $\mathrm{HCV}$-infected liver cells, which was responsible for the increased TGF $\beta$ levels. Interestingly, it was determined that $\mathrm{HCV}$ infection led to $\mathrm{NFkB}$ activation through the IRE1/XBP1 pathway and that inhibition of this pathway reduced HCV- 
induced TGF $\beta$ elevation. Thus, it is possible that CTB-KDEL also activates NFKB following UPR stimulation. However, $\mathrm{NF \kappa B}$ regulates numerous cellular pathways as well as the expression of a variety of proteins besides TGF $\beta$ that inhibit apoptosis, regulate the immune system, promote cell survival/proliferation. Additionally, NFkB is implicated in carcinogenesis [194, 195]. NFאB signaling can be broken down into two pathways, canonical and non-canonical. The canonical NFkB pathway is associated with cell survival responses, cellular proliferation, and/or inflammatory signaling, while the non-canonical pathway leads to lymphoid organogenesis, and the production of chemokines and cytokines. Both pathways have distinct nuclear factors that are activated during signaling, such as IкB $\alpha$ for the canonical pathway, while the non-canonical pathway activates the RelB/p52 NF- $\mathrm{kB}$ complex that relies on the processing of p100 instead of degradation of I $\mathrm{KB} \alpha$ [196]. Thus, a stepwise approach to first determining which NFkB pathway is activated by CTB-KDEL may prove to be insightful in determining how or if the protein utilizes the transcription factor.

While determining how CTB-KDEL enhances TGF $\beta$ levels may help identify a new drug target, it is also of high priority to investigate the consequences of TGF $\beta$ activation on the GI tract. TGF $\beta$ is a pleiotropic cytokine playing critical roles in cell differentiation and proliferation, as well as dynamic biological processes in wound healing and immune responses $[23,197,198]$. The cytokine is also involved in various pathological conditions. For example, elevated TGF $\beta$ levels have been correlated to the development of fibrosis following injury to the skin [199]. TGF $\beta$ mediates epithelial-tomesenchymal transition (EMT) [200], and reduction of TGF $\beta 1$ levels in a mouse model of pulmonary fibrosis blunted fibrosis [201]. TGF $\beta$ signaling also has important implications in cancer. Although the cytokine functions as a suppressor of tumorigenesis at an early 
stage of tumor development, its expression is correlated with tumor progression and poor prognosis at late stages $[23,202]$. Collectively, the two-sided nature of TGF $\beta$ points to the importance of careful investigation of possible consequences upon long-term CTB dosing for the treatment of chronic inflammatory diseases. As mentioned in the Introduction section, CTB-KDEL treatment significantly mitigated gut inflammation and reduced tumor development in a model of CAC [50], providing a basis for further investigations of the effects of long-term therapeutic use of CTB-KDEL for the treatment of IBD.

As discussed above, we have discovered that CTB-KDEL utilizes a unique mechanism to induce increased epithelial cell TGF $\beta$ signaling and subsequent migration towards wound healing. However, the potential impacts of CTB-KDEL on other cell types remain largely unknown. GM1 ganglioside is broadly found on various cell types such as epithelial cells, macrophages, dendritic cells (DCs), B cells, T cells, and neurons [36-39]. But outside of what have been presented in this dissertation, the research of the effects of CTB or CTB-KDEL on different cell type has focused on the presence of GM1 ganglioside on the luminal surface of APCs. This is due to CTB's strong mucosal immunostimulatory effects associated with MHC class II expression and local antigen enrichment [40]. For instance, in a mouse model of asthma, CTB suppressed the ability of DCs to prime for Th2 responses to an inhaled allergen and caused a TGF $\beta$-dependent increase in antigen-specific IgA in the airway luminal secretion. The induction of IgA appeared to be essential as CTB's therapeutic effects were abrogated in mice lacking luminal IgA transporter (polymeric Ig receptor) [75]. In a 4, 6-trinitrobenzenesulfonic acid (TNBS)-induced acute colitis mouse model, CTB administration reduced IL-12 and IFN $\gamma$ secretion, inhibited STAT-4 and STAT-1 activation and downregulated T-bet expression, indicating that CTB inhibited mucosal Th1 cell signaling [77]. In vitro, CTB 
decreased monocyte-derived DC maturation and IL-12 production upon lipopolysaccharide (LPS) stimulation in vitro. Moreover, CTB-pretreated, LPSstimulated DCs induced low proliferating T cells that had enhanced production of IL-10 and reduced production of IFN $\gamma$ [80].

It remains to be determined whether CTB-KDEL exhibits any immunomodulatory effect on immune cells like CTB. We do know, however, vaccination with CTB-KDEL (as shown in Figure 14) significantly increased Th2, Treg and Th17 cells and decreased B cells in the lamina propria [50] . Thus, there is evidence suggesting that CTB-KDEL modulates $\mathrm{B}$ and $\mathrm{T}$ cells, although the consequences and underlying mechanism remain unknown. As shown in Chapter 4, CTB-KDEL showed both prophylactic and therapeutic effects against mitigated DSS colitis in $\mathrm{C} 3 \mathrm{H}$ mice. However, in this study, the effects would not be due to CTB-KDEL's capability to stimulate a classic adaptive immune response since $\mathrm{C} 3 \mathrm{H}$ mice lacks the proper $\mathrm{MHC}$ II haplotype to bind $\mathrm{CTB}$ and consequently CTB-KDEL [158]. In fact, we observed a very low, if any, anti-CTB IgA in these mice (Figure X). Since the prophylactic effect of CTB-KDEL was not seen in RAG1 KO mice, these data suggest an alternative function of CTB-KDEL in the colon mucosa, perhaps related to the direct binding of CTB-KDEL to GM1-ganglioside on adaptive immune cells. Future studies will need to address the impact of CTB-KDEL (and $\mathrm{CTB}$ ) on the immune cells of $\mathrm{C} 3 \mathrm{H}$ mice to uncover any alternative immunomodulatory functions of the molecule.

Even though CTB first encounters IECs upon oral administration, the CTBmediated modulation of IECs and the consequence of IEC modulation on the mucosal immune system have largely been ignored in comparison to the protein's direct impacts on immune cells. Previously, we have shown that CTB-KDEL alters the levels of 
inflammatory and wound healing-related cytokines secreted by the colon epithelial cellline Caco2, hinting that CTB-KDEL could induce epithelial cell-mediated immune modulation [50]. Additionally, we have shown that oral administration of CTB-KDEL in mice had pronounced impacts on the distal GI tract, whereby TGF $\beta$-mediated pathways were significantly activated in the colon mucosa along with increase in innate immune cell populations including DCs, natural killer (NK) cells and macrophages (both M1 and M2) in the colon lamina propria [50]. Given that there is a strong link between epithelialderived TGF $\beta$ and innate immune cells in wound healing [18, 92-94], future research should also address the impact of CTB-KDEL on the innate immune cells of the mucosa as well as different cell types lining the epithelium, such as crypt-base stem cells, goblet and endocrine cells and enterocytes [203], as GM1 is widely expressed on the surface of a wide variety of cell types.

Chapters 3 and 4 addressed the ability of CTB-KDEL to mitigate acute colitis, prevent chronic colitis, and resolve chronic colitis symptoms. While CTB-KDEL was efficacious in all scenarios, additional models of IBD should be implemented to validate the therapeutic potential of this molecule. IBD is a heterogeneous disorder that encompasses various phenotypic subtypes and clinical appearances. There is no single animal model that incorporates all aspects of human IBD. Additionally, no two animal models of IBD are pathologically the same, but the multiple models of IBD together can replicate many of the aspects of human IBD subtypes. For example, when DSS is administered to mice, as used in Chapters 3 and 4, it causes a disruption of the intestinal epithelial barrier that allows the entry of luminal bacteria and bacterial antigens into the mucosa that results in an immune response [95]. The immune response leads to inflammation limited to the colon and subsequent colonic mucosal damage that is 
characterized by ulcers/erosions, loss of crypts, and infiltration of granulocytes. Many studies have performed DSS-colitis experiments using animals that have a variety of genetic deficiencies in permeability [204-206] and have revealed that some of these mice are more susceptible to DSS colitis. In line with these data, drugs that reduce gut permeability or maintain barrier integrity are protective against DSS-induced damage [160, 206, 207]. Therefore, since CTB-KDEL induces epithelial restitution and wound repair, it is compelling that the protein can effectively mitigate DSS-colitis, as presented in chapters 3 and 4. As shown in Chapter 4, Rag $1^{-/}$mice developed colitis when given DSS despite lacking $\mathrm{T}$ and $\mathrm{B}$ cells, indicating that the inflammation and damage were driven by the innate immune system [95]. Therefore, while DSS-induced colitis is a great model to assess gut integrity, mucosal responses to gut microflora and innate immune functions, it does not completely mimic human IBD, as vast epithelial cell damage and extensive invasion of the lamina propria by bacteria and bacterial antigens is unlikely to be a principal mechanism of human IBD [160]. Additionally, human IBD is characterized by polarized $\mathrm{T}$ cell responses, whereas the chronic inflammation induced by DSS exposure is characterized by a mixed Th1 and Th2 response [95, 149].

Unlike DSS-induce colitis, the haptenating agent TNBS induces T cell mediated inflammation and subsequent intestinal damage. Intrarectal administration of TNBS results in hapteniztion of the colonic proteins and thereby initiates an mucosal Th1 cellmediated immune response and characterized by infiltration of the lamina propria with CD4+ T cells, neutrophils, and macrophages as well as development of severe diarrhea, weight loss, and rectal prolapse. [95, 149]. TNBS colitis has been widely used in the study of immunologic aspects relevant to Crohn's disease, as many of the characteristics of TNBS colitis, including transmural inflammation and IL-12/INF $\gamma$ cytokine secretion 
patterns, resemble features of Crohn's disease [95, 149, 160]. Interestingly, the TNBS colitis model has greatly contributed to our understanding of CTB's ability to mitigate inflammation. Boirivant et al implement a TNBS-colitis model where daily oral administration of CTB after the onset of TNBS-colitis immediately resolved weight loss and reduced inflammation [70]. As discussed previously, CTB administration also reduced IL-12 and IFN $\gamma$ secretion, inhibited STAT-4 and STAT-1 activation, and downregulated T-bet expression, indicating that CTB inhibited mucosal Th1 cell signaling [77]. It should be noted that CTB failed to prevent DSS-colitis in our study, which is in sharp contrast to the findings by Boirivant et al. Although a direct comparison to the therapeutic effects induced by CTB-KDEL cannot be made due to two different animals models of IBD, the discrepancy in CTB's efficacies could be in part explained by the different dose amounts and timings employed in those two studies; whereas Boirivant et al. administered 4 daily doses of $100 \mu \mathrm{g}$ CTB immediately after the administration of TNBS [70,77], we dosed one $3 \mu \mathrm{g}$ dose of CTB-KDEL or CTB at the end of DSS exposure. This points to the possibility that the low dose of CTB was not sufficient to show a therapeutic effect in the DSS model. Conversely, whether CTB-KDEL at high or low dose levels exhibits T cell-inhibitory effects like CTB remains to be determined. In addition to adding to our understanding of CTB's ability to mitigate inflammation, the TNBS-colitis model has also contributed to our understanding that intestinal inflammation can be abrogated by oral tolerance. Previously, is has been reported that oral administered haptenized colonic proteins $(\mathrm{HCP})$ before rectal administration of TNBS effectively suppressed TNBS-induced colitis [208]. Of note, the suppression was shown to be due to the generation of mucosal Treg cells that produced TGF $\beta$ and a Th2 cells response. Additionally, CD4+ T cells isolated from the lamina 
propria of HCP-fed animals had a 5 to 10 -fold increase in their production of TGF $\beta$. Concomitant systemic administration of anti-TGF $\beta$ diminished the protective effects seen in HCP-fed animals. Thus, the report was the first to show that TGF $\beta$ induction can help prevent and/or resolve experimental TNBS-colitis, even after disease was established. Furthermore, these data were also the first to implicate the regulation of TGF $\beta$ levels in the treatment of IBD [208]. It would be of great interest to test the ability of CTB-KDEL to mitigate TNBS-colitis in future research. As CTB-KDEL was shown to induce TGF $\beta$ signaling in the colon (Figure 18) and a wound healing response in colonic explants from Crohn's disease patients (Figure 19), the TNBS model could further reveal the therapeutic potential of CTB-KDEL in Crohn's disease. 


\subsection{Conclusion}

The data herein revealed that CTB-KDEL exhibits unique colon mucosal would healing effects, which are at least in part mediated by its localization to the ER and subsequent activation of IRE1-XBP1 signaling in colon epithelial cells. The studies presented herein strongly suggest CTB-KDEL's potential as an effective mucosal healing agent with the potential to address an unmet need for the treatment of inflammatory

disorders of the mucosa. As current best medical treatment therapies, such as Anti-TNF $\alpha$ agents, have limited efficacy for the induction of mucosal healing $[15,16]$ and pose severe adverse reactions due to systemic administration [209-211]. In contrast, oral administration of CTB-KDEL might cause few, if any, systemic adverse effects and can directly heal lesions/ulcers and blunt inflammation. Therefore, CTB-KDEL provides a first-in-class therapeutic candidate for IBD that can effectively treat intestinal damage and induce mucosal healing without suppressing systemic immune functions, a significant unmet need in current IBD treatment. To aid in the development of CTBKDEL as a therapeutic agent, further research that delineates the detailed mechanisms by which CTB-KDEL induces mucosal healing is warranted. 


\section{REFERENCES}

1. $\quad \mathrm{Ng}, \mathrm{S} . \mathrm{C}$. , et al., Worldwide incidence and prevalence of inflammatory bowel disease in the 21st century: a systematic review of population-based studies. The Lancet. 390(10114): p. 2769-2778.

2. Kaser, A., S. Zeissig, and R.S. Blumberg, Inflammatory bowel disease. Annu Rev Immunol, 2010. 28: p. 573-621.

3. Saleh, M. and G. Trinchieri, Innate immune mechanisms of colitis and colitisassociated colorectal cancer. Nat Rev Immunol, 2011.

4. Iizuka, M. and S. Konno, Wound healing of intestinal epithelial cells. World J Gastroenterol, 2011. 17(17): p. 2161-71.

5. Neurath, M.F., New targets for mucosal healing and therapy in inflammatory bowel diseases. Mucosal Immunol, 2014. 7(1): p. 6-19.

6. $\quad$ Ferrari, L., M.K. Krane, and A. Fichera, Inflammatory bowel disease surgery in the biologic era. World J Gastrointest Surg, 2016. 8(5): p. 363-70.

7. Pineton de Chambrun, G., et al., Clinical implications of mucosal healing for the management of IBD. Nat Rev Gastroenterol Hepatol, 2010. 7(1): p. 15-29.

8. Orlando, A., et al., Clinical implications of mucosal healing in the management of patients with inflammatory bowel disease. Dig Liver Dis, 2013. 45(12): p. 986-91.

9. Boal Carvalho, P. and J. Cotter, Mucosal Healing in Ulcerative Colitis: A Comprehensive Review. Drugs, 2017. 77(2): p. 159-173.

10. Ardizzone, S., et al., Mucosal healing predicts late outcomes after the first course of corticosteroids for newly diagnosed ulcerative colitis. Clin Gastroenterol Hepatol, 2011. 9(6): p. 483-489 e3.

11. Rutgeerts, P., et al., Infliximab for induction and maintenance therapy for ulcerative colitis. N Engl J Med, 2005. 353(23): p. 2462-76.

12. Froslie, K.F., et al., Mucosal healing in inflammatory bowel disease: results from a Norwegian population-based cohort. Gastroenterology, 2007. 133(2): p. 41222.

13. Pagnini, C., et al., "Mucosal healing" in ulcerative colitis: Between clinical evidence and market suggestion. World J Gastrointest Pathophysiol, 2014. 5(2): p. 54-62.

14. Park, S.C. and Y.T. Jeen, Current and emerging biologics for ulcerative colitis. Gut Liver, 2015. 9(1): p. 18-27.

15. Villanacci, V., et al., Histological healing in inflammatory bowel disease: a still unfulfilled promise. World J Gastroenterol, 2013. 19(7): p. 968-78. 
16. Vaughn, B.P., S. Shah, and A.S. Cheifetz, The role of mucosal healing in the treatment of patients with inflammatory bowel disease. Curr Treat Options Gastroenterol, 2014. 12(1): p. 103-17.

17. Reinisch, W., et al., 52-week efficacy of adalimumab in patients with moderately to severely active ulcerative colitis who failed corticosteroids and/or immunosuppressants. Inflamm Bowel Dis, 2013. 19(8): p. 1700-9.

18. Leoni, G., et al., Wound repair: role of immune-epithelial interactions. Mucosal Immunol, 2015. 8(5): p. 959-68.

19. Walsh, A.J., R.V. Bryant, and S.P. Travis, Current best practice for disease activity assessment in IBD. Nat Rev Gastroenterol Hepatol, 2016. 13(10): p. 56779.

20. Finnson, K.W., et al., Dynamics of Transforming Growth Factor Beta Signaling in Wound Healing and Scarring. Adv Wound Care (New Rochelle), 2013. 2(5): p. 195-214.

21. Pakyari, M., et al., Critical Role of Transforming Growth Factor Beta in Different Phases of Wound Healing. Adv Wound Care (New Rochelle), 2013. 2(5): p. 215224.

22. Biancheri, P., et al., The role of transforming growth factor (TGF)-beta in modulating the immune response and fibrogenesis in the gut. Cytokine Growth Factor Rev, 2014. 25(1): p. 45-55.

23. Morikawa, M., R. Derynck, and K. Miyazono, TGF-beta and the TGF-beta Family: Context-Dependent Roles in Cell and Tissue Physiology. Cold Spring Harb Perspect Biol, 2016. 8(5).

24. Grivennikov, S.I., Inflammation and colorectal cancer: colitis-associated neoplasia. Semin Immunopathol, 2013. 35(2): p. 229-44.

25. Yashiro, M., Ulcerative colitis-associated colorectal cancer. World J Gastroenterol, 2014. 20(44): p. 16389-97.

26. Boirivant, M., et al., Inhibition of Smad7 with a specific antisense oligonucleotide facilitates TGF-betal-mediated suppression of colitis. Gastroenterology, 2006. 131(6): p. 1786-98.

27. Prevention, C.f.D.C.a., Cholera - Vibrio cholerae infection. 2018, U.S. Department of Health and Human Services.

28. Sanchez, J. and J. Holmgren, Cholera toxin structure, gene regulation and pathophysiological and immunological aspects. Cell Mol Life Sci, 2008. 65(9): p. 1347-60.

29. Finkelstein, R.A. and J.J. LoSpalluto, Pathogenesis of experimental cholera. Preparation and isolation of choleragen and choleragenoid. J Exp Med, 1969. 130(1): p. 185-202.

30. Lonnroth, I. and J. Holmgren, Subunit structure of cholera toxin. J Gen Microbiol, 1973. 76(2): p. 417-27.

31. Baldauf, K.J., et al., Cholera toxin B: one subunit with many pharmaceutical applications. Toxins (Basel), 2015. 7(3): p. 974-96. 
32. Cholera vaccines: WHO position paper. Wkly Epidemiol Rec, 2010. 85(13): p. 117-28.

33. Bergquist, C., et al., Intranasal vaccination of humans with recombinant cholera toxin B subunit induces systemic and local antibody responses in the upper respiratory tract and the vagina. Infect Immun, 1997. 65(7): p. 2676-84.

34. Jertborn, M., et al., Local and systemic immune responses to rectal administration of recombinant cholera toxin B subunit in humans. Infect Immun, 2001. 69(6): p. 4125-8.

35. Kozlowski, P.A., et al., Comparison of the oral, rectal, and vaginal immunization routes for induction of antibodies in rectal and genital tract secretions of women. Infect Immun, 1997. 65(4): p. 1387-94.

36. Cuatrecasas, P., Interaction of Vibrio cholerae enterotoxin with cell membranes. Biochemistry, 1973. 12(18): p. 3547-58.

37. Kuziemko, G.M., M. Stroh, and R.C. Stevens, Cholera toxin binding affinity and specificity for gangliosides determined by surface plasmon resonance.

Biochemistry, 1996. 35(20): p. 6375-84.

38. MacKenzie, C.R., et al., Quantitative analysis of bacterial toxin affinity and specificity for glycolipid receptors by surface plasmon resonance. J Biol Chem, 1997. 272(9): p. 5533-8.

39. Dawson, R.M., Characterization of the binding of cholera toxin to ganglioside GM1 immobilized onto microtitre plates. J Appl Toxicol, 2005. 25(1): p. 30-8.

40. George-Chandy, A., et al., Cholera toxin B subunit as a carrier molecule promotes antigen presentation and increases CD40 and CD86 expression on antigen-presenting cells. Infect Immun, 2001. 69(9): p. 5716-25.

41. Hamorsky, K.T., et al., Rapid and scalable plant-based production of a cholera toxin B subunit variant to aid in mass vaccination against cholera outbreaks. PLoS Negl Trop Dis, 2013. 7(3): p. e2046.

42. Hamorsky, K.T., et al., $N$-glycosylation of cholera toxin B subunit in Nicotiana benthamiana: impacts on host stress response, production yield and vaccine potential. Sci Rep, 2015. 5: p. 8003.

43. Matoba, N., N-Glycosylation of Cholera Toxin B Subunit: Serendipity for Novel Plant-Made Vaccines? Front Plant Sci, 2015. 6: p. 1132.

44. Moore, L., K. Hamorsky, and N. Matoba, Production of Recombinant Cholera Toxin B Subunit in Nicotiana benthamiana Using GENEWARE(R) Tobacco Mosaic Virus Vector. Methods Mol Biol, 2016. 1385: p. 129-37.

45. Sun, J.B., et al., Treatment of experimental autoimmune encephalomyelitis by feeding myelin basic protein conjugated to cholera toxin B subunit. Proc Natl Acad Sci U S A, 1996. 93(14): p. 7196-201.

46. Ploix, C., et al., Oral administration of cholera toxin B-insulin conjugates protects NOD mice from autoimmune diabetes by inducing $C D 4+$ regulatory $T$ cells. Diabetes, 1999. 48(11): p. 2150-6.

47. Tarkowski, A., et al., Treatment of experimental autoimmune arthritis by nasal administration of a type II collagen-cholera toxoid conjugate vaccine. Arthritis Rheum, 1999. 42(8): p. 1628-34.

48. Rask, C., et al., Prolonged oral treatment with low doses of allergen conjugated to cholera toxin B subunit suppresses immunoglobulin E antibody responses in sensitized mice. Clin Exp Allergy, 2000. 30(7): p. 1024-32. 
49. Stanford, M., et al., Oral tolerization with peptide 336-351 linked to cholera toxin $B$ subunit in preventing relapses of uveitis in Behcet's disease. Clin Exp Immunol, 2004. 137(1): p. 201-8.

50. Baldauf, K.J., et al., Oral administration of a recombinant cholera toxin B subunit promotes mucosal healing in the colon. Mucosal Immunol, 2017. 10(4): p. 887900.

51. Wernick, N.L., et al., Cholera toxin: an intracellular journey into the cytosol by way of the endoplasmic reticulum. Toxins (Basel), 2010. 2(3): p. 310-25.

52. Chinnapen, D.J., et al., Rafting with cholera toxin: endocytosis and trafficking from plasma membrane to ER. FEMS Microbiol Lett, 2007. 266(2): p. 129-37.

53. Zhang, R.G., et al., The three-dimensional crystal structure of cholera toxin. $\mathrm{J}$ Mol Biol, 1995. 251(4): p. 563-73.

54. Lencer, W.I., et al., Targeting of cholera toxin and Escherichia coli heat labile toxin in polarized epithelia: role of COOH-terminal KDEL. J Cell Biol, 1995. 131(4): p. 951-62.

55. Fujinaga, Y., et al., Gangliosides that associate with lipid rafts mediate transport of cholera and related toxins from the plasma membrane to endoplasmic reticulm. Mol Biol Cell, 2003. 14(12): p. 4783-93.

56. Sanchez, J. and J. Holmgren, Cholera toxin - a foe \& a friend. Indian J Med Res, 2011. 133: p. 153-63.

57. Sun, J.B., J. Holmgren, and C. Czerkinsky, Cholera toxin B subunit: an efficient transmucosal carrier-delivery system for induction of peripheral immunological tolerance. Proc Natl Acad Sci U S A, 1994. 91(23): p. 10795-9.

58. Weiner, H.L., Oral tolerance. Proc Natl Acad Sci U S A, 1994. 91(23): p. 107625.

59. Bublin, M., et al., Use of a genetic cholera toxin B subunit/allergen fusion molecule as mucosal delivery system with immunosuppressive activity against Th2 immune responses. Vaccine, 2007. 25(50): p. 8395-404.

60. Ruhlman, T., et al., Expression of cholera toxin B-proinsulin fusion protein in lettuce and tobacco chloroplasts--oral administration protects against development of insulitis in non-obese diabetic mice. Plant Biotechnol J, 2007. 5(4): p. 495-510.

61. Carter, J.E., 3rd, et al., Bacterial and plant enterotoxin B subunit-autoantigen fusion proteins suppress diabetes insulitis. Mol Biotechnol, 2006. 32(1): p. 1-15.

62. Arakawa, T., et al., A plant-based cholera toxin B subunit-insulin fusion protein protects against the development of autoimmune diabetes. Nat Biotechnol, 1998. 16(10): p. 934-8.

63. Sun, J.B., et al., Intranasal administration of a Schistosoma mansoni glutathione $S$-transferase-cholera toxoid conjugate vaccine evokes antiparasitic and antipathological immunity in mice. J Immunol, 1999. 163(2): p. 1045-52.

64. McSorley, S.J., et al., Selective tolerization of Th1-like cells after nasal administration of a cholera toxoid-LACK conjugate. Eur J Immunol, 1998. 28(2): p. 424-32.

65. Czerkinsky, C., et al., Mucosal immunity and tolerance: relevance to vaccine development. Immunol Rev, 1999. 170: p. 197-222. 
66. Phipps, P.A., et al., Prevention of mucosally induced uveitis with a HSP60derived peptide linked to cholera toxin B subunit. Eur J Immunol, 2003. 33(1): p. 224-32.

67. Sun, J.B., et al., Oral administration of cholera toxin B subunit conjugated to myelin basic protein protects against experimental autoimmune encephalomyelitis by inducing transforming growth factor-beta-secreting cells and suppressing chemokine expression. Int Immunol, 2000. 12(10): p. 1449-57.

68. Bergerot, I., et al., A cholera toxoid-insulin conjugate as an oral vaccine against spontaneous autoimmune diabetes. Proc Natl Acad Sci U S A, 1997. 94(9): p. 4610-4.

69. Sun, J.B., C. Czerkinsky, and J. Holmgren, Mucosally induced immunological tolerance, regulatory $T$ cells and the adjuvant effect by cholera toxin B subunit. Scand J Immunol, 2010. 71(1): p. 1-11.

70. Boirivant, M., et al., Oral administration of recombinant cholera toxin subunit B inhibits IL-12-mediated murine experimental (trinitrobenzene sulfonic acid) colitis. J Immunol, 2001. 166(5): p. 3522-32.

71. Tamura, S., et al., Synergistic action of cholera toxin B subunit (and Escherichia coli heat-labile toxin B subunit) and a trace amount of cholera whole toxin as an adjuvant for nasal influenza vaccine. Vaccine, 1994. 12(5): p. 419-26.

72. Kim, P.H., et al., Cholera toxin and cholera toxin B subunit induce IgA switching through the action of TGF-beta 1. J Immunol, 1998. 160(3): p. 1198-203.

73. Reinholdt J, H.S., IgA and Mucosal Homeostasis. Madame Curie Bioscience Database, 2000-2013.

74. Corthesy, B., Role of secretory IgA in infection and maintenance of homeostasis. Autoimmunity reviews, 2013. 12(6): p. 661-5.

75. Smits, H.H., et al., Cholera toxin B suppresses allergic inflammation through induction of secretory IgA. Mucosal Immunol, 2009. 2(4): p. 331-9.

76. Neurath, M.F., S. Finotto, and L.H. Glimcher, The role of Th1/Th2 polarization in mucosal immunity. Nat Med, 2002. 8(6): p. 567-73.

77. Coccia, E.M., et al., Cholera toxin subunit B inhibits IL-12 and IFN-\{gamma\} production and signaling in experimental colitis and Crohn's disease. Gut, 2005. 54(11): p. 1558-64.

78. Stal, P., et al., Clinical trial: the safety and short-term efficacy of recombinant cholera toxin B subunit in the treatment of active Crohn's disease. Aliment Pharmacol Ther, 2010. 31(3): p. 387-95.

79. Aman, A.T., et al., A mutant cholera toxin B subunit that binds GM1-ganglioside but lacks immunomodulatory or toxic activity. Proc Natl Acad Sci U S A, 2001. 98(15): p. 8536-41.

80. D'Ambrosio, A., et al., Cholera toxin B subunit promotes the induction of regulatory $T$ cells by preventing human dendritic cell maturation. J Leukoc Biol, 2008. 84(3): p. 661-8.

81. Rouquette-Jazdanian, A.K., et al., Cholera toxin B-subunit prevents activation and proliferation of human CD4+ T cells by activation of a neutral sphingomyelinase in lipid rafts. J Immunol, 2005. 175(9): p. 5637-48.

82. Dbaibo, G.S., et al., Retinoblastoma gene product as a downstream target for a ceramide-dependent pathway of growth arrest. Proc Natl Acad Sci U S A, 1995. 92(5): p. 1347-51. 
83. Lee, J.Y., Y.A. Hannun, and L.M. Obeid, Ceramide inactivates cellular protein kinase Calpha. J Biol Chem, 1996. 271(22): p. 13169-74.

84. Lee, J.Y., Y.A. Hannun, and L.M. Obeid, Functional dichotomy of protein kinase $C(P K C)$ in tumor necrosis factor-alpha (TNF-alpha) signal transduction in L929 cells. Translocation and inactivation of PKC by TNF-alpha. J Biol Chem, 2000. 275(38): p. 29290-8.

85. Leoni, G., et al., Wound repair: role of immune-epithelial interactions. Mucosal immunology, 2015. 8(5): p. 959-68.

86. Kurashima, Y. and H. Kiyono, Mucosal Ecological Network of Epithelium and Immune Cells for Gut Homeostasis and Tissue Healing. Annu Rev Immunol, 2017. 35: p. 119-147.

87. Ma, D., et al., Interleukin-10 and nerve growth factor have reciprocal upregulatory effects on intestinal epithelial cells. Am J Physiol Regul Integr Comp Physiol, 2003. 284(5): p. R1323-9.

88. Stordeur, P. and M. Goldman, Interleukin-10 as a regulatory cytokine induced by cellular stress: molecular aspects. Int Rev Immunol, 1998. 16(5-6): p. 501-22.

89. Hamorsky, K.T., et al., Rapid and scalable plant-based production of a cholera toxin $B$ subunit variant to aid in mass vaccination against cholera outbreaks. PLoS neglected tropical diseases, 2013. 7(3): p. e2046.

90. Zhang, L., et al., Anti-inflammatory effect of cholera toxin B subunit in experimental stroke. J Neuroinflammation, 2016. 13(1): p. 147.

91. Aspord, C., et al., alpha4 integrins and L-selectin differently orchestrate T-cell activity during diabetes prevention following oral administration of CTB-insulin. J Autoimmun, 2002. 19(4): p. 223-32.

92. Wynn, T.A. and K.M. Vannella, Macrophages in Tissue Repair, Regeneration, and Fibrosis. Immunity, 2016. 44(3): p. 450-462.

93. Satoh, Y., et al., Cyclosporine regulates intestinal epithelial apoptosis via TGFbeta-related signaling. Am J Physiol Gastrointest Liver Physiol, 2009. 297(3): p. G514-9.

94. Taverna, D., et al., Imaging mass spectrometry for assessing cutaneous wound healing: analysis of pressure ulcers. J Proteome Res, 2015. 14(2): p. 986-96.

95. Kiesler, P., I.J. Fuss, and W. Strober, Experimental Models of Inflammatory Bowel Diseases. Cell Mol Gastroenterol Hepatol, 2015. 1(2): p. 154-170.

96. Perse, M. and A. Cerar, Dextran sodium sulphate colitis mouse model: traps and tricks. J Biomed Biotechnol, 2012. 2012: p. 718617.

97. Clapper, M.L., H.S. Cooper, and W.C. Chang, Dextran sulfate sodium-induced colitis-associated neoplasia: a promising model for the development of chemopreventive interventions. Acta Pharmacol Sin, 2007. 28(9): p. 1450-9.

98. Jiminez, J.A., et al., Animal models to study acute and chronic intestinal inflammation in mammals. Gut Pathog, 2015. 7: p. 29.

99. Chassaing, B., et al., Dextran sulfate sodium (DSS)-induced colitis in mice. Curr Protoc Immunol, 2014. 104: p. Unit 15.25.

100. Terzic, J., et al., Inflammation and colon cancer. Gastroenterology, 2010. 138(6): p. 2101-2114.e5.

101. Jawad, N., N. Direkze, and S.J. Leedham, Inflammatory bowel disease and colon cancer. Recent Results Cancer Res, 2011. 185: p. 99-115. 
102. Denecke, J., R. De Rycke, and J. Botterman, Plant and mammalian sorting signals for protein retention in the endoplasmic reticulum contain a conserved epitope. Embo j, 1992. 11(6): p. 2345-55.

103. Hamorsky, K. and N. Matoba, Facile Method for the Production of Recombinant Cholera Toxin B Subunit in E. coli. Methods Mol Biol, 2016. 1404: p. 511-8.

104. Dionne, S., et al., Colonic explant production of IL-1and its receptor antagonist is imbalanced in inflammatory bowel disease (IBD). Clin Exp Immunol, 1998. 112(3): p. 435-42.

105. Randall, K.J., J. Turton, and J.R. Foster, Explant culture of gastrointestinal tissue: a review of methods and applications. Cell Biol Toxicol, 2011. 27(4): p. 267-84.

106. Clemens, J., et al., New-generation vaccines against cholera. Nat Rev Gastroenterol Hepatol, 2011. 8(12): p. 701-10.

107. Royal, J.M. and N. Matoba, Therapeutic Potential of Cholera Toxin B Subunit for the Treatment of Inflammatory Diseases of the Mucosa. Toxins (Basel), 2017. 9(12).

108. Capitani, M. and M. Sallese, The KDEL receptor: new functions for an old protein. FEBS Lett, 2009. 583(23): p. 3863-71.

109. Cabrera, M., et al., The retrieval function of the KDEL receptor requires PKA phosphorylation of its C-terminus. Mol Biol Cell, 2003. 14(10): p. 4114-25.

110. Becker, B., et al., Cargo binding promotes KDEL receptor clustering at the mammalian cell surface. Sci Rep, 2016. 6: p. 28940.

111. Bagchi, S., R. Fredriksson, and A. Wallen-Mackenzie, In Situ Proximity Ligation Assay (PLA). Methods Mol Biol, 2015. 1318: p. 149-59.

112. Lamriben, L., et al., N-Glycan-based ER Molecular Chaperone and Protein Quality Control System: The Calnexin Binding Cycle. Traffic, 2016. 17(4): p. 308-26.

113. Eletto, D., et al., Protein disulfide isomerase A6 controls the decay of IRElalpha signaling via disulfide-dependent association. Mol Cell, 2014. 53(4): p. 562-576.

114. Sepulveda, D., et al., Interactome Screening Identifies the ER Luminal Chaperone Hsp47 as a Regulator of the Unfolded Protein Response Transducer IRElalpha. Mol Cell, 2018. 69(2): p. 238-252.e7.

115. Cho, J.A., et al., The unfolded protein response element IRElalpha senses bacterial proteins invading the ER to activate RIG-I and innate immune signaling. Cell Host Microbe, 2013. 13(5): p. 558-69.

116. Walter, P. and D. Ron, The unfolded protein response: from stress pathway to homeostatic regulation. Science, 2011. 334(6059): p. 1081-6.

117. Ron, D. and P. Walter, Signal integration in the endoplasmic reticulum unfolded protein response. Nat Rev Mol Cell Biol, 2007. 8(7): p. 519-29.

118. Bertolotti, A., et al., Increased sensitivity to dextran sodium sulfate colitis in IRE1beta-deficient mice. J Clin Invest, 2001. 107(5): p. 585-93.

119. Zhang, H., et al., Colitis Is Effectively Ameliorated by (+/-)-8-Acetonyldihydrocoptisine via the XBP1-NF-kappaB Pathway. Front Pharmacol, 2017. 8: p. 619.

120. Wang, J.M., et al., Inositol-Requiring Enzyme 1 Facilitates Diabetic Wound Healing Through Modulating MicroRNAs. Diabetes, 2017. 66(1): p. 177-192.

121. McCaffrey, K. and I. Braakman, Protein quality control at the endoplasmic reticulum. Essays Biochem, 2016. 60(2): p. 227-235. 
122. Frakes, A.E. and A. Dillin, The UPR(ER): Sensor and Coordinator of Organismal Homeostasis. Mol Cell, 2017. 66(6): p. 761-771.

123. Zhang, L., et al., IRE1 inhibition perturbs the unfolded protein response in a pancreatic beta-cell line expressing mutant proinsulin, but does not sensitize the cells to apoptosis. BMC Cell Biol, 2014. 15: p. 29.

124. Dame, M.K., et al., Human colonic crypts in culture: segregation of immunochemical markers in normal versus adenoma-derived. Lab Invest, 2014. 94(2): p. 222-34.

125. Zeisberg, M. and E.G. Neilson, Biomarkers for epithelial-mesenchymal transitions. J Clin Invest, 2009. 119(6): p. 1429-37.

126. Luissint, A.C., C.A. Parkos, and A. Nusrat, Inflammation and the Intestinal Barrier: Leukocyte-Epithelial Cell Interactions, Cell Junction Remodeling, and Mucosal Repair. Gastroenterology, 2016. 151(4): p. 616-32.

127. Zhang, D.L., et al., Effect of Wnt signaling pathway on wound healing. Biochem Biophys Res Commun, 2009. 378(2): p. 149-51.

128. Sturm, A. and A.U. Dignass, Epithelial restitution and wound healing in inflammatory bowel disease. World J Gastroenterol, 2008. 14(3): p. 348-53.

129. Park, H.J., et al., Electrical Stimulation Modulates the Expression of Multiple Wound Healing Genes in Primary Human Dermal Fibroblasts. Tissue Eng Part A, 2015. 21(13-14): p. 1982-90.

130. Yamamoto, K., et al., The KDEL receptor mediates a retrieval mechanism that contributes to quality control at the endoplasmic reticulum. Embo j, 2001. 20(12): p. 3082-91.

131. Yamamoto, K., et al., The KDEL receptor modulates the endoplasmic reticulum stress response through mitogen-activated protein kinase signaling cascades. $\mathbf{J}$ Biol Chem, 2003. 278(36): p. 34525-32.

132. Chusri, P., et al., HCV induces transforming growth factor betal through activation of endoplasmic reticulum stress and the unfolded protein response. Sci Rep, 2016. 6: p. 22487.

133. Matsuzaki, S., et al., Physiological ER Stress Mediates the Differentiation of Fibroblasts. PLoS One, 2015. 10(4): p. e0123578.

134. Roberson, E.C., et al., Influenza induces endoplasmic reticulum stress, caspase12-dependent apoptosis, and c-Jun N-terminal kinase-mediated transforming growth factor-beta release in lung epithelial cells. Am J Respir Cell Mol Biol, 2012. 46(5): p. 573-81.

135. Geiger, R., et al., Investigating endocytic pathways to the endoplasmic reticulum and to the cytosol using SNAP-trap. Traffic, 2013. 14(1): p. 36-46.

136. Todd, D.J., A.H. Lee, and L.H. Glimcher, The endoplasmic reticulum stress response in immunity and autoimmunity. Nat Rev Immunol, 2008. 8(9): p. 66374.

137. Schroder, M. and R.J. Kaufman, The mammalian unfolded protein response. Annu Rev Biochem, 2005. 74: p. 739-89.

138. Kaser, A., et al., The unfolded protein response and its role in intestinal homeostasis and inflammation. Exp Cell Res, 2011. 317(19): p. 2772-9.

139. Cao, S.S., B. Song, and R.J. Kaufman, PKR protects colonic epithelium against colitis through the unfolded protein response and prosurvival signaling. Inflamm Bowel Dis, 2012. 18(9): p. 1735-42. 
140. Lacy, E.R. and S. Ito, Rapid epithelial restitution of the rat gastric mucosa after ethanol injury. Lab Invest, 1984. 51(5): p. 573-83.

141. Dignass, A.U. and D.K. Podolsky, Cytokine modulation of intestinal epithelial cell restitution: central role of transforming growth factor beta. Gastroenterology, 1993. 105(5): p. 1323-32.

142. Cho, H.J., et al., Snail is required for transforming growth factor-beta-induced epithelial-mesenchymal transition by activating PI3 kinase/Akt signal pathway. Biochem Biophys Res Commun, 2007. 353(2): p. 337-43.

143. Leight, J.L., et al., Matrix rigidity regulates a switch between TGF-betal-induced apoptosis and epithelial-mesenchymal transition. Mol Biol Cell, 2012. 23(5): p. 781-91.

144. Miyoshi, H., et al., Wnt5a potentiates TGF-beta signaling to promote colonic crypt regeneration after tissue injury. Science, 2012. 338(6103): p. 108-13.

145. Hwang, S., et al., E-cadherin is critical for collective sheet migration and is regulated by the chemokine CXCL12 protein during restitution. J Biol Chem, 2012. 287(26): p. 22227-40.

146. Araki, K., et al., E/N-cadherin switch mediates cancer progression via TGF-betainduced epithelial-to-mesenchymal transition in extrahepatic cholangiocarcinoma. Br J Cancer, 2011. 105(12): p. 1885-93.

147. Royal, J.M., et al., A modified cholera toxin B subunit containing an ER retention motif enhances colon epithelial repair via an unfolded protein response. Faseb j, 2019: p. fj201901255R.

148. Dahlhamer, J.M., et al., Prevalence of Inflammatory Bowel Disease Among Adults Aged >/=18 Years - United States, 2015. MMWR Morb Mortal Wkly Rep, 2016. 65(42): p. 1166-1169.

149. Wirtz, S., et al., Chemically induced mouse models of acute and chronic intestinal inflammation. Nat Protoc, 2017. 12(7): p. 1295-1309.

150. Das, S., S.K.K. Batra, and S. Rachagani, Mouse Model of Dextran Sodium Sulfate (DSS)-induced Colitis. Bio-protocol, 2017. 7(16): p. e2515.

151. Pasetti, M.F., et al., Immunology of gut mucosal vaccines. Immunological reviews, 2011. 239(1): p. 125-148.

152. Yin, L., et al., Therapeutic outcomes, assessments, risk factors and mitigation efforts of immunogenicity of therapeutic protein products. Cell Immunol, 2015. 295(2): p. 118-26.

153. Swanson, S.J. and J. Bussiere, Immunogenicity assessment in non-clinical studies. Curr Opin Microbiol, 2012. 15(3): p. 337-47.

154. Cooper, H.S., et al., Clinicopathologic study of dextran sulfate sodium experimental murine colitis. Lab Invest, 1993. 69(2): p. 238-49.

155. Eichele, D.D. and K.K. Kharbanda, Dextran sodium sulfate colitis murine model: An indispensable tool for advancing our understanding of inflammatory bowel diseases pathogenesis. World J Gastroenterol, 2017. 23(33): p. 6016-6029.

156. Griseri, T., et al., Dysregulated hematopoietic stem and progenitor cell activity promotes interleukin-23-driven chronic intestinal inflammation. Immunity, 2012. 37(6): p. 1116-29.

157. Cong, Y., H.R. Bowdon, and C.O. Elson, Identification of an immunodominant T cell epitope on cholera toxin. European Journal of Immunology, 1996. 26(11): p. 2587-2594. 
158. Takahashi, I., et al., Mechanisms for mucosal immunogenicity and adjuvancy of Escherichia coli labile enterotoxin. J Infect Dis, 1996. 173(3): p. 627-35.

159. Mombaerts, P., et al., RAG-1-deficient mice have no mature B and T lymphocytes. Cell, 1992. 68(5): p. 869-77.

160. DeVoss, J. and L. Diehl, Murine models of inflammatory bowel disease (IBD): challenges of modeling human disease. Toxicol Pathol, 2014. 42(1): p. 99-110.

161. Soderholm, J.D., et al., Increased epithelial uptake of protein antigens in the ileum of Crohn's disease mediated by tumour necrosis factor alpha. Gut, 2004. 53(12): p. 1817-24.

162. Wang, F., et al., Interferon-gamma and tumor necrosis factor-alpha synergize to induce intestinal epithelial barrier dysfunction by up-regulating myosin light chain kinase expression. Am J Pathol, 2005. 166(2): p. 409-19.

163. Marini, M., et al., TNF-alpha neutralization ameliorates the severity of murine Crohn's-like ileitis by abrogation of intestinal epithelial cell apoptosis. Proc Natl Acad Sci U S A, 2003. 100(14): p. 8366-71.

164. Okamoto, R. and M. Watanabe, Cellular and molecular mechanisms of the epithelial repair in IBD. Dig Dis Sci, 2005. 50 Suppl 1: p. S34-8.

165. Gulwani, H. Ulcerative colitis. 2014; Available from: http://www.pathologyoutlines.com/topic/colonuc.html. .

166. Tursi, A., Histologic healing in inflammatory bowel disease clinical practice: a reliable target? Clin Gastroenterol Hepatol, 2015. 13(6): p. 1211-2.

167. Peyrin-Biroulet, L., A. Bressenot, and W. Kampman, Histologic remission: the ultimate therapeutic goal in ulcerative colitis? Clin Gastroenterol Hepatol, 2014. 12(6): p. 929-34.e2.

168. Yu, Y.R. and J.R. Rodriguez, Clinical presentation of Crohn's, ulcerative colitis, and indeterminate colitis: Symptoms, extraintestinal manifestations, and disease phenotypes. Semin Pediatr Surg, 2017. 26(6): p. 349-355.

169. Schellekens, H., The immunogenicity of therapeutic proteins. Discov Med, 2010. 9(49): p. 560-4.

170. Sauna, Z.E., et al., Evaluating and Mitigating the Immunogenicity of Therapeutic Proteins. Trends Biotechnol, 2018.

171. Brinks, V., W. Jiskoot, and H. Schellekens, Immunogenicity of therapeutic proteins: the use of animal models. Pharm Res, 2011. 28(10): p. 2379-85.

172. Gutzeit, C., G. Magri, and A. Cerutti, Intestinal IgA production and its role in host-microbe interaction. Immunol Rev, 2014. 260(1): p. 76-85.

173. Mathias, A., et al., Role of secretory IgA in the mucosal sensing of commensal bacteria. Gut Microbes, 2014. 5(6): p. 688-95.

174. Mkaddem, S.B., et al., IgA, IgA receptors, and their anti-inflammatory properties. Curr Top Microbiol Immunol, 2014. 382: p. 221-35.

175. Dominguez-Villar, M. and D.A. Hafler, Regulatory T cells in autoimmune disease. Nature Immunology, 2018. 19(7): p. 665-673.

176. Justice, M.J. and P. Dhillon, Using the mouse to model human disease: increasing validity and reproducibility. Dis Model Mech, 2016. 9(2): p. 101-3.

177. Tarnawski, A.S. and A. Ahluwalia, Increased susceptibility of aging gastric mucosa to injury and delayed healing: Clinical implications. World Journal of Gastroenterology, 2018. 24(42): p. 4721-4727. 
178. Okamoto, R. and M. Watanabe, Role of epithelial cells in the pathogenesis and treatment of inflammatory bowel disease. J Gastroenterol, 2016. 51(1): p. 11-21.

179. Merritt, E.A., et al., Crystal structure of cholera toxin B-pentamer bound to receptor GM1 pentasaccharide. Protein Sci, 1994. 3(2): p. 166-75.

180. Yanagisawa, M., T. Ariga, and R.K. Yu, Cholera toxin B subunit binding does not correlate with GM1 expression: a study using mouse embryonic neural precursor cells. Glycobiology, 2006. 16(9): p. 19g-22g.

181. Lauer, S., et al., Analysis of cholera toxin-ganglioside interactions by flow cytometry. Biochemistry, 2002. 41(6): p. 1742-51.

182. Blank, N., et al., Cholera toxin binds to lipid rafts but has a limited specificity for ganglioside GM1. Immunol Cell Biol, 2007. 85(5): p. 378-82.

183. Tessitore, A., et al., GM1-ganglioside-mediated activation of the unfolded protein response causes neuronal death in a neurodegenerative gangliosidosis. Mol Cell, 2004. 15(5): p. 753-66.

184. Pelled, D., et al., Inhibition of calcium uptake via the sarco/endoplasmic reticulum Ca2+-ATPase in a mouse model of Sandhoff disease and prevention by treatment with N-butyldeoxynojirimycin. J Biol Chem, 2003. 278(32): p. 29496501.

185. Sano, R., et al., GM1-ganglioside accumulation at the mitochondria-associated ER membranes links ER stress to Ca(2+)-dependent mitochondrial apoptosis. Mol Cell, 2009. 36(3): p. 500-11.

186. Pincus, D., et al., BiP binding to the ER-stress sensor Ire1 tunes the homeostatic behavior of the unfolded protein response. PLoS Biol, 2010. 8(7): p. e1000415.

187. Credle, J.J., et al., On the mechanism of sensing unfolded protein in the endoplasmic reticulum. Proc Natl Acad Sci U S A, 2005. 102(52): p. 18773-84.

188. Knorr, R., C. Karacsonyi, and R. Lindner, Endocytosis of MHC molecules by distinct membrane rafts. J Cell Sci, 2009. 122(Pt 10): p. 1584-94.

189. Zhou, J., et al., The crystal structure of human IRE1 luminal domain reveals a conserved dimerization interface required for activation of the unfolded protein response. Proc Natl Acad Sci U S A, 2006. 103(39): p. 14343-8.

190. Gong, J., et al., Molecular signal networks and regulating mechanisms of the unfolded protein response. J Zhejiang Univ Sci B, 2017. 18(1): p. 1-14.

191. Frakes, A.E. and A. Dillin, The UPRER: Sensor and Coordinator of Organismal Homeostasis. Mol Cell, 2017. 66(6): p. 761-771.

192. Jin, H., M. Komita, and T. Aoe, The Role of BiP Retrieval by the KDEL Receptor in the Early Secretory Pathway and its Effect on Protein Quality Control and Neurodegeneration. Front Mol Neurosci, 2017. 10: p. 222.

193. Laping, N.J., et al., Inhibition of transforming growth factor (TGF)-betal-induced extracellular matrix with a novel inhibitor of the TGF-beta type I receptor kinase activity: SB-431542. Mol Pharmacol, 2002. 62(1): p. 58-64.

194. Moynagh, P.N., The NF- $\kappa B$ pathway. Journal of Cell Science, 2005. 118(20): p. 4589-4592.

195. Karin, M., et al., NF-kappaB in cancer: from innocent bystander to major culprit. Nat Rev Cancer, 2002. 2(4): p. 301-10.

196. Park, M.H. and J.T. Hong, Roles of NF-kappaB in Cancer and Inflammatory Diseases and Their Therapeutic Approaches. Cells, 2016. 5(2). 
197. Biancheri, P., et al., The role of transforming growth factor (TGF)-beta in modulating the immune response and fibrogenesis in the gut. Cytokine \& growth factor reviews, 2014. 25(1): p. 45-55.

198. Travis, M.A. and D. Sheppard, TGF-beta activation and function in immunity. Annual review of immunology, 2014. 32: p. 51-82.

199. Penn, J.W., A.O. Grobbelaar, and K.J. Rolfe, The role of the TGF-beta family in wound healing, burns and scarring: a review. Int J Burns Trauma, 2012. 2(1): p. 18-28.

200. Kim, M.K., et al., The differential expression of TGF-betal, ILK and wnt signaling inducing epithelial to mesenchymal transition in human renal fibrogenesis: an immunohistochemical study. Int J Clin Exp Pathol, 2013. 6(9): p. 1747-58.

201. Zhang, Y.Q., et al., Resveratrol ameliorates lipopolysaccharide-induced epithelial mesenchymal transition and pulmonary fibrosis through suppression of oxidative stress and transforming growth factor-betal signaling. Clin Nutr, 2015. 34(4): p. 752-60.

202. Colak, S. and P. Ten Dijke, Targeting TGF-beta Signaling in Cancer. Trends Cancer, 2017. 3(1): p. 56-71.

203. Crespo, M., et al., Colonic organoids derived from human induced pluripotent stem cells for modeling colorectal cancer and drug testing. Nat Med, 2017. 23(7): p. 878-884.

204. Laukoetter, M.G., et al., JAM-A regulates permeability and inflammation in the intestine in vivo. J Exp Med, 2007. 204(13): p. 3067-76.

205. Kong, J., et al., Novel role of the vitamin D receptor in maintaining the integrity of the intestinal mucosal barrier. Am J Physiol Gastrointest Liver Physiol, 2008. 294(1): p. G208-16.

206. Chogle, A., et al., Milk fat globule-EGF factor 8 is a critical protein for healing of dextran sodium sulfate-induced acute colitis in mice. Mol Med, 2011. 17(5-6): p. 502-7.

207. Ukena, S.N., et al., Probiotic Escherichia coli Nissle 1917 inhibits leaky gut by enhancing mucosal integrity. PLoS One, 2007. 2(12): p. e1308.

208. Neurath, M.F., et al., Experimental granulomatous colitis in mice is abrogated by induction of TGF-beta-mediated oral tolerance. J Exp Med, 1996. 183(6): p. 2605-16.

209. Hansel, T.T., et al., The safety and side effects of monoclonal antibodies. Nat Rev Drug Discov, 2010. 9(4): p. 325-38.

210. Murdaca, G., et al., Infection risk associated with anti-TNF-alpha agents: a review. Expert Opin Drug Saf, 2015. 14(4): p. 571-82.

211. Saleh, M. and G. Trinchieri, Innate immune mechanisms of colitis and colitisassociated colorectal cancer. Nat Rev Immunol, 2011. 11(1): p. 9-20. 


\title{
CURRICULUM VITAE
}

\author{
Joshua Royal \\ Clinical and Translational Research Building, Lab 626b \\ 505 S. Hancock Street \\ Louisville, KY 40202 \\ (270) 315-4205 \\ E-mail: Joshua.royal@louisville.edu
}

\section{EDUCATION}

2009-2013

B.S. in Molecular Biology with a minor in Psychology, Western Kentucky University, Ogden College of Science and Engineering, Bowling Green, KY

2016-2018 M.S. in Pharmacology and Toxicology, Department of Pharmacology and Toxicology, University of Louisville School of Medicine, Louisville, KY (Advisor: Nobuyuki Matoba, Ph.D.)

2018-TBD Ph.D. in Pharmacology and Toxicology, Department of Pharmacology and Toxicology, University of Louisville School of Medicine, Louisville, KY (Advisor: Nobuyuki Matoba, Ph.D.)

\section{INDUSTRY EMPLOYMENT}

1/2019 - present Clinical Regulatory Affairs Specialist

US WorldMeds, Louisville, KY

\section{ACADEMIC APPOINTMENTS}

\author{
1/2017 - $2018 \quad$ Research Technologist II/Research Lab Manager \\ James Graham Brown Cancer Center, University of Louisville School of \\ Medicine, Louisville, KY \\ 5/2014-1/2017 Research Technician \\ James Graham Brown Cancer Center, University of Louisville School of \\ Medicine, Louisville, KY
}




\title{
OTHER POSITIONS AND EMPLOYMENT
}

\author{
2/2017 - $2018 \quad$ PREVENT GLP Lab Manager \\ Biopharmaceutical Research Unit, University of Louisville, Louisville, \\ KY \\ 9/2013 - 4/2014 Research Technician \\ Owensboro Cancer Research Program, Owensboro Health, Owensboro, \\ $\mathrm{KY}$
}

\section{PROFESSIONAL MEMBERSHIPS AND ACTIVITIES}

$3 / 2018$ - present

9/2017 - Present

6/2013 - Present
Member of the American Gastroenterology Association

Member of the American Association for the Advancement of Science

Member of the National Animal Health Emergency Response Corps

\section{HONORS AND AWARDS}

2014

2014

2016

2016

2017

2018

2018

2018

2018

2018

2018

2019
Research Staff Award (1st Place), Research Louisville 2014, University of Louisville.

Roving Research Award ( $\mathbf{2}^{\text {nd }}$ Place), James Graham Brown Cancer Center $14^{\text {th }}$ Annual Retreat.

Masters Basic-Science Graduate Student Award ( $2^{\text {nd }}$ Place $)$, Research Louisville 2016, University of Louisville.

Best Presentation Award ( $\mathbf{1}^{\text {st }}$ Place), Center for Predictive Medicine Retreat 2016.

Masters Basic-Science Graduate Student Award ( $1^{\text {st }}$ Place), Research Louisville 2017, University of Louisville.

Graduate Merit Scholarship, University of Louisville Graduate School Council.

Basic Science Travel Award, Digestive Disease Week 2018

Certificate of Recognition for Scientific Accomplishment as an Early

Career Investigator, American Gastroenterology Association

Certificate of Recognition for Early Career Stage Basic Science

Investigator, Digestive Disease Week 2018

Doctoral Basic-Science Graduate Student Award ( $1^{\text {st }}$ Place $)$,

Research Louisville 2018, University of Louisville.

Graduate Merit Scholarship, School of Interdisciplinary and Graduate Studies, University of Louisville

Travel Award, Executive Vice President for Research and Innovation, University of Louisville 


$\begin{array}{ll}\text { 9/2013 - 9/2019 } & \text { NIH Guidelines } \\ 6 / 2014-6 / 2019 & \text { Basic Biosafety Training } \\ 6 / 2014 & \text { HIPAA Privacy Training (Non-Research) } \\ 6 / 2014 & \text { HIPAA Security/Information Security } \\ 8 / 2015-8 / 2019 & \text { Laboratory Safety and Hazardous Waste Training } \\ \text { 9/2015 -9/2019 } & \text { Hazardous Waste Training } \\ 3 / 2016-3 / 2020 & \text { Human Subjects and HIPAA-Research/Biomedical Research } \\ 3 / 2016-2 / 2020 & \text { Institutional Compliance - U of L General Population } \\ 6 / 2017-6 / 2019 & \text { Bloodborne Pathogens Training } \\ 7 / 2017-7 / 2020 & \text { Occupational Health and Safety Training for Animal Handlers } \\ 4 / 2017-4 / 2019 & \text { Good Laboratory Practice Training: for Non-Clinical Testing, Standard } \\ & \text { Operation Procedures } \\ 4 / 2017-4 / 2019 & \text { GLP Training: SOP Implementation }\end{array}$

\section{EDUCATIONAL ACTIVITIES}

Mentoring/Training of undergraduate and high-school students

$\begin{array}{ll}\text { 6/2016 - 9/2016 } & \text { Ms. Nivedha Loganathan, Dupont Manual High School } \\ \text { 6/2017 - 12/2017 } & \text { Ms. Nivedha Loganathan, Dupont Manual High School } \\ \text { 5/2017 - present } & \text { Mr. David Morris, University of Louisville }\end{array}$

$\underline{\text { Lectures in invited seminars }}$

1. Royal J, Baldauf K, Yaddanapudi K, Dryden GW, Matoba N. “CTBp, a Plant-Made Oral Immunotherapeutic against Ulcerative Colitis" James Graham Brown Cancer Center Colloquia on Cancer Biology and Therapeutics, Louisville, KY, 2015

2. Royal J, Youngjun Oh, Matoba N. "Epithelial Cell ER Targeted Protein = Epicertin" James Graham Brown Cancer Center Colloquia on Cancer Biology and Therapeutics, Louisville, KY, 2017

3. Royal J, Matoba N. "Plant-Made Anti-TNF $\alpha$ Monoclonal Antibody as an Oral Biopharmaceutical Agent against Intestinal Inflammation and Colon Cancer" Owensboro Cancer Research Program. April 2014, Owensboro, KY

4. Royal J, Matoba N. "Plant-Made Therapeutics against Intestinal Inflammation and Colon Cancer" Owensboro Cancer Research Program. October 2014, Owensboro, $\mathrm{KY}$

5. Royal J, Baldauf K, Matoba N. "A Plant-Made Cholera Toxin B Subunit Variant Enhances Colonic Mucosal Wound Healing" University of Louisville Department of Pharmacology and Toxicology William J. Waddell Seminar Series 2017, March 2017, Louisville, KY 


\section{PATENTS}

\section{Applications}

1. Application Date: Jun 29, 2016

Application Number: PCT/US2016/040041

Title: Compositions and methods for treating cancer and promoting wound healing. Inventors: Nobuyuki Matoba, Keegan Baldauf, Joshua Royal

\section{ABSTRACTS AND PRESENTATIONS}

Oral Presentations: National/International Meetings

1. Royal J, Baldauf K, Kouokam J, Matoba N. "Plant-made cholera toxin B subunit as a candidate oral immunotherapeutic agent against ulcerative colitis" Plant-Based Vaccines, Antibodies \& Biologics. June 8 - 10, 2015, Lausanne, Switzerland.

2. Royal J, Oh Y, Galandiuk S, Matoba N. "Epicertin, A Cholera Toxin B Subunit variant, Enhances Intestinal Would Healing in a Mouse Acute Colitis Model and Human Ulcerative Colitis Colon Explants via an Unfolded Protein Response" Digestive Disease Week 2018, June 2 - 5, 2018, Washington, DC.

Oral Presentations: Local/Regional Meetings

3. Royal J, Baldauf K, Matoba N. "Plant-Made Cholera Toxin B Subunit: A Candidate Oral Immunotherapeutic Agent Enhances Colonic Mucosal Wound Healing" Center for Predictive Medicine Retreat, November 2016, Henryville, IN.

Poster Presentations: National/International Meetings

1. Royal J, Baldauf K, Yaddanapudi K, Dryden GW, Matoba N. "Oral Administration of a Cholera Vaccine Antigen Facilitates Colonic Mucosal Healing in a Murine Model of Colitis” Digestive Disease Week 2017, Chicago, IL, May 6 - 9, 2017.

2. Royal J, Youngjun Oh, Galandiuk S, Matoba N. "Epicertin Enhances Intestinal Wound Healing In A Mouse Colitis Model And Human Ulcerative Colitis Colon Explants" Crohn's and Colitis Congress, 2018, Las Vegas, NV

\section{Poster Presentations: Local/Regional Meetings}

1. Royal J, Nelson B, Baldauf K, Kouokam C, Matoba N. "Plant-Made Cholera Toxin B Subunit, an Orally Active Anti-inflammatory Protein, in an Acute Colitis Mouse Model: Investigation of Effective Dose and Time of Administration" Research Louisville 2014, Louisville, KY

2. Royal J, Husk A, Hamorsky K, Bennet L, Matoba N. "Plant-Made Anti-TNFa Monoclonal Antibody; an Infliximab Biosimilar against Intestinal Inflammation and 
Colon Cancer" James Graham brown Cancer center 14th Annual Retreat, 2014, Louisville, KY

3. Royal J, Baldauf K, Matoba N. "Plant-Made Cholera Toxin B Subunit: A Candidate Oral Immunotherapeutic Agent Enhances Colonic Mucosal Wound Healing" Research Louisville 2015, Louisville, KY

4. Baldauf K, Royal J*, Kouokam J, Dryden G, Matoba N. "Cholera toxin B subunit protects against colitis-associated colon cancer in a mouse model" Research Louisville 2015. October 28, 2015, Louisville, KY.

5. Royal J, Baldauf K, Matoba N. "Plant-Made Cholera Toxin B Subunit: A Candidate Oral Immunotherapeutic Agent Enhances Colonic Mucosal Wound Healing" Research Louisville 2016, Louisville, KY

6. Royal J, Oh Y, Matoba N. "Epicertin, A Cholera Toxin B Subunit Variant, Enhances Intestinal Wound Healing in a mouse acute colitis model and human ulcerative colitis colon explants" Research Louisville, 2017, Louisville, KY

\section{PUBLICATIONS}

\section{$\underline{\text { Peer-Reviewed Publications }}$}

1. Royal JM, Reeves MA, Matoba N. (2019) Repeated oral administration of a KDELtagged recombinant Cholera Toxin B subunit effectively mitigates DSS colitis despite a robust immunogenic response. Toxins 11(12). pii: E678.

2. Royal JM*, Oh YJ*, Grey MJ, Lencer WI, Ronquillo N, Galandiuk S, Matoba N. (2019) A modified cholera toxin B subunit containing an ER retention motif enhances colon epithelial repair via an unfolded protein response. FASEB J. 33(12): 1352713545 (PMID: 31560862 ) *Co-first authors

3. Royal JM, Matoba N. (2017) Therapeutic potential of Cholera Toxin B Subunit for the treatment of inflammatory diseases of the mucosa. Toxins. 9(12). pii: E379. (PMID: 29168738)

4. Baldauf KJ* and Royal JM*, Kouokam JC, Haribabu B, Jala VR, Yaddanapudi K, Hamorsky KT, Dryden GW, Matoba N. (2017) Oral administration of a recombinant cholera toxin B subunit promotes mucosal healing in the colon. Mucosal Immunol 10: 887-900. (PMID: 27805617) *Co-first authors

5. Baldauf KJ, Royal JM, Hamorsky KT, Matoba N. (2015) Cholera toxin B: One subunit with many pharmaceutical applications. Toxins 7: 974-996. (PMID: 25802972)

$\underline{\text { Non-peer reviewed articles, Book chapters, Proceedings, Pamphlet or Bulletin }}$

1. Royal, JM, Baldauf, K., Yaddanapudi, K., Dryden, GW, \& Matoba, N. “Oral Administration of a Cholera Vaccine Antigen Facilitates Colonic Mucosal Healing in 
a Murine Model of Colitis" Gastroenterology, 152(5), S572-S573. doi:10.1016/S0016-5085(17)32072-3

2. Royal JM, Oh Y, Galandiuk S, Matoba N, "Epicertin Enhances Intestinal Wound Healing in a Mouse Colitis Model and Human Ulcerative Colitis Colon Explants" Gastroenterology, 154(1), S16-S17. DOI: 10.1093/ibd/izy019.036

3. Royal JM, Oh Y, Galandiuk S, Matoba N, "983 - Epicertin, a Cholera Toxin B Subunit Variant, Enhances Intestinal Wound Healing in a Mouse Acute Colitis Model and Human Ulcerative Colitis Colon Explants via an Unfolded Protein Response" Gastroenterology, Volume 154 , Issue 6, S-183. doi:10.1016/S0016-5085(18)310217 